\title{
The Role of Metabolic Remodeling in Macrophage Polarization and Its Effect on Skeletal Muscle Regeneration
}

\author{
Francesca De Santa, ${ }^{1, *}$ Laura Vitiello, ${ }^{2, *}$ Alessio Torcinaro, ${ }^{1,3}$ and Elisabetta Ferraro ${ }^{2}$
}

\begin{abstract}
Significance: Macrophages are crucial for tissue homeostasis. Based on their activation, they might display classical/M1 or alternative/M2 phenotypes. M1 macrophages produce pro-inflammatory cytokines, reactive oxygen species (ROS), and nitric oxide (NO). M2 macrophages upregulate arginase-1 and reduce NO and ROS levels; they also release anti-inflammatory cytokines, growth factors, and polyamines, thus promoting angiogenesis and tissue healing. Moreover, M1 and M2 display key metabolic differences; M1 polarization is characterized by an enhancement in glycolysis and in the pentose phosphate pathway (PPP) along with a decreased oxidative phosphorylation (OxPhos), whereas M2 are characterized by an efficient OxPhos and reduced PPP.

Recent Advances: The glutamine-related metabolism has been discovered as crucial for M2 polarization. Vice versa, flux discontinuities in the Krebs cycle are considered additional M1 features; they lead to increased levels of immunoresponsive gene 1 and itaconic acid, to isocitrate dehydrogenase 1-downregulation and to succinate, citrate, and isocitrate over-expression.

Critical Issues: A macrophage classification problem, particularly in vivo, originating from a gap in the knowledge of the several intermediate polarization statuses between the M1 and M2 extremes, characterizes this field. Moreover, the detailed features of metabolic reprogramming crucial for macrophage polarization are largely unknown; in particular, the role of $\beta$-oxidation is highly controversial.

Future Directions: Manipulating the metabolism to redirect macrophage polarization might be useful in various pathologies, including an efficient skeletal muscle regeneration. Unraveling the complexity pertaining to metabolic signatures that are specific for the different macrophage subsets is crucial for identifying new compounds that are able to trigger macrophage polarization and that might be used for therapeutical purposes. Antioxid. Redox Signal. 30, 1553-1598.
\end{abstract}

Keywords: metabolic reprogramming, macrophage polarization, cross-talk muscle-macrophages, skeletal muscle regeneration, rehabilitation, mitochondria

\section{Table of Contents}

I. Introduction $\quad 1554$

II. МФ: Origin and Fate $\quad 1554$

$\begin{array}{ll}\text { A. Origin } & 1554\end{array}$

B. Activation/polarization 1556

1. M1 polarization 1556

2. M2 polarization 1556

Reviewing Editors: Esther Barreiro, Gabor Csanyi, Axel Gödecke, Mark Hannink, Ralf Kinscherf, Andreas Møller, Vladimir B. Ritov, Sashwati Roy, and Antonio M. Russo

\footnotetext{
${ }^{1}$ Institute of Cell Biology and Neurobiology (IBCN), National Research Council (CNR), Rome, Italy.

${ }^{2}$ Laboratory of Pathophysiology of Cachexia and Metabolism of Skeletal Muscle, IRCCS San Raffaele Pisana, Rome, Italy.

${ }^{3}$ Department of Biology and Biotechnology "Charles Darwin," Sapienza University, Rome, Italy.

*These two authors contributed equally to this work.
} 
III. Transcriptional Regulation of Polarization 1558

IV. Metabolic Features of M1 and M2 1561

A. M1-polarized MФ 1561

1. Enhanced glycolysis and reduced oxidative phosphorylation 1561

2. Pentose phosphate pathway 1562

3. Breakpoints in the TCA cycle and TCA intermediates accumulation 1562

\begin{tabular}{lr} 
a. Citrate/isocitrate level & 1562 \\
\hline b. Succinate & 1563
\end{tabular}

b. Succinate level 1563

B. M2-polarized MФ 1565

1. Enhanced OxPhos

2. Reduced PPP

3. Free fatty acids $\beta$-oxidation and glycolysis requirement in differentially polarized M $\Phi$ is controversial 1565

4. Glutamine-related metabolism 1566

C. NO and ROS in M1- and M2-polarized MФ 1567

1. NO/iNOS and ROS in M1 1567

2. NO/Arg1 and ROS in M2 1568

3. Redox signaling in M1 and M2 1568

V. Manipulating the Metabolism to Polarize MФ 1570

VI. M $\Phi$ and Skeletal Muscle Regeneration 1571

A. Skeletal muscle regeneration 1571

B. Role of MФ in skeletal muscle regeneration 1572

1. On acute injury 1572

a. M1-to-M2 switch in muscle regeneration 1574

2. In chronic muscle diseases: for example, Duchenne muscular dystrophy 1576

C. Cross-talk between metabolically diverse MФ subpopulations and muscle resident cells 1576

1. Cross-talk MФ: SCs 1577

2. Cross-talk MФ: FAPs 1579

VII. Metabolic Reprogramming of MФ as a Potential Therapeutical Approach to Improve Skeletal Muscle 1580

Regeneration

A. Aging

B. Duchenne muscular dystrophy 1580

C. Regenerative medicine 1580

D. Obesity-insulin-resistance 1580

E. Cachexia

F. Stimuli leading to metabolic reprogramming-mediated MФ polarization 1581

1. Exercise 1581

2. Calorie restriction 1582

3. Nutrients 1582

VIII. Conclusions 1582

\section{Introduction}

M

ACROPHAGES (MФ) ARE INFLAMMATORY CELLS with a high capacity for engulfing and digesting pathogens and cell debris. In addition, $\mathrm{M} \Phi$ play increasingly defined roles in orchestrating the healing of various damaged tissues and show high heterogeneity, plasticity, and adaptation abilities. Based on their environment and on the activation of specific signaling pathways, they might display, schematically, a pro-inflammatory (M1) or an anti-inflammatory (M2) phenotype, which will hereby be described. Differentially activated $M \Phi$ also show diverse metabolic features, with a link between the metabolic pathways operating in $М \Phi$ and their pro-/anti-inflammatory status; this review will focus on the potentiality of manipulating metabolism to polarize $\mathrm{M} \Phi$.

Among the various tissues in which $М \Phi$ play a role, their effect on skeletal muscle will be emphasized; differentially activated $\mathrm{M} \Phi$ are crucial for efficient muscle regeneration by means of a complex cross-talk with skeletal muscle resident cells. Specifically, MФ polarization can be metabolically modulated to improve the regenerative process; this aspect will be highlighted, with special focus on therapeutical approaches.

\section{II. МФ: Origin and Fate}

MФ represent one of the body's first lines of defense against pathogens. First described in 1887 by Metchnikoff as a population of phagocytes with the capacity to engulf and kill pathogens, $M \Phi$ are long-living cells of the innate immune system that also play a central role in adaptive immunity (156). They are present in all organs and tissues and can assume different phenotypes and functions; their diversity depends both on their origin and on the tissue and extracellular mileu in which they are located.

\section{A. Origin}

Most tissue МФ derive from circulating monocytes extravasating from the bloodstream into injured tissues where 
they differentiate into $M \Phi(8) . M \Phi$ are characterized by the expression of markers, including CD45, the colonystimulating factor 1 (CSF1)-receptor (CSF1R), CD11b, and the GPCR F4/80, none of which are uniquely restricted to MФ. Depending on the tissue, MФ can express different markers associated to their function in that tissue and can also assume different names (Fig. 1).

The study of $M \Phi$ ontogeny in mice showed that monocytes and $М \Phi$ derive from $М \Phi$ and dendritic cell progenitors (MDPs), which are bone marrow resident hematopoietic stem cells (c-kit ${ }^{+} \mathrm{CX} 3 \mathrm{CR} 1^{+} \mathrm{Flt} 3^{+} \mathrm{CD} 115^{+}$) (95). MDPs differentiate into a common monocyte precursor (c-kit ${ }^{+} \mathrm{CX} 3 \mathrm{CR} 1^{+} \mathrm{Flt} 3^{-} \mathrm{CD} 115^{+}$), which gives rise to the two main subsets of circulating monocytes, distinguished, in mice, by the expression of Ly6C and the chemokine receptor $\mathrm{CX} 3 \mathrm{CR} 1$; $\mathrm{Ly} 6 \mathrm{C}^{+} \mathrm{CX} 3 \mathrm{CR} 1^{\text {low }}$ are considered pro-inflammatory monocytes homing to inflamed tissues, whereas Ly6C ${ }^{\text {low }} \mathrm{CX} 3 \mathrm{CR} 1^{\text {high }}$ monocytes are less abundant and home to noninflamed healthy tissues contributing to tissue repair (102).
Most MФ derive from circulating monocytes, whereas some tissue-resident $\mathrm{M} \Phi$ arise from a distinct mechanism of hematopoiesis. For example, microglia is ontogenetically distinct from bone marrow-derived $М \Phi$ (BMDM), as it derives from yolk sac mesenchymal progenitors, which early in mouse embryo development migrate to the neuroepithelium without passing through a monocytic stage (105). Indeed, during mammal embryogenesis, hematopoiesis takes place in different organs (mainly yolk sac and fetal liver) before the hematopoietic stem cells establish in the bone marrow.

The first hematopoietic cells arising in mice are mesenchymal progenitors appearing at E7.5 in the yolk sac. These precursors generate erythrocytes and M $\Phi$ but not lymphocytes. Around E8.5, progenitors from the yolk sac begin to seed into the fetal liver and start the first wave of hematopoiesis. A second wave starts at E10.5 and takes place in the major arterial vessels of the aorta-gonad-mesonephros region. Clusters of hematopoietic precursors with endothelial features, once they are passed through different stages, give

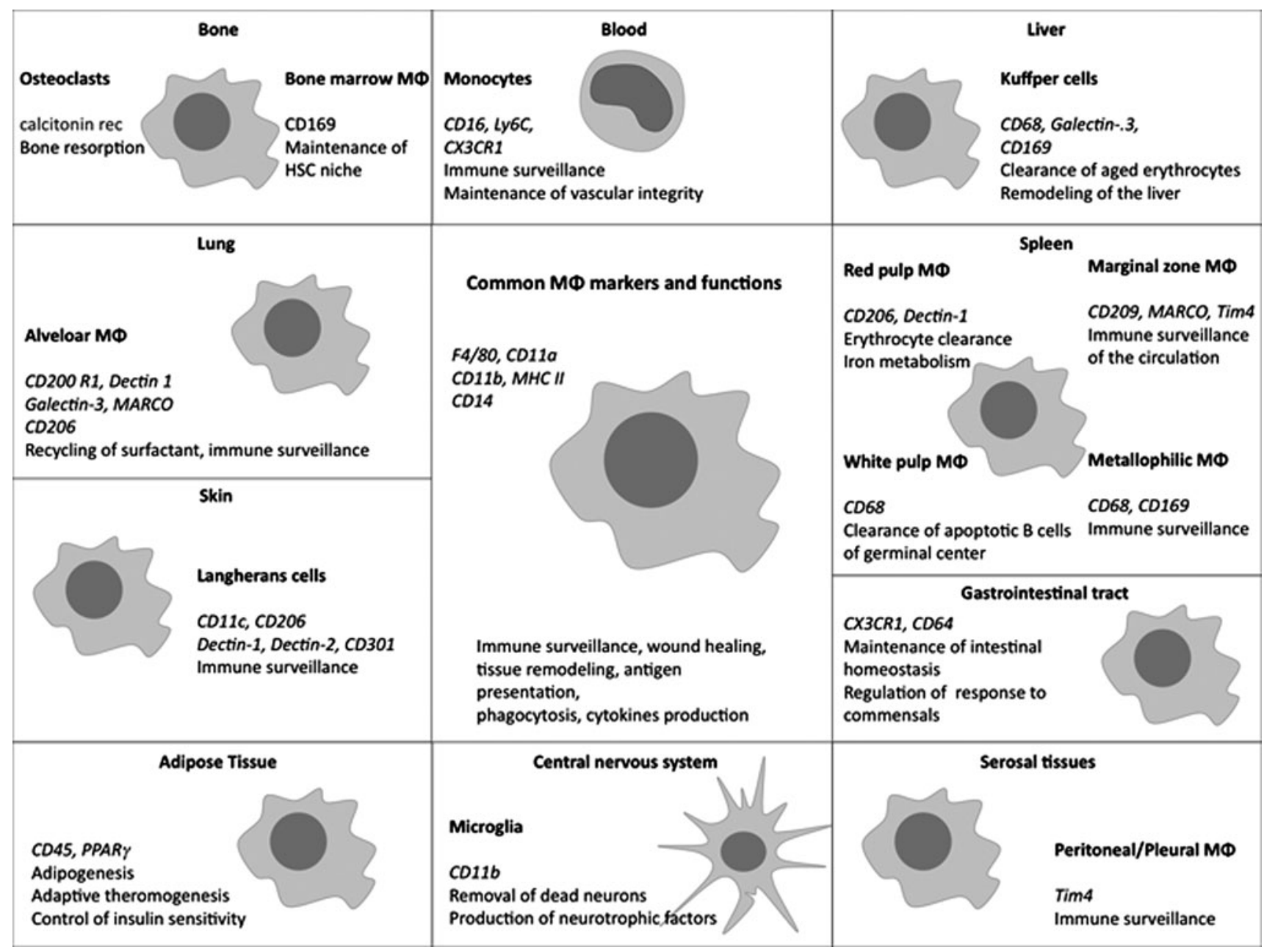

FIG. 1. Different phenotypes and specialized functions of $\mathbf{M \Phi}$ in different organs and tissues. Graphical schematization of tissue-specific МФ. МФ are spread throughout the body, in all tissues and organs. MФ in different compartments share some common features, expression markers, and functions (indicated in central panel). The perimeter panels describe tissue-specific $М \Phi$ with different names specific for each organ; in some cases, as in bone and spleen, different types of $M \Phi$ can be found in the same organ. For each $M \Phi$ type, a list of specific expression markers (surface markers or transcription factors) is provided, together with a summary of the main organ-specific functions. $М \Phi$ diversity is also highlighted by tissue-specific names: osteoclasts in the bones, Kupffer cells in the liver, microglia in the brain, alveolar $M \Phi$ in the lung, marginal-zone and red-pulp MФ in the spleen, and subcapsular sinus and medullary MФ in lymph nodes. MФ, macrophages. 
rise to the definitive hematopoietic stem cells that are able to differentiate into multiple lineages (336). After E16.5, the transition to the bone marrow-the definitive site of hematopoiesis - takes place.

Besides microglia, long-lived embryonic precursors persist during adulthood and contribute to other resident $\mathrm{M} \Phi$ in murine adult tissues - such as Kupffer cells in the liver or alveolar M $\Phi$ in the lung-whereas $\mathrm{M} \Phi$ in other tissues are replaced. Thus, at least in mice, two ontogenetically distinct MФ populations persist in adults, the ones derived from the bone marrow and the ones derived from the yolk sac. The latter population proliferate and sustain their presence in vivo independently of bone marrow-derived monocytes. Indirect evidence suggests that also in humans, some tissue $М \Phi$ are long-lived cells existing independently of circulating monocytes (269).

\section{B. Activation/polarization}

MФ are phagocytes that respond to danger signals, sensing injury and infection; activated $\mathrm{M} \Phi$ engulf and digest invading and damaged cells. They also present the antigen to lymphocytes and produce cytokines, thus further enhancing inflammation. In addition, $\mathrm{M} \Phi$ are crucial for tissue homeostasis $(232,312)$; indeed, depending on their activation and on their microenvironment, $М \Phi$ might play proinflammatory or anti-inflammatory roles, thus leading to a gross classification into two groups: M1 and M2 (198, 213).

M1 are obtained by "classical" activation; they remove foreign and damaged cells thanks to their highly bactericidal and phagocytic capacity. Conversely, M2, obtained by "alternative" activation, resolve inflammation and mediate tissue regeneration and angiogenesis. $М \Phi$ are plastic cells that readily switch their phenotype. With a wide-ranging agreement among scientists, the acquisition of different phenotypes by $\mathrm{M} \Phi$ is referred to as polarization $(189,196,234)$.

1. M1 polarization. M1 polarization is elicited by interferon- $\gamma(\mathrm{IFN} \gamma)$ priming associated to pro-inflammatory cytokines, for example, tumor necrosis factor- $\alpha(\mathrm{TNF} \alpha)$, or by Toll-like receptor (TLR) ligands such as microbial products like pathogen-associated molecular patterns (PAMPs) or opsonins. PAMPs are molecules binding to TLRs that are highly conserved in different classes of pathogens, for example, lipopolysaccharide (LPS) - a component of the Gram-negative bacterial membrane-binding TLR4. LPS is commonly used to induce M1 activation in vitro. Other PAMPs are flagellin, peptidoglycans, and viral doublestranded RNA.

The activation of TLRs, together with NOD-like receptors, by PAMPs as well as by danger-associated molecular patterns (DAMPs) from the damaged tissue or by alarmins leads to M1 polarization. DAMPs are small molecules that are highly concentrated within healthy cells but absent or rare in the extracellular matrix (ECM), such as the high mobility group box 1 (HMGB1) protein regulating chromatin organization in healthy cells, nucleotides (ATP, ADP, UDP), oxidized phospholipids, heat shock proteins, and uric acid (224).

DAMPs are rapidly released after unprogrammed cell death, and they bind pattern recognition receptors (PRRs) on the immune cells' surface that become activated and start the inflammatory response. PRRs binding DAMPs might either be the same as those binding PAMPs (e.g., TLR) or be unique for DAMPs, such as RAGE (receptor for advanced glycation end products) binding HMGB1 or purinergic (P2) receptors sensing extracellular nucleotides, such as P2X7. МФ activation by DAMPs occurs in case of sterile inflammation, meaning in the absence of pathogens like in muscle injury, where cellular debris triggers M1 polarization.

Differentially activated $M \Phi$ subsets might be identified by the spectrum of secreted soluble factors. Briefly, M1 produce antiviral proteins, pro-inflammatory cytokines, and chemokines (Fig. 2) [for review see Mortha and Burrows (223)]; high amounts of reactive oxygen species (ROS) and nitric oxide (NO), the latter mainly by inducible NO synthase (iNOS), which is considered among the most reliable markers of M1 activation (see section IV.C) (224, 280). M1 are characterized by an increased killing rate and antigen presentation ability (224). In addition, some cell surface molecules highly expressed in M1 compared with unstimulated MФ (M0) or M2 are used as M1 polarization markers (Fig. 2) (373).

However, the high degree of $M \Phi$ plasticity and their ability to switch from a phenotype to another, through intermediate polarization status, makes the identification of unambiguous membrane markers distinguishing M1 from M2 difficult. Indeed, differently polarized $M \Phi$ can be better identified by also considering the pattern of cytokines and chemokines secreted, the effect exerted on surrounding tissues, and their metabolic status (see section IV) (313).

2. M2 polarization. On the other hand, M2 phenotypical activation is favored in normal tissue homeostasis and during recovery of tissues after damage, where M2 are necessary to resolve the inflammatory response and to allow tissue healing and remodeling. Anti-inflammatory and regenerative M2 produce interleukin (IL)-10, IL-1ra (IL-1 antagonist), growth factors, and polyamines (Fig. 2), which stimulate fibroblast growth, collagen, and production of ECM components, thus promoting fibrosis, angiogenesis, lymphangiogenesis, and damaged tissue healing.

M2 also play a role in parasite infection and tumor progression $(114,382)$. ROS production is reduced in M2 and arginase-1 (Arg1) is upregulated, thus also lowering NO production. By secreting IL-10, which inhibits T helper (Th)1 lymphocytes and inflammatory cytokine production, and by recruiting regulatory $\mathrm{T}$ cells in damaged sites, $\mathrm{M} 2$ directly contribute to dampen inflammation; in fact, inflammation is necessary for protection against infections, but if prolonged, it might cause tissue damage (148).

M2 activation is fostered by anti-inflammatory, protolerogenic Th2 cytokines such as IL-10, IL-4, and IL-13; whereas it might be inhibited in a context in which Th1/Th17 cytokines dominate. Macrophage colony-stimulating factor (M-CSF), systemically expressed and crucial for МФ differentiation from precursor cells, can also prime and support M2 polarization (although not acting by itself) by inducing the expression of M2-associated genes (Fig. 2) together with cell cycle regulatory genes (cyclins) that foster homeostatic proliferation $(199,287)$. The broad availability of M-CSF contributes to maintaining the pool of tissue resident M2 (67).

M2 upregulate Arg1 and activate the arginine pathways, producing ornithine and polyamines (see section IV.C.2). As $\mathrm{NO}$ and ornithine are directly involved in pathogen killing, 
M1

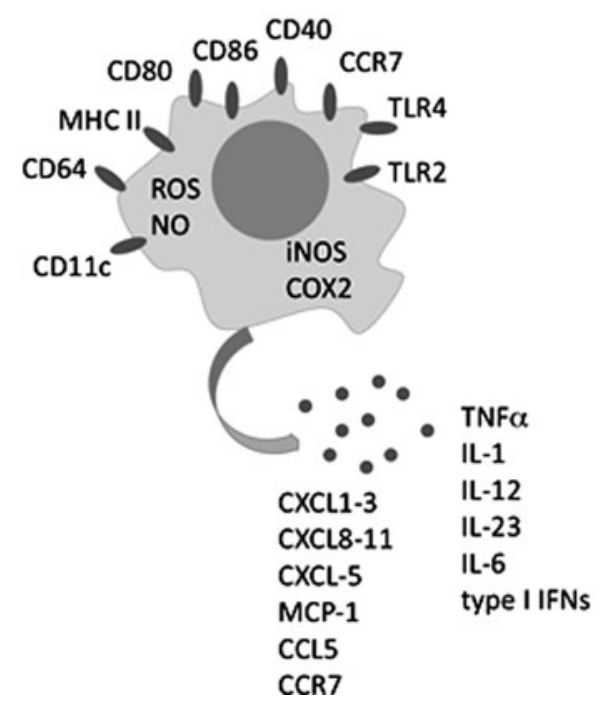

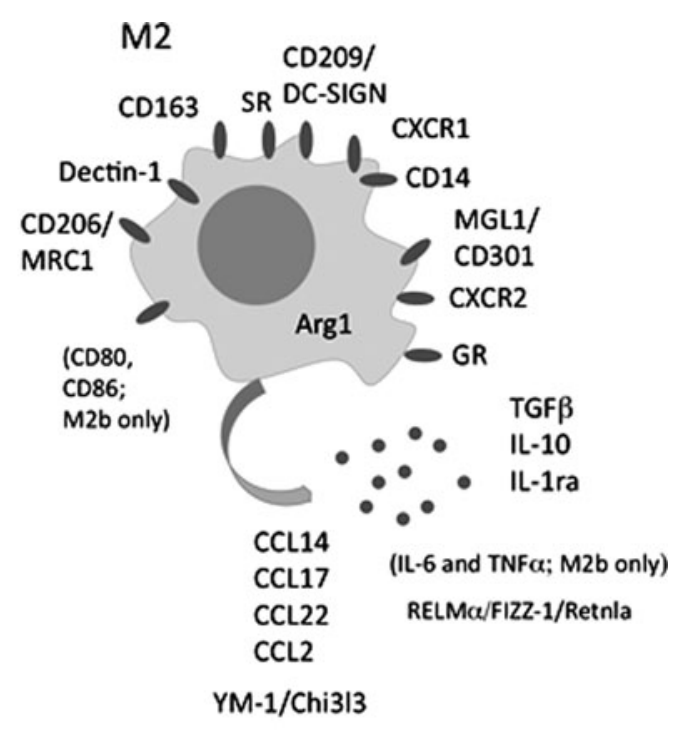

FIG. 2. M1 and M2 markers. On specific stimulation, $M \Phi$ can assume different phenotypes and exert different functions. The M1 and M2 phenotypes represent the two extremes of intermediate states displaying mixed functions and markers. This graphical schematization depicts the principal markers, including membrane markers and released factors, used to identify the polarization status of M1 and M2. Some markers expressed only by the M2b subtype are indicated in brackets. M1 M $\Phi$ produce pro-inflammatory cytokines and chemokines such as TNF $\alpha$, IL-1, IL-18, IL-6, IL-12, IL-23, type I IFNs (IFN $\alpha$ and IFN $\beta$ ), MCP1/CCL2, CXCL5, and CXCL8. The type II IFN $\gamma$, produced by T lymphocytes and NK cells, promotes, along with TLR ligands (or with CD40L expressed by T lymphocytes), a further M1 polarization via the IFN $\gamma$ receptor. Cell surface molecules are expressed at high levels in M1, for example, CD80, CD86, MHC-II, TLR2, TLR4, CD64, and CD40. M2 M $\Phi$ produce IL-10, IL-1ra, growth factors such as IGF-1, PDGF, TGF- $\beta$, VEGF, FGF, and polyamines. M2-associated genes are Arg1, MRC1/CD206, IL-4R, RELM $\alpha /$ Fizz1/Retnla, and Chi313/YM-1. Cell surface molecules over-expressed in M2 and used as M2-specific markers are Dectin-1, DC-SIGN, mannose, and galactose-type receptors, for example, CD206, scavenger receptor A, scavenger receptor B1, CD163, MGL1-2/CLEC10A/CD301/Lectin, MARCO, CXCR1, and CXCR. Several other molecules have been proposed as M $\Phi$ markers, including CD200R, transglutaminase-2, and CD23, as reviewed by Roszer (287). Arg1, arginase-1; Chi313, chitinase 3-like 3; CXCL, CXC chemokine ligand; DC-SIGN, dendritic cell-specific ICAM-grabbing nonintegrin; FGF, fibroblast growth factor; IFN, interferon; IGF-1, insulin-like growth factor-1; IL, interleukin; MARCO, МФ receptor with collagenous structure; MCP1, monocyte chemoattractant protein 1; MGL1-2/CLEC10A, MP galactose-type calcium-type lectin/C-type lectin domain family10, member A; MHC-II, major histocompatibility complex II; MRC1, mannose receptor C type 1; PDGF, plateletderived growth factor; RELM $\alpha /$ Fizz1, resistin-like molecule $\alpha /$ found in inflammatory zone 1 ; TGF- $\beta$, transforming growth factor- $\beta$; TLR, Toll-like receptor; TNF $\alpha$, tumor necrosis factor- $\alpha$; VEGF, vascular endothelial growth factor.

angiogenesis, and tissue repair, these molecular signatures are considered the most typical features of M2 activation (212). Cell surface molecules over-expressed in M2 and used as their markers are indicated in Figure 2 and reviewed by Rőszer $(198,287)$. Notably, many of the reported M1 and M2 markers are not specific for $\mathrm{M} \Phi$ but are also expressed by other cell types.

M2 have a high degree of heterogeneity and a further classification into three subgroups-M2a, M2b, and M2c-has been proposed, mainly on the basis of the inducing stimuli and on the panel of secreted factors (195). All three M2 subtypes are characterized by high IL-10 and low IL-12 levels.

M2a - where "a" stands for "alternative" - are induced by IL-4/IL-13 stimulation; they produce high levels of IL-10 and IL-1ra and express the mannose receptor CD206 and the receptor IL-1RII, a nonsignaling molecule that acts as a decoy receptor for IL-1. M2a are involved in Th2 responses, allergy, and the killing and encapsulation of parasites (195).

$\mathrm{M} 2 \mathrm{~b}$ are induced by exposure to immunocomplexes and agonists of IL-1R or TLRs such as LPS, thus displaying a proinflammatory cytokine profile, as they produce, together with IL-10, discrete amounts of IL- 6 and TNF $\alpha$ but a very low amount of IL-12. As discussed later, similar to M1, M2b do not express high levels of $\operatorname{Arg} 1$, which is a marker for M2a and M2c (195).

M2c are induced by IL-10 and glucocorticoid hormones and are also referred to as "deactivated" MФ. M2c express CD206, produce Arg1, and secrete IL-1ra, IL-10, and transforming growth factor- $\beta$ (TGF- $\beta$ ). Moreover, they also express CD163, which is not expressed by other M2 subtypes (158). M2c are involved in tissue remodeling, ECM deposition, and immunoregulation (195). Interestingly, under the effect of IL-10, they express discrete amounts of CCR2 and CCR5 receptors for pro-inflammatory chemokines (MCP1/ CCL2, RANTES/CCL5, macrophage inflammatory protein (MIP) $1 \alpha / C C L 3$, and MIP1 $\beta /$ CCL4), which, in a mileu rich in IL-10, would serve as a scavenger receptor system to dampen inflammation (270).

Additional $\mathrm{M} \Phi$ phenotypes have been more recently identified; some of them-namely Mox, $\mathrm{M}(\mathrm{Hb})$, Mhem, and M4-have been described in the atheresclerotic plaque, both in humans and in mice $(22,107,154)$. These phenotypes are characterized by specific gene expression profiles and are elicited by different stimuli, such as oxidative stress 
(oxidized phospholipids) for Mox, hemoglobin-haptoglobin complexes for $\mathrm{M}(\mathrm{Hb})$, heme for Mhem, and $\mathrm{CXC}$ chemokine ligand (CXCL)4 for M4. Mox M $\Phi$ are believed to have a particular ability to deal with oxidative stress, as they express an increased glutathione/oxidized glutathione (GSH/GSSG) ratio (154) and the generation of the Mox phenotype is mediated by nuclear factor (erythroid-derived 2)-like 2 (Nrf2).

The various origins of $M \Phi$ (from circulating monocytes or from yolk sac), their polarization to pro-inflammatory or antiinflammatory subsets, and their heterogeneity have been described. The nomenclature M1 and M2 emulates the T cells Th1/Th2 classification and underlines the functional cross-talk between lymphocytes and MФ: Th1 lymphocytes produce $I N F \gamma$, required for M1 polarization, and Th2 secrete IL-4 and IL-13, which drive M2 polarization. Notably, this classification is simplistic, due to the occurrence of several MФ intermediate polarization statuses (234). The obstacles and lack of consensus in defining МФ activation are described by the guidelines of Murray and coauthors (234). Based on such guidelines, describing $\mathrm{M} \Phi$-activation in vivo requires an explicit description of the populations under investigation, how they were isolated, from which tissue and conditions, and which marker combinations were used to ascertain $\mathrm{M} \Phi$ activation.

\section{Transcriptional Regulation of Polarization}

A number of transcriptional factors participate in the differential activation of $M \Phi$. In this section, we describe the most relevant, including the signal transducers and activators of transcription (STATs), the IFN-regulatory factors (IRFs), the nuclear factor $\kappa \mathrm{B}(\mathrm{NF}-\kappa \mathrm{B})$, the hypoxia-inducible factors (HIFs), Krüppel-like factors (KLF), the peroxisome proliferator-activated receptors (PPARs), and several microRNAs (miRNAs) $(170,315)$.

The members of the STAT family involved in MФ polarization are STAT1, STAT2, STAT3, and STAT6. During M1 polarization, IFN $\gamma$ binds to its receptor and triggers Janus kinase 1/2 (JAK1 and JAK2)-mediated phosphorylation of STAT1, which dimerizes and binds to the promoter region of target genes, including $i N O S$ and $I L-12$ (Fig. 3) (66). STAT1 and STAT2 are also involved in the autocrine response to IFN $\beta$, released by LPS-activated $\mathrm{M} \Phi$; IFN $\beta$ triggers the formation of the STAT1-STAT2 heterodimers that bind to IRF9 (Fig. 3) (see below within this same paragraph). In STAT1-knockout (KO) mice, MФ fail to produce TNF $\alpha$ and NO, thus demonstrating that STAT1 is required for M1 polarization (179).

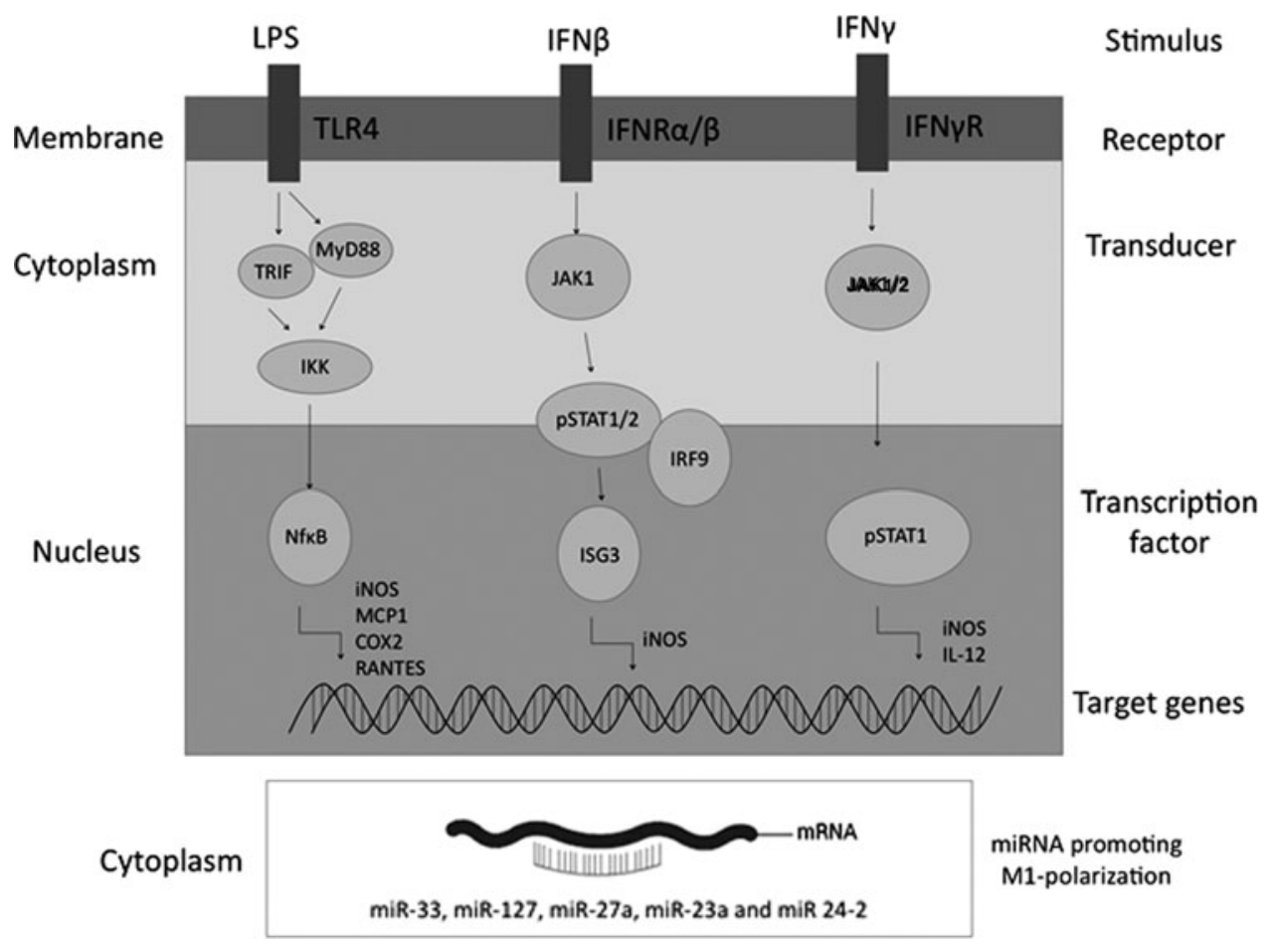

FIG. 3. Main pathways in M1. The main regulatory pathways in M1 polarization are represented here. M1 polarization is fostered by pro-inflammatory stimuli such as LPS and IFNs. LPS binds to TLR4, which, through MyD88-dependent and MyD88-independent (TRIF-dependent) pathways, induces the activation of IKK. IKK, in turn, phosphorylates IkB, which is degraded, thereby releasing NF- $\kappa \mathrm{B}$. NF- $\kappa \mathrm{B}$ migrates to the nucleus and drives the transcription of several genes that have the NF- $\kappa \mathrm{B}$ binding sites in their promoter region, for example, iNOS, MCP1, COX-2, and RANTES. IFN $\beta$ binds to the IFN $\alpha / \beta$ receptor that activates JAK3. JAK3 phosphorylates STAT1 and STAT2 that bind IRF9 to form the ISGF-3, which drives the transcription of iNOS. IFN $\gamma$ binds to IFN $\gamma$ receptors, thus activating JAK1/2 that phosphorylate STAT1. pSTAT1 forms a homodimer that binds to DNA to induce the transcription of iNOS and IL-12. Some miRNAs are crucial for M1 polarization (e.g., miR-33, miR-127, miR-27a, miR-23a, and miR 24-2). COX-2, cyclooxygenase-2; IFN $\beta$, interferon- $\beta$; IKK, IkB kinase; iNOS, inducible NO synthase; IRF9, IFN-regulatory factor 9; ISGF-3, interferon-stimulated gene factor-3; JAK3, Janus kinase 3; LPS, lipopolysaccharide; MCP1, monocyte chemoattractant protein 1; miRNAs, microRNAs; MyD88, myeloid differentiation primary response gene 88 ; NF- $\kappa \mathrm{B}$, nuclear factor $\kappa \mathrm{B}$; NO, nitric oxide; RANTES, regulated on activation, normal T cell expressed and secreted; STATs, signal transducers and activators of transcription; TLR4, Toll-like receptor 4; TRIF, TIR-domain-containing adapter-inducing interferon- $\beta$. 
Conversely, STAT2 deficiency in mice does not block M1 polarization completely, but it impairs autocrine response to type I IFNs (309). STAT3 is considered one of the key signaling molecules in the induction of the M2 phenotype; STAT3 is activated by IL-10 and, in turn, enhances $I L-10$ expression while inhibiting TNF $\alpha$, IL- $1 \beta$, and IL-12 production. Notably, STAT3 activation by IL-6-considered a prototypic inflammatory cytokine-in a pro-M2 mileu (i.e., in the presence of IL-4/IL-13) enhances M2 polarization (90). Stimulation of M $\Phi$ by IL-4/IL-13, both binding the IL-4 receptor- $\alpha$ (IL-4R $\alpha$ ) and activating either JAKe1/JAK3 (by IL-4) or JAK1/Tyk2 (by IL-13), induces the phosphorylation and activation of STAT6, which dimerizes and induces the expression of most M2 genes, for example, Arg1, CD206, resistin-like molecule alpha $($ RELM $\alpha) / F i z z 1 / R e t n l a$, and Yml (Fig. 4).

IRFs are also implicated in MФ polarization. IRF1 (induced by type I IFNs) and IRF5 (induced by TLR) are required for proper M1 polarization and secretion of pro-inflammatory cytokines $(327,384)$; indeed, IRF5 polymorphisms are associated with immune-mediated diseases (115). IRF3 and IRF7 are activated in M1 and trigger type I IFNs production. IRF9 binds to the STAT1/STAT2 heterodimer to form the IFNstimulated genes (ISG) factor 3, which stimulates the transcription of several M1-related genes such as $i N O S$ (Fig. 3). On the other hand, IRF4 is specifically associated with M2 activation, as it is induced by IL-4-mediated activation of STAT6.

LPS binding to TLR4 and CD14 during M1 polarization fosters NF- $\kappa \mathrm{B}$ activation, through myeloid differentiation primary response gene 88 (MyD88)-dependent and MyD88independent but TIR-domain-containing adapter-inducing interferon- $\beta$ (TRIF)-dependent pathways. NF- $\kappa \mathrm{B}$ is normally present-but kept inactive by $\mathrm{IkB}$ - in the $\mathrm{M} \Phi$ cytoplasm. TLR4 stimulation activates IkB kinase, which phosphorylates IkB and targets it for degradation; NF- $\kappa \mathrm{B}$ is, therefore, released and migrates to the nucleus, where it contributes to its own activation by upregulating $I L-1 \beta$ and $T N F \alpha$, these, in turn, stimulating $\mathrm{NF}-\kappa \mathrm{B}$ activation.

Other genes with NF- $\kappa \mathrm{B}$-binding sites in their promoters are $i N O S$, monocyte chemoattractant protein 1 (MCP1)/ $C C L 2$, cyclooxygenase-2 (COX-2), and regulated on activation, normal $\mathrm{T}$ cell expressed and secreted (RANTES)-CCL5, all related to $\mathrm{M} 1$ polarization. However, also $\mathrm{M} 2$ activation is partly regulated by NF- $\kappa \mathrm{B}$; in fact, $\mathrm{KO}$ mice for the NF- $\kappa \mathrm{B}$ repressor, $p 50-N F-\kappa B$, display not only an exacerbated M1 response but also an impaired ability to establish a proper M2 response during parasitic infections (276). Also, the transcription factor activator protein 1 (AP1) is activated by TLRs.

A condition of hypoxia, occurring in damaged tissues and in case of bacterial infection-also due to high oxygen

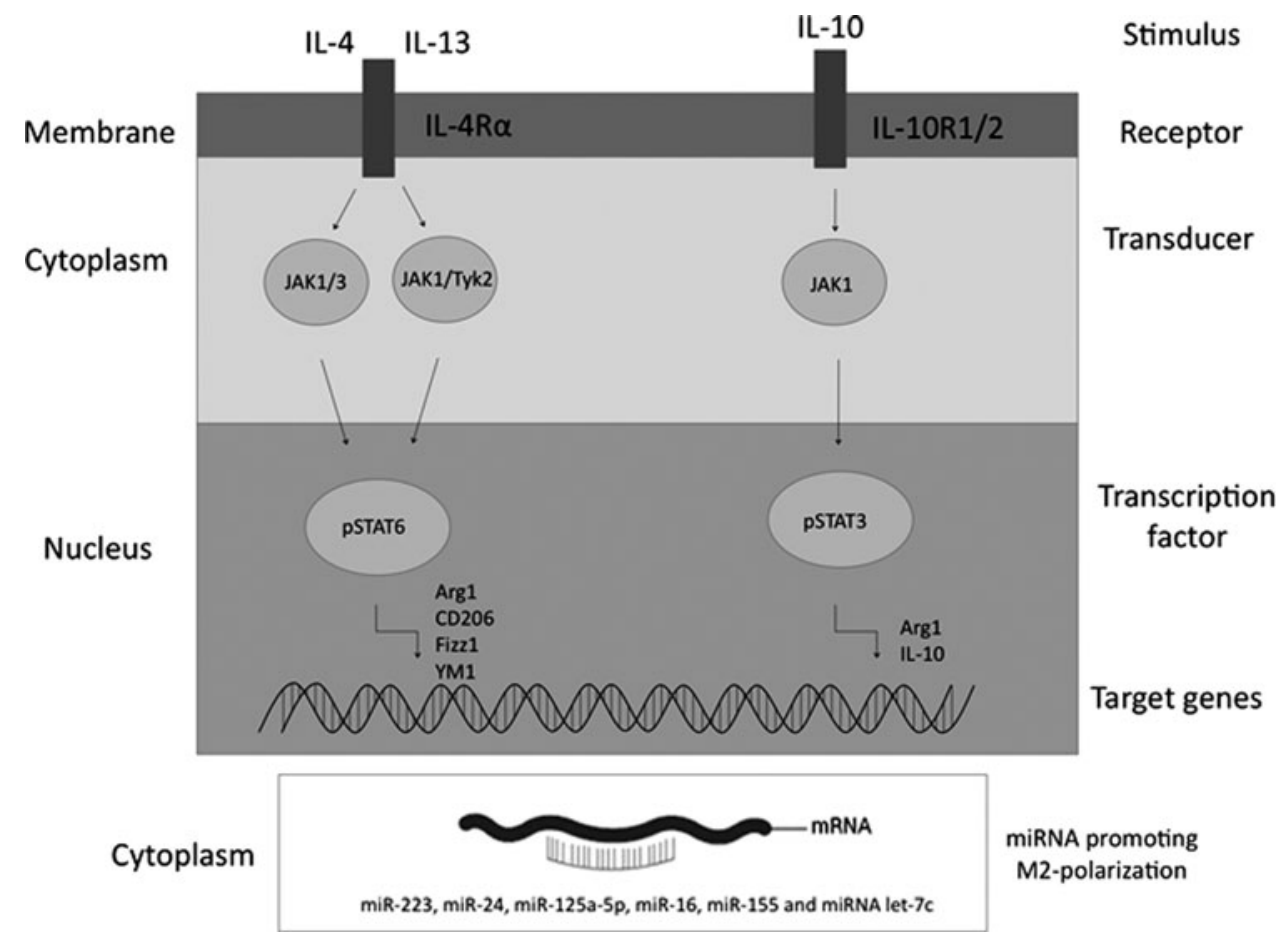

FIG. 4. Main pathways in M2. The main regulatory pathways in M2 polarization are represented here. M2 polarization is induced by pro-tolerogenic cytokines, namely IL-4, -13, and -10 that activate the JAK/STAT pathway. IL-4 and IL-13 act through the same receptor, the IL-4R $\alpha$. The engagement of the receptor leads to the formation of JAK1/JAK 3 or JAK1/Tyk2 dimers (when stimulated by IL-4 or by IL-13, respectively), fostering the phosphorylation of STAT6. pSTAT6 binds to DNA and induces the transcription of the mannose receptor (CD206), Arg1, Fizz1, and Ym1 genes. Phosphorylation of STAT3 and STAT6 induces the expression of M2-specific genes. IL-10 binds to the IL-10R1/2, a transmembrane receptor complex consisting of two different chains, IL-10R1 and IL-10R2. The signal is transduced through the activation of JAK1, which phosphorylates STAT3. pSTAT3 drives the transcription of Arg1 and of IL-10, thus creating an amplification loop. Some miRNAs, including miR-223, miR-24, miR-125a-5p, miR-16, miR-155, and miRNA let-7c, suppress the pro-inflammatory response, favoring an M2 phenotype. IL-4R $\alpha$, IL-4 receptor- $\alpha$; IL-10R1/2, IL-10 receptor $1 / 2$. 
consumption by inflammatory cells - can influence M $\Phi$ polarization. The effect of low oxygen partial pressure $\left(\mathrm{pO}_{2}\right)$ on cells is mediated by HIFs, two of which (HIF- $1 \alpha$ and HIF- $2 \alpha$ ) are also expressed by $\mathrm{M} \Phi(57,64)$. Low $\mathrm{pO}_{2}$ reduces the prolyl hydroxylases (PHDs)-dependent degradation of HIF- $1 \alpha$ and HIF- $2 \alpha$, which can dimerize with HIF- $1 \beta$ and become active gene regulators. HIF- $1 \alpha$ and HIF- $2 \alpha$ isoforms are directly involved in M1 and M2 polarization, due to their influence, in an antagonistic manner, on NO metabolism inducing the expression of $i N O S$ and Arg 1, respectively, although Arg 1 seems to be a target of both HIF isoforms $(28,328)$.

$H I F-1 \alpha$ plays a crucial role in orchestrating part of the M1 polarization, since it downregulates $C D 206$, enhances $I L-1 \beta$ and the expression of other pro-inflammatory genes (324), and stimulates glycolysis as well as pyruvate dehydrogenase kinase-1 (PDK1) and glucose transporter type-4 (GLUT4) expression to allow ATP production in hypoxia; indeed, $H I F$ $1 \alpha-\mathrm{KO}$ (but not $H I F-2 \alpha-\mathrm{KO}$ ) mice show alternative MФ polarization and wound-healing improvement. In M1, HIF-1 and NF- $\kappa \mathrm{B}$ are activated in parallel by hypoxia and ROS and act synergistically to induce common target genes such as $i N O S$; however, in some conditions, $\mathrm{NF}-\kappa \mathrm{B}$ seems to repress HIF-1 target genes, causing alternative polarization under hypoxia.

On the other hand, since NADPH-oxidases (NOX) and iNOS use oxygen to produce ROS or NO, a low $\mathrm{pO}_{2}$ might reduce their activity and $\mathrm{M} 1$ pro-inflammatory function. HIF$2 \alpha$ is barely detectable in M1, whereas it is upregulated in M2 where $H I F-1 \alpha$ is downregulated. However, a decrease of the pro-inflammatory response has also been detected in $H I F-2 \alpha$ $\mathrm{KO}$ mice [for references see Brüne et al. (28)]. Besides Arg1, HIF-2 triggers anti-oxidant protein expression and ECM production. In M2, both HIF isoforms promote vascular endothelial growth factor (VEGF) signaling and angiogenesis, which are crucial for the regenerative process.

Interestingly, it has been found that PHD2 downregulation is critical for alternative polarization and promotes capillarization and arteriogenesis independently of hypoxia through the NF- $\kappa$ B pathway (120); activation of the angiopoietin receptor TIE2 in ischemia-associated $\mathrm{M} \Phi$, triggered by endothelium shear stress-released VEGF, induces PHD2 downregulation in normoxia, which activates the canonical NF- $\kappa \mathrm{B}$ pathway, further upregulating the proarteriogenic TIE2. This is associated to M2 polarization and enhanced capillarization; indeed, arteriogenesis occurs in sites distant from the hypoxic area (120). It has more recently been observed that, in human MФ under hypoxic conditions, a transcriptomic switch triggered by both HIF- $1 \alpha$ and HIF- $2 \alpha$ together (not by HIF- $1 \alpha$ alone) occurs; this switch upregulates glycolytic enzymes, increasing anaerobic glycolysis (143).

HIF isoforms are differentially activated, HIF- $1 \alpha$ being induced by Th1 cytokines and HIF- $2 \alpha$ by Th 2 cytokines (e.g., IL-4). HIF- $1 \alpha$ clearly accumulates in M1 in normoxia conditions as well, paired with the contribution of $\mathrm{NF}-\kappa \mathrm{B}$ binding to its promoter. HIFs are also redox-sensitive transcription factors, since PHD is influenced not only by oxygen but also by NO and ROS. M1-produced ROS reduce PHD activity and promote HIF-1 $\alpha$ expression also under an ambient oxygen level. Also, NO represses PHD and stabilizes HIF-1, which, in turn, induces $i N O S$ in a positive feedback typical of M1 (see sections IV.C.1, IV.A.3.2, and V). However, NO becomes destabilizing under hypoxic conditions; by competing with oxygen for the binding to cytochrome $c$ oxidase (COX), NO might leave more oxygen available for PHD activity (28).

Finally, LPS-induced TLR4 signaling triggers a cross-talk between HIF- $1 \alpha$ and apoptosis signal-regulating kinase-1 (ASK1) pathways, both activated by ROS. ASK1 contributes to HIF- $1 \alpha$ stabilization likely via p38 mitogen-activated protein kinase (MAPK), which is typically involved in M1 polarization $(164,322,334)$. Even though HIF-1 $\alpha$ levels mostly depend on PHD, also nuclear factor of activated T cells (NFAT) and STAT3 - crucial for the M2 phenotypeseem to be required for $H I F-1 \alpha$ expression as well as for the angiogenic role of $\mathrm{M} \Phi$; whereas transcriptional regulation of $H I F-2 \alpha$ is barely known [for references see Brüne et al. (28)]. Through HIF-1 $\alpha$, hypoxia also upregulates some DAMP receptors such as $R A G E$ and $P 2 X 7 R$, thus influencing МФ polarization (325). HIFs promote stemness as well, including that of human embryonic stem cells, thus increasing their regenerative potential. Stem cells reside within hypoxic regions and HIFs are involved in their homeostasis also by decreasing their reliance on oxidative metabolism. HIFs maintain stemness also in cancer stem cells (203).

KLFs are zinc-finger transcriptional factors. Several members of the KLF family are involved in the MФ-polarization process. KLF2, KLF10, and KLF13 attenuate M1 polarization through different pathways: (i) KLF2 inhibits NF- $\kappa \mathrm{B}$ mediated response to pro-inflammatory cytokines; (ii) KLF10 binds the promoter of TGF- $\beta R I I$ (257); and (iii) KLF13 mediates the anti-inflammatory effect of miR-125a-5p (11). On the other hand, KLF6 enhances the expression of proinflammatory genes in MФ (159). As for KLF4, its function is controversial; it is upregulated in LPS/IFN $\gamma$-stimulated murine $\mathrm{J} 774 \mathrm{M} \Phi$, where it cooperates with $\mathrm{NF}-\kappa \mathrm{B}$ to enhance $i N O S$ expression (89); whereas by contrast, it is over-expressed in murine peritoneal M2 and adipose tissue M2 MФ from obese humans, where it interacts with STAT6 and inhibits NF- $\kappa \mathrm{B}-$ mediated M1 response (182).

$\operatorname{PPAR} \gamma$ is known to induce M2 activation; in fact, administration of rosiglitazone, a PPAR $\gamma$ agonist, leads to an increased expression of Arg1, found in inflammatory zone 1 (Fizz1), and IL-10 (127). On exposure to Th2 stimuli mediating M2 polarization, PPAR $\gamma$ deficiency downregulates Argl and IL-10 likely through the induction of miR-223 (392). Inhibition of pro-inflammatory cytokine signaling, for example, by suppressor of cytokine signaling (SOCS) proteins, also occurs in M2.

Several miRNAs have emerged as crucial players in MФ polarization (Figs. 3 and 4) (148, 243, 253, 330, 391, 392). Along with miRNAs, other epigenetic mechanisms modulate MP polarization; just to give some examples of an extremely wide field, methylation of lysine 4 of histone 3 (H3K4) seems to be involved in $T N F \alpha$ transcriptional regulation (181). On the other hand, Xia et al. reported that methylation of $\mathrm{H} 3 \mathrm{~K} 4$ leads to $T N F \alpha$ and $I L-6$ downregulation in a murine model of sepsis (383). Moreover, the histone demethylase Jumonji domain-containing 3 seems to not only contribute to a fine regulation of LPS-dependent M1 gene expression (68) but also be crucial for M2 polarization (303). Further, histone acetylation represents another mechanism of transcriptional control; for example, histone deacetylases 3 (HDAC3) removes the acetylation on the enhancers of IL-4-induced genes, thus representing a brake for M2 polarization (148, 230). 


\section{Metabolic Features of M1 and M2}

In addition to the earlier described features characterizing M1 and M2, these two subsets might also be distinguished by key metabolic differences. Several years ago, Newsholme and collaborators proposed that $\mathrm{M} \Phi$ polarization in mice is associated to a metabolic reprogramming; in synthesis, the switch toward a glycolytic or an oxidative metabolism is required for M1 and M2 polarization, respectively. Quiescent $\mathrm{M} \Phi$ rely mostly on mitochondrial oxidative respiration to produce ATP, whereas M1 obtain energy mainly by glycolysis and M2 by enhanced oxidative metabolism (Fig. 5) (241, 242).

Importantly, the metabolic status characterizing differentially activated $\mathrm{M} \Phi$ also affects their inflammatory state; therefore, different metabolic pathways not only are a different way to produce energy but also confer peculiar phenotypes and functions to M $\Phi$ subsets. Although a growing body of evidence emphasizes the crucial role of metabolic reprogramming in $М \Phi$ activation, the signaling allowing the metabolic switch leading to M1 or M2 activation is currently largely unknown.

\section{A. M1-polarized $M \Phi$}

1. Enhanced glycolysis and reduced oxidative phosphorylation. M1 activation is associated with an oxidative phosphorylation (OxPhos) to glycolysis switch (Fig. 5). The first evidence of a higher glucose consumption in murine МФ on pathogen stimulation was obtained in 1970 (124). This was confirmed in several manuscripts describing that, in LPS/ IFN $\gamma$-polarized $\mathrm{M} \Phi$, an enhanced glycolysis fosters in- creased glucose uptake and conversion of pyruvate to lactate, the latter found in high concentrations both intra- and extracellularly; glucose is fermented to lactate, even though oxygen is sufficient to support $\mathrm{OxPhos}(241,251)$. Vice versa, glucose metabolism remains unaltered in IL-4/IL-13stimulated $M \Phi$ and dendritic cells $(87,164,285,355)$. In line with this, although not fully elucidated, endogenous GLUT4 seems to be crucial for M1 activation $(238,251)$.

In addition, metabolomic screenings and cDNA microarray gene expression analysis have shown that M1 upregulate glycolytic genes within $24 \mathrm{~h}$ after LPS stimulation, whereas they downregulate the mitochondrial ones. This is associated with a reduction of the respiratory chain activity evaluated by oxygen consumption rate (OCR), which is a measurement of cellular oxidative metabolism. It is also associated with an increased extracellular acidification rate, which is an indication of the glycolytic rate (238); in fact, extracellular $\mathrm{H}^{+}$excretion derives both from anaerobic glycolysis-produced lactate (glucose is converted to lactate ${ }^{-}$and $\mathrm{H}^{+}$; glycolytic acidification) and from tricarboxylic acid (TCA) cycle-derived $\mathrm{CO}_{2}$ (exported $\mathrm{CO}_{2}$ is hydrated to $\mathrm{H}_{2} \mathrm{CO}_{3}$, which then dissociates to $\mathrm{HCO}_{3}{ }^{-}$and $\mathrm{H}^{+}$; respiratory acidification).

Although the contribution of $\mathrm{CO}_{2}$ to extracellular acidification is often considered negligible, the proportions of glycolytic and respiratory acidification vary depending on the experimental conditions (221). To sum up, M1 polarization is characterized not only by enhanced glycolysis but also by repression of mitochondrial OxPhos $(263,264)$. M1 polarization also reduces the $\mathrm{NAD}^{+} / \mathrm{NADH}$ ratio in mice, this being in line with reduced oxidative respiration and NADH oxidation (Fig. 5) (214).
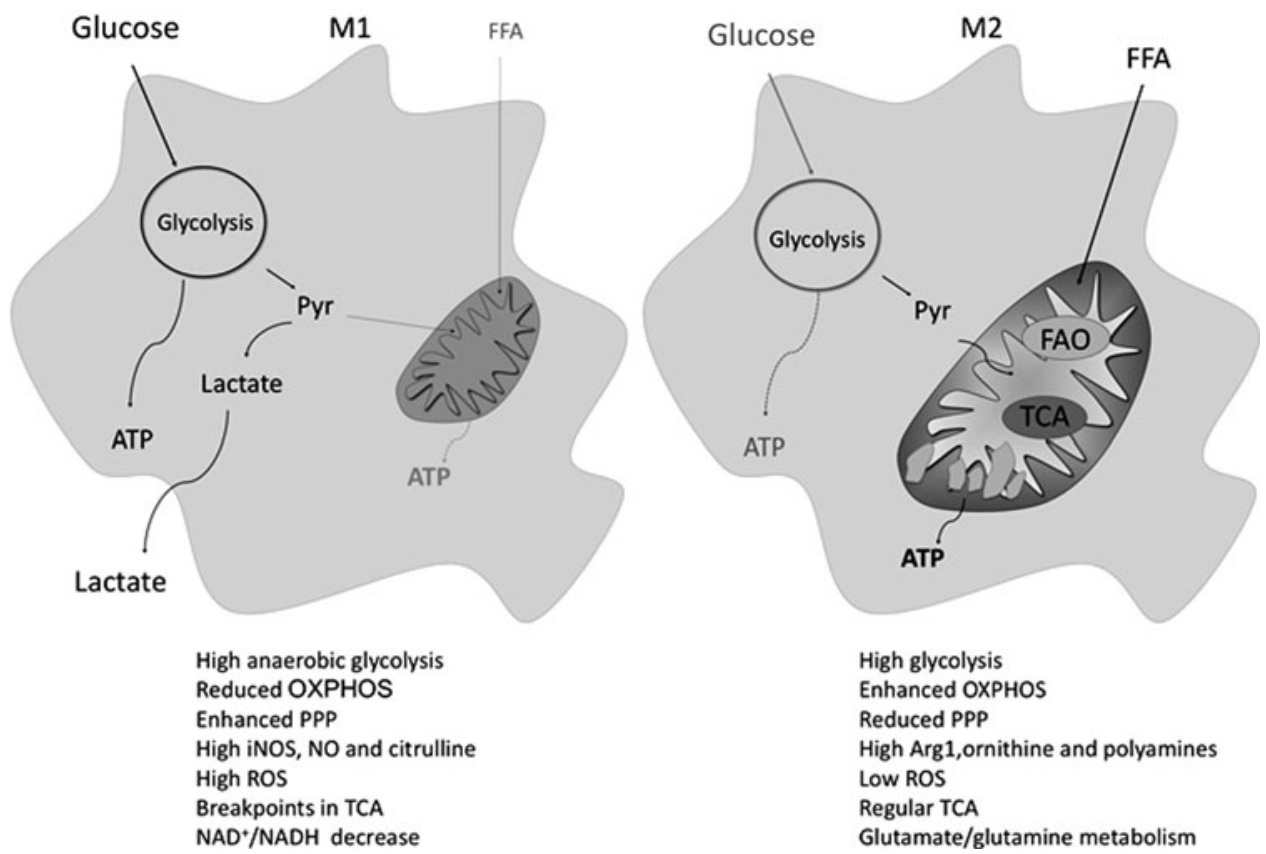

FIG. 5. Main metabolic features of M1- and M2-polarized MФ. M1 МФ obtain energy mainly by glycolysis with production and release of lactate, whereas M2 mostly rely on enhanced mitochondrial oxidative respiration based on both glucose and FFA utilization; this causes a lower NAD ${ }^{+} / \mathrm{NADH}$ ratio in $\mathrm{M} 1$. In M2, the TCA cycle is regular, whereas M1 are charcterized by breakpoints in the TCA. In M1, the PPP is more active, along with a higher production of ROS, compared with M2 subsets. Finally, M1 activate iNOS and produce high amounts of NO and citrulline, whereas M2 robustly activate Arg1 ornithine and polyamines. The glutamate/glutamine metabolism plays a crucial role in supporting M2 polarization. FFA, free fatty acid; PPP, pentose phosphate pathway; ROS, reactive oxygen species; TCA, tricarboxylic acid. 
As recently reviewed by Van den Bossche et al. (355), glycolysis is also necessary for M1 activation given that it provides signals driving this polarization route, with glycolytic enzymes being crucial in supporting pro-inflammatory function. For example, the glycolytic activator 6-phosphofructo-2-kinase/ fructose-2,6-biphosphatase 3 (PFKFB3) enhances the ability of murine $\mathrm{M} \Phi$ to remove virus-infected cells (147). Moreover, when glycolysis is limited, glyceraldeyhe phosphate dehydrogenase (GAPDH) is not fully engaged in this cycle and might inhibit $T N F \alpha$ and $I F N \gamma$ translation by binding their mRNA.

Therefore, glycolysis is a metabolically regulated signaling mechanism that is required to control cytokine production $(38,211)$. Moreover, $\alpha$-enolase is expressed on the human M1 surface where it stimulates the production of pro-inflammatory cytokines (9), and pyruvate kinase M2 (PKM2) acts directly on HIF- $1 \alpha$ and upregulates $I L-1 \beta$. Further, through the activation of the eukaryotic translation initiation factor 2 alpha kinase 2 which modulates the inflammasome-PKM2 also indirectly promotes pro-IL-1 $\beta$ activation in mice (256). Finally, it has also been suggested that in coronary artery disease patients, over-utilization of glucose drives ROS production, leading to PKM2 nuclear translocation where it phosphorylates and activates STAT3 to boost the expression of $I L-1 \beta$ and $I L-6$ (310).

Glycolysis is required to induce and sustain a proinflammatory status also in other immune cells such as dendritic ones $(86,206,263,264)$. In fact, glycolysis produces low amounts of energy compared with OxPhos (2 ATP/ glucose vs. around $30 \mathrm{ATP} /$ glucose). However, it can be quickly activated, provides rapid energy, and reduces production of intermediates. As such, it has been suggested as crucial for acute bacterial killing in highly proliferating bacterial infection $(164,332)$.

2. Pentose phosphate pathway. Classical M1 activation also enhances the pentose phosphate pathway (PPP), branch- ing from glycolysis and essential for NADPH production used to produce both ROS (by NOX) and NO (Fig. 6). Through the PPP, erythrose (precursor of amino acids) and ribose (nucleotide synthesis intermediate) are also synthetized by glucose $(236,355)$.

3. Breakpoints in the TCA cycle and TCA intermediates accumulation. Another typical consequence of the metabolic reprogramming characterizing M1 polarization is a flux discontinuity at several levels of the Krebs cycle, as demonstrated in murine M $\Phi$ (Figs. 6 and 7) (145). Such interruptions lead to the accumulation or reduction of some TCA intermediates, which influence the inflammatory response. However, the results of these studies are controversial due to the quick changes in metabolism and to the anaplerotic reactions feeding the TCA cycle. Notably, the TCA cycle fueled by both pyruvate and glutamine is globally maintained on LPS stimulation, whereas OxPhos decreases and NADH excess might possibly be converted into NADPH to support NOX activity during phagocytosis, as found in murine $\mathrm{M} \Phi$ cell lines (207).

\section{a. Citrate/isocitrate level}

(1) A robust increase of citrate occurs in M1 (Fig. 7). Citrate might translocate from the mitochondria to the cytosol by the citrate carrier (CIC; which is upregulated in M1) and be transformed into oxalacetate and acetyl coenzyme A (acetyl-CoA) by the ATP-citrate lyase (Fig. 7). Flux discontinuity at the citrate level might be responsible for the increased synthesis of acetyl-CoA, allowing higher synthesis of fatty acids (FA) and prostaglandins, characterizing the M1 subset.

FA are necessary for M1 to reorganize the plasma membrane and rapidly produce organelles that are crucial for cytokine synthesis and secretion, as

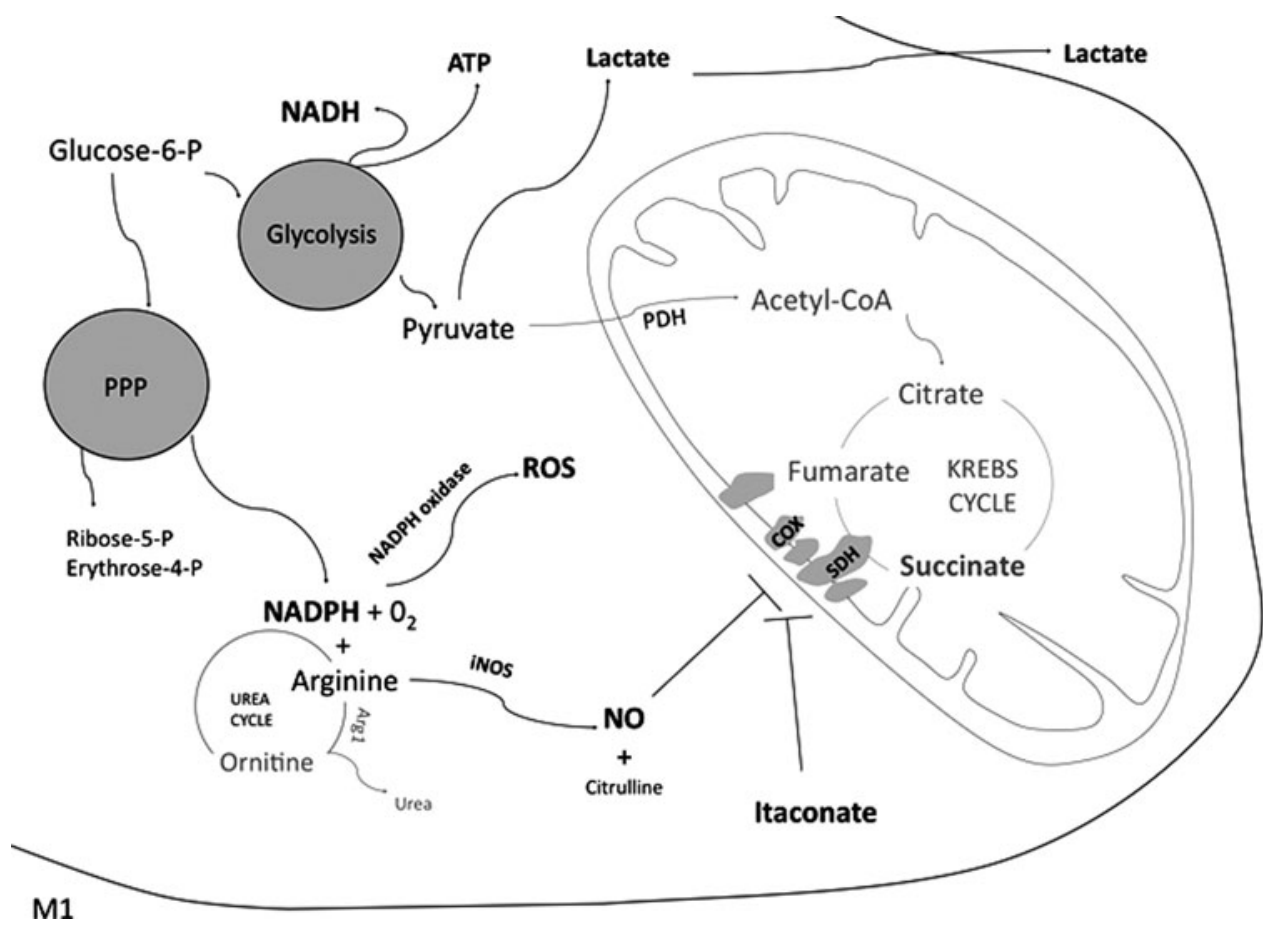

FIG. 6. Glycolysis, PPP and NO production in M1 subsets. Glycolysis in M1 is the main route of ATP and NADH production; based on this, lactate is over-produced both intra- and extracellularly. The PPP, branching from glycolysis, is also upregulated in M1. PPP is crucial for NADPH production, which is necessary for ROS production by NADPHoxidase. Moreover, NADPH is used, together with arginine and $\mathrm{O}_{2}$, to produce $\mathrm{NO}$ by iNOS activation. NO and itaconate have been suggested to reduce the oxidative respiration by inhibiting COX and SDH. Moreover, also erythrose and ribose are synthetized by glucose through the PPP. COX, cytochrome $c$ oxidase; SDH, succinate dehydrogenase. 


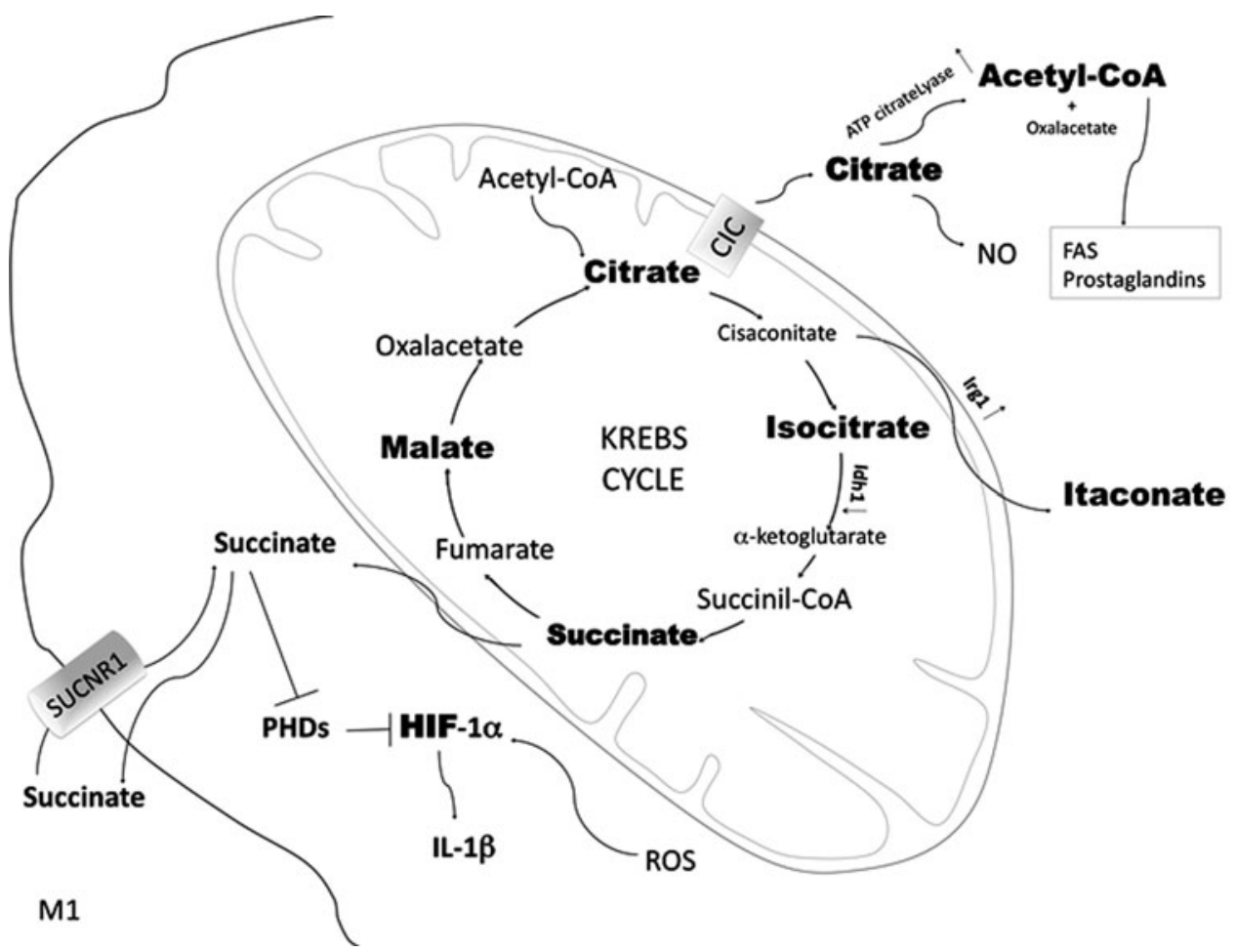

FIG. 7. Breakpoints in the TCA cycle in M1. Some breakpoints in the TCA cycle characterize the M1 subsets. Citrate levels increase in M1 MФ; citrate can translocate to the cytosol by means of the CIC and be metabolized by the ATP citrate lyase in oxalacetate and acetyl-CoA. Increased levels of acetyl-CoA might be used for FAS as well as for prostaglandin synthesis, both increasing in M1. Citrate is also used for the production of NO. The microbial agent itaconate has also been found at a high concentration in M1. Itaconate is formed by decarboxylation of cis-aconitate that is mediated by the enzyme Irg1, which is upregulated in M1. Another breakpoint within the TCA cycle flow occurs at the Idh1 level, the enzyme catalyzing the reaction from isocitrate to a-ketoglutarate. Due to Idh1 downregulation, high levels of isocitrate are found in M1. Another flux discontinuity in the TCA cycle occurs at the SDH level, leading to a high concentration of succinate, which is able to inhibit PHDs and, as a consequence, HIF- $1 \alpha$ is not degraded and drives the transcription of glycolitic genes as well as of the inflammatory cytokine IL- $1 \beta$. Therefore, succinate has been considered an inflammatory signal that might also be released and act on other cells via the G-coupled SUCNR1, thus increasing HIF- $1 \alpha$-dependent IL- $1 \beta$ expression. acetyl-CoA, acetyl coenzyme A; CIC, citrate carrier; FAS, fatty acid synthesis; HIF, hypoxia inducible factor; Idh1, isocitrate dehydrogenase 1; Irg1, immunoresponsive gene 1; PHDs, prolyl hydroxylases; SUCNR1, succinate receptor 1.

demonstrated both in vivo and in vitro $(86,87,208$, $355,378)$. FA synthesis occurring in M1 demonstrates that glycolysis is boosted and oxygen consumption is decreased yet not abrogated, as observed in vitro (214). Citrate is also critical for the production of pro-inflammatory mediators such as NO (140); if CIC or ATP-citrate lyase are inhibited, NO and ROS production by M1 is blocked (140, 141).

(2) Deriving from the TCA cycle intermediate cisaconitate (which, in turn, derives from the accumulated citrate), the itaconic acid is also highly concentrated in murine M1 (Fig. 7) (320). Pyruvate dehydrogenase $(\mathrm{PDH})$ is necessary to obtain citrate (and, in turn, itaconate) from pyruvate (355). Itaconic acid acts as a microbicidal agent disrupting the glyoxylate cycle used by some pathogens (e.g., Salmonella enterica), but not by mammalian cells (209). Itaconic acid is secreted on M1 activation. In addition, Michelucci et al. have found that silencing the immunoresponsive gene $1(\operatorname{Irg} 1)$ - the enzyme catalyzing the decarboxylation of cis-aconitate to itaconate-reduces $М \Phi$ antimicrobial activity (209,
331). Indeed, Irg1 is robustly upregulated in M1, thus linking the accumulation of citrate and succinate (Fig. 7) (61, 168).

(3) More recently, disclosure of the metabolic rewiring typical of the M1- and M2-polarized states obtained by high-throughput metabolic and transcriptional data profiling (CoMBI-T analysis), besides confirming previous findings, has highlighted that M1 polarization is characterized by TCA cycle breakpoint at the isocitrate dehydrogenase 1 (Idh1) level (Fig. 7); M1 display higher levels of isocitrate compared with M0 МФ and this was likely due to transcriptional downregulation of $I d h I$, the enzyme catalyzing the isocitrate to $\alpha$-ketoglutarate reaction.

\section{b. Succinate level}

(1) Another TCA flux discontinuity occurs at the succinate dehydrogenase (SDH) level; the succinate-tofumarate transition is impaired, and the steady-state concentration of succinate and malate increases in M1. Data suggest that malate accumulates because it de- 
rives from the aspartate-arginosuccinate cycle, an anaplerotic set of reactions connecting the urea cycle (arginine-ornithine-citruline conversion) and NO production with the Krebs cycle (Fig. 8) (145). This was confirmed by inhibition of glutamic oxaloacetic transaminase 2-mediated aspartate production, which reduced NO production and iNOS expression, in turn decreasing IL-6 and blocking M1 conversion.

Based on these data, NO seems to be largely produced through the aspartate-arginosuccinate shunt. As shown in cultured murine dendritic cells, NO contributes to suppressing mitochondrial respiration by competing with oxygen and inhibiting SDH (87). To summarize, it seems that in M1, the aspartatearginosuccinate shunt allows TCA cycle anaplerosis, which is useful in the context of the breakpoint at the SDH level. This shunt coordinates the NO cycle with TCA cycle anaplerosis (Fig. 8) (145).

(2) Further, accumulated succinate, similar to NO, inhibits PHDs (Fig. 7). Usually, in the presence of oxygen, the $\alpha$-ketoglutarate-dependent dioxygenases PHDs hydroxylate HIFs, targeting them for proteasomal degradation. At low $\mathrm{pO}_{2}$, PHDs are inhibited; $\mathrm{HIF}-1 \alpha$ is not degraded, thus stimulating glucose uptake, glycolytic genes, and $I L-1 \beta$ transcription while inhibiting pyruvate oxidation and favoring lactate reduction. PHDs inhibition by succinate stabilizes HIF$1 \alpha$ also in the presence of oxygen (334); succinate is, therefore, considered an inflammatory signal since HIF- $1 \alpha$ plays a crucial role in orchestrating part of the M1 polarization by promoting glycolysis, GLUT4 and $I L-1 \beta$ expression, and $\mathrm{M} \Phi$ migration.

Glycolysis is crucial for MФ migration, whose inhibition suppresses systemic inflammation in vivo (308). Notably, in LPS-treated MФ, inhibiting gly- colysis by 2 -deoxy-D-glucose (2DG) reduces $I L-1 \beta$ (most likely due to succinate accumulation) but not $T N F \alpha$ expression, thereby demonstrating the specificity of the effect of succinate on IL-1 $\beta(214,238,334)$.

(3) Succinate might also be released by M1, acting extracellularly via the succinate receptor $1 / \mathrm{G}$-protein coupled receptor-91 (SUCNR1/GPR91) expressed in many tissues. Recycling succinate induces a feedforward loop of pro-inflammatory $M \Phi$ activation, which increases HIF- $1 \alpha$-dependent $I L-1 \beta$ expression. In fact, SUCNR1 synergizes with TLR on both human and murine dendritic cells to enhance the functions associated with antigen presentation (Fig. 7) (185, 290). Moreover, an LPS-dependent succinylation of numerous proteins-whose consequences are unknown-has been reported (335).

(4) An increased succinate oxidation via SDH on M1 polarization, as observed in murine BMDM, has also been proposed (214). In addition to HIF- $1 \alpha$ stabilization, succinate might have another inflammatory critical role; M1 polarization seems to drive mitochondrial membrane hyperpolarization (glycolysis supporting ATP generation), which, paired with SDH-mediated succinate oxidation to fumarate, leads to ROS generation (and, in turn, to IL-1 $\beta$ expression) via reverse electron transport through complex I rather than activating the conventional electron transport $(214,251,317)$.

This hypothesis suggests a repurposing of mitochondria from ATP synthesis to ROS production, which promotes a pro-inflammatory state (214). This is accompanied by mitochondrial supercomplex destabilization; complexes I, II, and IV can accumulate as supercomplexes, improving coupling and reducing ROS formation. However, complex I, and, as a consequence, the whole supercomplex, is destabilized when $M \Phi$ are

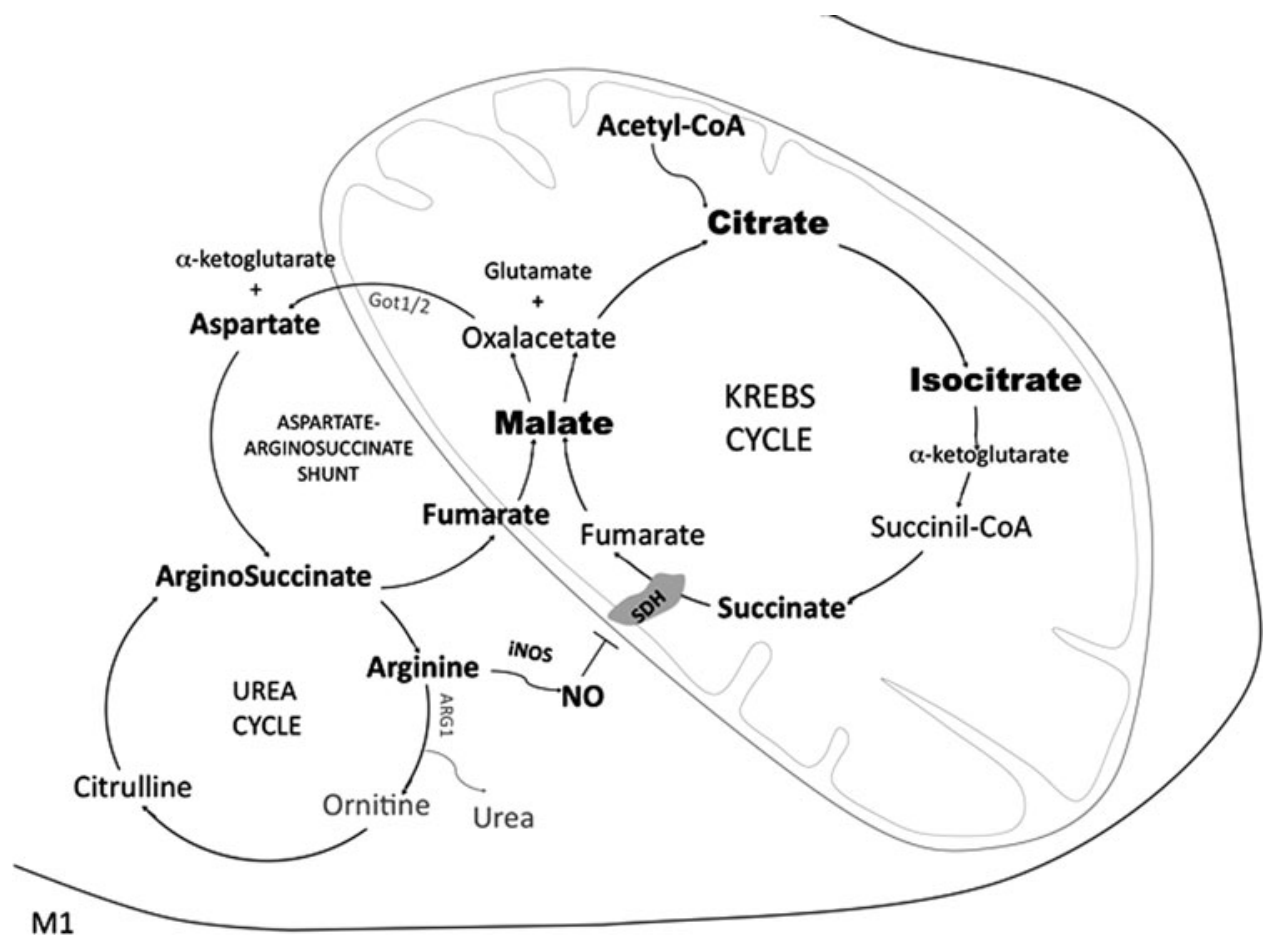

FIG. 8. The aspartatearginosuccinate shunt allows anaplerosis of the TCA cycle in M1. Considering that the succinate-to-fumarate transition is impaired, the concentration of succinate and malate increases in M1. It has been suggested that malate accumulates because it derives from the aspartatearginosuccinate cycle that connects the urea cycle and iNOS-dependent NO production with the TCA cycle. NO is largely produced through the aspartate-arginosuccinate shunt. NO inhibits the SDH and reduces mitochondrial respiration. 
activated by bacteria; this activates $\mathrm{SDH}$, which seems necessary for the control of bacteria (214), although the role of SDH and its link with IL- $1 \beta$ production is far from being clear. Succinate oxidation also leads to decreased anti-inflammatory gene expression; in line with this, inhibiting succinate oxidation by dimethyl malonate promotes an anti-inflammatory outcome.

To summarize, M1 polarization is characterized by enhanced glycolysis and PPP, whereas OxPhos decreases. Glycolysis is additionally necessary for M1 activation by providing signaling mediators driving it. Flux discontinuities on several levels of the Krebs cycle are also features of M1 polarization and lead to a robust increase of proinflammatory succinate and citrate, Irg1, isocitrate, and microbicidal itaconic acid and to the downregulation of Idh1. The variation of Irg1 and itaconate concentration represents a strong marker of M1 polarization similar to iNOS activation and NO over-production (see section IV.C). Specifically, PFKFB3, PMK2, $\alpha$-enolase, citrate, succinate, and itaconate are not only consequences but also causal of M1 polarization.

\section{B. M2-polarized $M \Phi$}

1. Enhanced OxPhos. Differently from M1, alternatively activated murine M2 have an intact TCA cycle and an efficient OxPhos supporting their energy demands and phenotype (Figs. 5 and 9) (357). Based on some authors' findings, glucose uptake also increases in M2 compared with untreated $\mathrm{M} \Phi$ but is lower than in M1, and it is mainly oxidized by mitochondria; OxPhos accounts for higher but slower ATP generation, which can be sustained for a longer period compared with glycolysis (355). This is useful for the resolution of inflammation and also against prolonged parasite infections. OxPhos requirement for M2 polarization has been revealed by oligomycin-mediated inhibition of ATPsynthase and OCR, blocking IL-4-mediated M2 polarization. Also, OxPhos inhibition by rotenone impairs M2 polarization in murine BMDM (126).

2. Reduced PPP. The carbohydrate kinase-like (CARKL) protein is a repressor of M1 activation both in human and in murine MФ. In fact, CARKL phosphorylates the PPP intermediate sedoheptulose in sedoheptulose-7-P, thus stimulating the nonoxidative phase of this cycle; this reduces NADPH production and counteracts ROS production typical of M1. CARKL is critical for PPP regulation; its expression increases in M2 whereas it decreases in M1 (Fig. 9) (126).

3. Free fatty acid $\beta$-oxidation and glycolysis requirement in differentially polarized $M \Phi$ is controversial. The strict requirement of free fatty acid (FFA) $\beta$-oxidation (FAO) for M2 polarization has recently become a matter of debate (134, 237, 332, 355, 357). By using BMDM, Vats et al. suggested that FAO is crucial for M2 polarization; based on their data, FFA uptake, and FAO increase in M2 compared with untreated MФ and M1; whereas IL-4-stimulated MФ upregulate

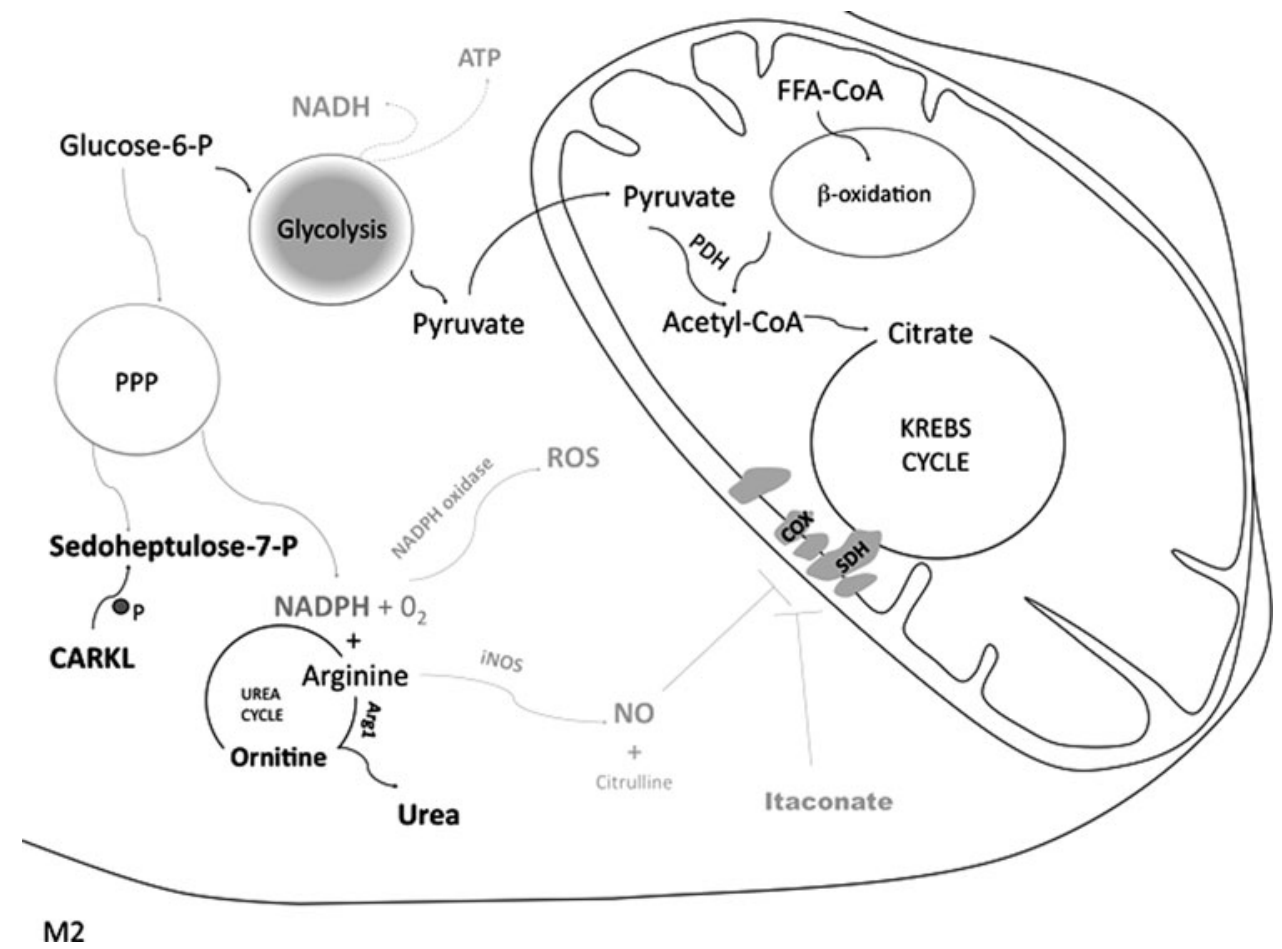

FIG. 9. Enhanced OxPhos, upregulated Arg1, and reduced PPP in M2 polarization. In M2, the glycolysis is still greatly used by the cell, but pyruvate is mainly metabolized in acetyl-CoA, which is also obtained from FAO and feeds the TCA (Krebs) cycle and, in turn, the respiratory chain. Another feature of anti-inflammatory MPs is the upregulation of the enzyme Arg1, which is inducible and competes with iNOS for their common substrate L-arginine to produce ornithine and urea, thus reducing the production of NO. The CARKL phosphorylates the PPP intermediate sedoheptulose in sedoheptulose-7-P, stimulating the nonoxidative phase of this cycle and reducing NADPH, ROS, and NO production typical of M1 activation. CARKL, carbohydrate kinase-like; FAO, FFA $\beta$ oxidation; OxPhos, oxidative phosphorylation. 
acyl-CoA dehydrogenases and enoyl-CoA hydratases involved in FAO (357). mRNAs of PPAR $\gamma$-coactivator- $1 \beta$ $(P G C-1 \beta)$ and of genes involved in FFA uptake, transport, and oxidation-for example, lipoprotein lipase, fatty acid transporter $(C D 36 / F A T)$, carnitine palmitoyltransferase 1 (CPT1), medium chain acyl-CoA dehydrogenase, long chain acylCoA dehydrogenase, and PPAR - are also upregulated in M2 and downregulated in LPS/IFN $\gamma$-polarized M1 (357).

Triglycerides lysosomal lipolysis, after CD36-mediated lipoprotein uptake, fuels an intense OxPhos; lipolysis was found to be essential to allow oxidative metabolism and M2 polarization, both in humans and in mice (134). In line with this, and further corroborating the hypothesis of the key role of FAO in M2 polarization, PPAR $\gamma$, necessary for mitochondrial function and FAO, enhances and is crucial for M2 activation and consequent reduction of inflammation; in fact, its inhibition impairs alternative polarization $(163,249)$.

Consistently, M2 activation requires PGC- $1 \beta$ and the induction of the transcription factor STAT6, enhancing mitochondrial respiration through the upregulation of $P P A R \gamma$ but also $P G C-1 \alpha$ and $P G C-1 \beta$, the master regulators of mitochondrial biogenesis (237). Accordingly, some authors have found that oxidative metabolism increases on M2 polarization and etomoxir-mediated FAO inhibition, similar to the inhibition of OxPhos by oligomycin or by the mitochondrial uncoupler, carbonyl-cyanide-4-trifluoromethoxy-phenylhydrazone, which completely abolishes IL-4-induced polarization as highlighted by the downregulation of the M2 polarization markers Arg1, Dectin-1, CD301, CD206, and RELM $\alpha(134,238)$.

More recently, however, the strict FAO requirement for M2 polarization has become controversial (208); even though FAO and oxygen consumption increase in human M2, the global oxidative metabolism - not specifically FAO-might be crucial for M2 polarization, since glucose can be used to fuel OxPhos. In fact, although FAO decreases during M1 polarization, it has been demonstrated, in both murine and human $\mathrm{M} \Phi$, that etomoxir-mediated FAO inhibition does not block mitochondrial respiration and does not inhibit M2 polarization $(237,332,355)$. Notably, suppression of glucose oxidation (not FAO suppression) would inhibit M2 polarization (332); this hypothesis is corroborated by the observation that $2 \mathrm{DG}$-dependent glycolysis-inhibition-similar to mitochondrial ATPsynthase inhibition by oligomycinblocks respiration and $\mathrm{M} 2$ polarization, as observed both in human and in murine M $\Phi$ both in vivo and in vitro $(63,246$, $353,397)$.

Glucose might fuel the TCA cycle for mitochondrial respiration in M2 and, as a consequence, glycolysis might be crucial not only for M1 but also for M2 polarization. In humans, FAO seems to be dispensable for M2 polarization; indeed, IL-4 leads to an unchanged expression of PGC- $1 \alpha / \beta$ and to moderate changes in mitochondrial oxidative metabolism and FAO rate, thus highlighting a possible key difference between mice and humans $(97,332)$. Moreover, CPT2 knockdown and FAO disruption allows M2 activation, again demonstrating that FAO is dispensable for $\mathrm{M} 2$ activation (244); accordingly, some manuscripts report that FAO inhibition does not influence STAT6 phosphorylation and PGC-1 $\beta$ expression that are necessary for M2 polarization $(238,357)$.

Notably, the effect of etomoxir-mediated FAO inhibition is highly controversial not only because of differences among mice and humans but also because, as discussed in Namgaladze and Brüne (238), different concentrations of etomoxir trigger different effects. Low concentrations of etomoxir inhibit $90 \%$ of FAO whereas respiration is only slightly affected; this means that cells shift to another metabolism to fuel OxPhos. High etomoxir concentrations block FAO and also decrease respiration by $50 \%$; they further reduce the expression of IL-4-target genes also in $C P T 2^{-/} \mathrm{M} \Phi$.

Moreover, genetic ablation of the FA transport protein 1 (FATP1) has been used to get insights into the role of FAO, without clarifying the issue. In fact, FATP1 deletion in murine MФ triggers an FAO-to-glycolysis switch with iNOS upregulation and Argl downregulation, without altering the expression of M1 surface markers, including CD80, CD86, and major histocompatibility complex II, MHC-II (151). Also, the role of the adipocyte triglyceride lipase fueling FAO in MФ is controversial (238).

Interestingly, it has also been proposed that IL-4 acts by activating AKT and, in turn, mammalian target of rapamycin (mTOR) complex-1 (TORC1), which stimulates glucose metabolism (63). Other authors reported that IL-4, in association with M-CSF, also acts through mTORC2 and IRF4 to increase glucose metabolism in murine $\mathrm{M} \Phi$, both in vitro and in vivo (135). This pathway resulted critical for alternative MФ activation; in fact, deletion of Rictor (a component of mTORC2) reduces glycolysis and M2 activation. In conclusion, the necessity for glycolysis might not be typical of M1, but, vice versa, might be crucial for both inflammatory and anti-inflammatory responses. mTOR signaling regulation of MФ polarization suggests that prolonged starvation might lead to an interesting interplay between mTOR signaling, metabolism, and MФ polarization, which needs to be explored (30).

4. Glutamine-related metabolism. Recently, the glutamine/ glutamate-related metabolism and the UDP- $N$-acetylglucosamine (UDP-GlcNAc) biosynthesis, through the hexosamine biosynthetic route, have been found to be enhanced and critical for M2 polarization in murine BMDM in vitro (145). Also, high levels of UDP-glucose and UDP-glucoronate characterize this polarization status (Fig. 10). Several studies confirm the crucial role of glutamine in the TCA cycle for M2 activation (124, 242, 285). The importance of glutamine-dependent pathways might also be associated with the requirement of UDP-GlcNAc as a sugar donor for $N$-glycosylation, possibly to properly fold and export cell surface or secretion proteins (145). $N$-glycosylation is crucial for M2 activation, since highly glycosylated lectin/ mannose receptors are the most typical markers for M2 polarization; the $\mathrm{N}$-glycosylation inhibitor tunicamycin inhibits the expression of the M2 markers RELM $\alpha$, CD206, and CD301.

Moreover, UDP-GlcNAc might be used as a sugar donor for $O$-glycosylation, another pathway connecting cellular metabolism with signaling $(145,379)$. Finally, glutamate/ glutamine support an active TCA (Fig. 10). In general, the availability of amino acids and nutrients in the microenvironment has a profound impact on metabolism and, by extension, on function. Metabolic intermediates are not just a source of energy, but they can also be directly implicated in the definition of a particular M $\Phi$ phenotype (117). For example, in lymphocytes, the inability to transport some amino 


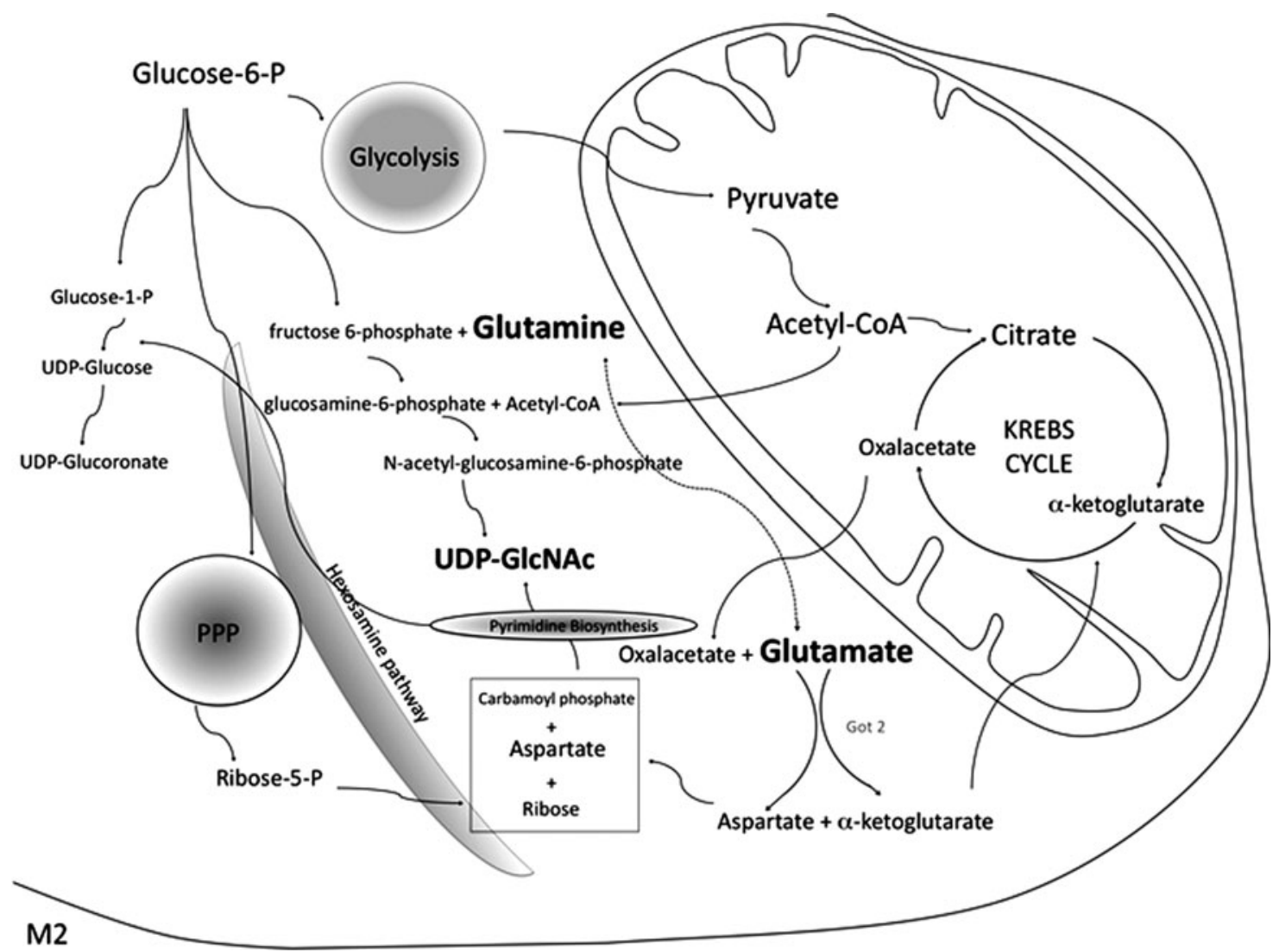

FIG. 10. The glutamine/glutamate-related metabolism and the UDP-GlcNAc biosynthesis are critical for M2 polarization. Glutamine plays a key role in the activation of the M2-polarization program. UDP-GlcNAc is necessary as a sugar donor for $\mathrm{N}$-glycosylation and $O$-glycosylation. UDP-GlcNAc originates through the contribution of glutamine and glutamate through the hexosamin pathway; from glucose-6-P, fructose-6-P is formed and by addition of glutamine, is transformed into glucosamine-6-phosphate, undergoing an addition of acetyl-CoA to produce $N$-acetyl-glucosamine-6phosphate. With the contribution of ribose (obtained by the PPP), aspartate (obtained by a reaction involving glutamate), carbamoyl phosphate, and through the route of the pyrimidine biosynthesis, UDP-GlcNAc is formed. High levels of UDPglucose and UDP-glucoronate characterize the M2 polarization status as well. Glutamate/glutamine support an active TCA, by, for example, producing $\alpha$-ketoglutarate. UDP-GlcNAc, UDP- $N$-acetylglucosamine.

acids (including glutamine) leads to inflammation (251). An extensive literature on the impact of amino acid deficiency on immune cells has been recently reviewed $(192,233)$.

In synthesis, M2 are characterized by an intact TCA cycle and a high and efficient OxPhos, which is required for M2 polarization; whereas PPP decreases and CARKL, a repressor of M1 activation, is upregulated. Glutamine is also crucial for M2 activation; moreover, PPAR $\gamma$ fosters M2 polarization, and GAPDH inhibits inflammation. Notably, the key role of FAO in M2 activation has recently been questioned, since it has been proposed that the global oxidative metabolism, also fueled by glycolysis, but not specifically FAO, is crucial for M2 polarization.

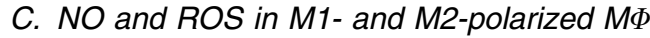

1. NO/iNOS and ROS in M1. Oxidative stress is associated to inflammation; classically activated M1 recognize invading microbes or cancer cells, engulf them into phagosomes, and destroy them on phagosomes-lysosomes fusion. M1 bactericidal action mainly relies on ROS production occurring in phagolysosomes, and on the production of the cytosolic diffusible NO, which reacts with phagolysosomal ROS to produce highly toxic species. NO and ROS account not only for M1 toxic, antimicrobial, and antitumor effects but also for the redox signaling that modulates many transcriptional events; this mainly occurs at low intracellular free radical levels, when changes elicited by ROS and NO are subtle and reversible.

Vice versa, high NO and ROS levels induce oxidative stress, which is a disturbance of the pro-oxidant/antioxidant balance occurring when the redox state redox systems shifts to the oxidized state. If prolonged, this condition is toxic and bactericidal and leads to protein, DNA, and lipid oxidation and damage. NO and ROS toxicity depends not only on their concentration but also on the type of oxidative species produced (see the subsequent paragraphs within this section).

A key effector molecule preferentially expressed in inflammatory M1 is the cytokine-inducible iNOS (NOS2); it produces NO from L-arginine, thus accounting for a prolonged period of NO production, which, therefore, reaches a high concentration and elicits bactericidal and tumoricidal effects (Fig. 6) (130, 231, 234). iNOS requires L-arginine, $\mathrm{NADPH}$, and molecular oxygen to generate $\mathrm{NO}$ and 
citrulline. NO might also be produced in the absence of oxygen (likely in mitochondria) from nitrite reduction caused by some heme proteins $(28,106,131)$. Two other NOS isoforms exist: endothelial NO synthase (eNOS/NOS3) and neuronal NO synthase (nNOS/NOS1), constitutively expressed and releasing continuous but low amounts of NO.

Moreover, a mitochondrial NO synthase (mt-NOS) has been hypothesized; although it awaits characterization, it has been proposed that mt-NOS might directly interact with COX to block its activity in hypoxic conditions $(104,271)$. Notably, although production of NO by NOS is considered a coupled reaction, NOS might also produce ROS by uncoupled reactions, although their exact stoichiometry awaits further clarification $(98,302)$.

The NO moiety is added to thiols, secondary amines, or hydroxyl groups of cellular macromolecules by nitrosative reactions. In particular, $S$-nitrosylation mostly occurs at low $\mathrm{NO}$ concentrations and is the reversible addition of an NO moiety to sulfhydryl residues, generating $S$-nitrosothiol derivatives characterized mostly by regulative roles (220). NO binds heme and nonheme iron targets (Fe-nitrosylation) and inhibits the electron transport chain (ETC) both reversibly, by competing with oxygen for the COX heme, and irreversibly, by reacting (mostly as peroxynitrite) with iron-sulfur clusters in complexes I and II (229). In highly inflammatory conditions, NO might also impair the ETC of the cell in which it is produced, this being typical of M1.

Not only COX, SDH, and, as a consequence, the whole oxidative metabolism are directly inhibited by NO (possibly in concert with itaconate; see section IV.A.3.a), but also PHDs are inhibited by NO, thus leading to HIF- $1 \alpha$ stabilization $(87,164,321,353)$ (see sections III and IV.A.3.a). iNOS may also generate $N$-hydroxyarginine (an inhibitor of Arg1) and the superoxide anion $\mathrm{O}_{2} \bullet^{-}$. In addition, oxidation and reduction of $\mathrm{NO}$, as well as its reaction with oxygen or $\mathrm{O}_{2} \bullet^{-}$, produces several nitrogen species (among which are nitroxyl, nitrite, nitrate, nitrogen dioxide, peroxynitrite, and nitrosoperoxocarbonate), some of which are highly reactive (28). In particular, peroxynitrite and nitrogen dioxide, deriving from the reaction of $\mathrm{NO}$ with oxygen, are strong oxidants leading to the oxidation and nitration of proteins, lipids, and DNA; nitrotyrosines are a major marker of nitroxidative stress. iNOS levels and NO concentration have been suggested to be lower in humans compared with rodents, and the mix of TNF $\alpha$ and IFN $\gamma$ used for rodents might not be sufficient to induce NO production in human MФ in vitro (28).

Besides NO, M1 generate ROS. ROS production is associated with high $\mathrm{M} \Phi$ phagocytic activity and inflammatory response, also leading to tissue damage along with cytokines and chemokines. NO and ROS combine to produce highly reactive species, thus increasing nitroxidative stress. Blocking ROS production inhibits the M1 inflammatory phenotype $(288,331)$. Although the mechanism by which $M \Phi$ generate ROS needs to be fully elucidated, ROS formation mainly derives from NOX, mitochondria, and also from NOS; indeed, as stated earlier, NOS participates in the elimination of pathogens also through direct generation of ROS (28). NOX1 to NOX5 and dual oxidases-1/2 (DUOX1 and DUOX2) are the primary sources of ROS.

NOX are transmembrane complexes that are able to transport electrons across membranes, thus leading to the reduction of oxygen into superoxide $\mathrm{O}_{2} \bullet^{-}$. When this occurs across the phagocytic membrane, $\mathrm{O}_{2} \bullet^{-}$is released into the vesicles. ROS production in $\mathrm{M} \Phi$ on pathogen recognition is predominantly attributable to NOX2, whose subunits are assembled into phagolysosome membranes on stimuli such as IFN $\gamma$ and TLR activation $(31,331)$. Interestingly, a direct interaction between TLR4 and NOX4 has been reported (331). NOX2 directly produces $\mathrm{O}_{2} \bullet^{-}$, which is converted to other ROS, for example, its dismutated product hydrogen peroxide $\left(\mathrm{H}_{2} \mathrm{O}_{2}\right)$, the highly reactive hydroxyl radical $\left(\mathrm{OH}^{\bullet}\right)$ produced by the Fenton chemistry, and associated species.

As a consequence, the targets engulfed by $М \Phi$ become submerged into a mixture of toxic oxidants. NOX also contribute to lowering phagolysosome $\mathrm{pH}$, thus favoring the activity of lysosomal proteolytic enzymes contributing to M1 antimicrobial action. TLR4 stimulation also leads to mitochondrial ROS generation from complex I through an unknown mechanism. Moreover, in M1, another mechanism dependent on succinate oxidation by SDH has been proposed for ROS production (see sections III and IV.A.3.b) (214); even the enzyme Irg1 and citrate induce ROS production (see sections III and IV.A.3.a) (118, 140, 141).

2. NO/Arg1 and ROS in M2. After the inflammatory phase, M $\Phi$ switch to the M2 phenotype and release IL-10 and TGF- $\beta$ reducing inflammation; it also reduces ROS and NO production by upregulating, among other mechanisms, Arg1. Arg1 is inducible and competes with iNOS for their common substrate L-arginine to produce ornithine, polyamines, and urea (Fig. 9) (35, 142). By limiting L-arginine availability, cytosolic Arg1 decreases the production of $\mathrm{NO}(27,62,83$, 217). Ornithine is the starting point of polyamine synthesis (putrescine, spermidine, and spermine), contributing to the stabilization of DNA and promoting collagen synthesis and fibrosis, thus enhancing tissue repair.

Depending on the content of GSH, M1 and M2 have been defined as oxidative and reductive $\mathrm{M} \Phi$, respectively, confirming crucial and specific redox mechanisms in $М \Phi$ (331). Decreased ROS production, for example, by NOX2 inhibition, is necessary for M2 polarization. As stated earlier, NADPH and ROS production is reduced by CARKL, which contributes to M2 polarization. The interaction between MФ and apoptotic bodies triggers the binding of the protein SYNCRIP to the NOX2 mRNA, thereby leading to its instability and favoring the M2 phenotype (166); this effect is also achieved by apocynin, an NOX inhibitor, and by mutation of the NOX subunit $p 47^{\text {phox }}$ (331). Moreover, differently from M1, extracellular ATP seems to block IL- $1 \beta$ in M2 by inhibiting inflammasome and ROS production (331).

3. Redox signaling in M1 and M2. Redox signaling in classical versus alternative $\mathrm{M} \Phi$ polarization is far for being straightforward. Through transcription factor $S$-nitrosylation, NO regulates gene expression of not only pro-inflammatory but also respiratory chain and cell cycle genes (20). Although it is awaiting further clarification, along with NF- $\kappa \mathrm{B}$-induced iNOS expression there is also evidence of ROS and reactive nitrogen species (RNS)-mediated NF- $\kappa \mathrm{B}$ activation and M1 polarization (28). NO activates NF- $\kappa$ B likely by $S$ nitrosylation; however, long-lasting $\mathrm{NO}$ exposure reduces $\mathrm{NF}-\kappa \mathrm{B}$ activity, contributing to $\mathrm{M} 2$ polarization $(28,262)$. $\mathrm{NF}-\kappa \mathrm{B}$ is also modulated by ROS, as well as by AP1 and p38 MAPK $(262,277,331)$; also in this case, low levels of $\mathrm{H}_{2} \mathrm{O}_{2}$ 
enhance NF- $\kappa$ B activation, whereas high $\mathrm{H}_{2} \mathrm{O}_{2}$ levels inhibit it (28). Moreover, IkB oxidation reduces its degradation and, consequently, NF- $\kappa \mathrm{B}$ activation, thus contributing to M2 polarization (262).

On the other hand, ROS also activate the inflammasome, and many redox-sensitive proteins are crucial in the signaling triggered by inflammatory mediators $(14,65,289,331)$; indeed, NOX inhibition favors M2 polarization (51). Although controversial [see below Lo Sasso et al. (188)], the NADdependent HDAC silent information regulator 2 (SIRT2) seems to be involved in LPS-induced ROS generation and $\mathrm{NF}-\kappa \mathrm{B}$-dependent M1 gene expression, and $\mathrm{H}_{2} \mathrm{O}_{2}$ enhances M1 polarization by reducing TNFR1 shedding, which would reduce the inflammatory response [see references in Tan et al. (331)].

ROS and RNS are, therefore, crucial both for their cytotoxic effects and for signal transduction.

Notably, once generated in M1, they activate inflammatory genes but at the same time, also trigger protective mechanisms that might be necessary to allow the switch to the antiinflammatory M2 phenotype; accordingly, a genetic defect in ROS production induces a hyper-inflammatory response (331). The potential role of ROS in M2 polarization is also suggested by the inhibition of monocyte-to-M2 differentiation caused by the antioxidant butylated hydroxyanisole (396); similarly, MCP1-induced protein-mediated ROS stimulation might be necessary for M2 polarization (155).

Moreover, the strong interaction of NO with the soluble guanylate cyclase heme is an Fe-nitrosylation, which produces cGMP, found to be protective and anti-inflammatory. NO also induces PPAR $\gamma$, which antagonizes NOX2 assembly and attenuates NF- $\kappa \mathrm{B}$ formation. This might be part of the protective mechanisms readily activated by $М \Phi$ against excessive inflammation and tissue damage. In this context, $\mathrm{H}_{2} \mathrm{O}_{2}$ produced by SOD1 has been found to promote M2 polarization by activating STAT6 and reducing $T N F \alpha$ and iNOS expression; $\mathrm{H}_{2} \mathrm{O}_{2}$ acts on a critical STAT6 cysteine leading to STAT6 nuclear translocation $(128,331)$. In addition, previous reports have shown with controversial results that SOD1 modulates pro-inflammatory genes such as $T N F \alpha$, $I L-1 \beta$, and $i N O S$ and that, in other cell types, $\mathrm{H}_{2} \mathrm{O}_{2}$ might increase STAT6 phosphorylation via oxidative inactivation of the protein tyrosine phosphatase 1B (331).

Notably, free radicals produced by M1 MФ might also be toxic for M1 themselves, which are, indeed, equipped to survive the bactericidal oxidative stress occurring during classical activation. A crucial role is played by the transcription factor Nrf2 activating antioxidant genes; M1 might, therefore, survive and persist at the sites of infection and, in principle, switch to an M2 phenotype to allow tissue remodeling. M $\Phi$ protect themselves from radical toxicity also by increasing the expression of DNA repair proteins and free radical scavengers, whereas the chromatin remodeling necessary to induce MФ LPS tolerance is a redox-sensitive process $(13,31,162,279,337)$.

Further, М $\Phi$ protect themselves by generating many toxic species into micro-compartments; indeed, $\mathrm{O}_{2} \bullet-$ formation mostly occurs into phagolysosomes where $\mathrm{O}_{2} \bullet$ acts against pathogens but is separated from the rest of the cell, which is, therefore, protected. In phagolysosomes, ROS are also separated from cytosolic NO, thus preventing the production of highly reactive species deriving from the reaction of ROS with NO.

Moreover, oxidation and reduction of NO convert it into several nitrogen species whose ratio constantly changes during M1 activation and that might represent another form of protection from the high NO toxicity deriving by its reaction with ROS. Protection from NO is also achieved by $S$ nitrosylation of caspases, reducing the responsiveness to apoptotic signals. Over-production of ROS in M $\Phi$ during apoptotic cell phagocytosis is followed by attenuation of the oxidative burst by PPAR $\gamma$ activation as well as by resolvin D1 (derived from docosahexaenoic acid), which prevents M $\Phi$ death and ROS production by inactivating NOX2 $(150,174)$.

Although controversial, NO seems to accelerate the process of phagocytosis $(304,350)$. In particular, there is evidence that on $\mathrm{Fc} \gamma \mathrm{R}$ stimulation of unprimed MФ, nNOS and, to a lesser extent, eNOS produce low levels of NO that promotes phagocytosis by surrounding $\mathrm{M} \Phi$ in a paracrine manner (137). NO produced by MФ is necessary for PS externalization in dying cells through $S$-nitrosylation and inhibition of the aminophospholipid translocase $(331,351)$. Conversely, in nonactivated MФ, NO stimulates NOX-dependent ROS generation by increasing mitochondrial ROS, but not phagocytosis; NO enhances mitochondrial ROS formation by inhibiting the ETC (see section IV.C.1) (229). Intracellular ROS are also able to increase MФ phagocytic activity, and NOX2-deriving ROS seem to be necessary for apoptotic cell engulfment (but not for bacteria engulfment) $(187,229,301,331)$.

Extracellular ROS (mostly generated in the plasma membrane even by dying cells) have autocrine and paracrine signaling roles and are necessary for oxidative modification of dying cell surface molecules (e.g., oxidation of membrane proteins and lipids, such as phosphatidylserines), which are eat-me signals for MФ (331). Phagocytosis and ROS production are closely linked by a common signaling pathway; in fact, phosphatidylinositol 3,4,5-trisphosphate is necessary not only for cytoskeleton reorganization and phagocytosis but also for NOX activation and ROS production (123). ROS and NO are also associated to high $\mathrm{M} \Phi$ migration ability and to the consequent monocyte/MФ recruitment $(28,331)$.

Mitochondrial function is linked to their morphology, which depends on mitochondrial membrane fusion and fission. For this reason, their shape is associated with metabolic homeostasis and changes rapidly in response to metabolic cues (370). Mitochondrial dynamics in $М \Phi$ polarization is far from being clear; in fact, it has been shown that mitochondrial fission promotes pro-inflammatory TLR-induced $I L-12$ expression in M $\Phi$ and inhibits $I L-10$ expression through IRF1 stabilization (99) and that, controversially, defective mitochondrial fission augments inflammasome activation (260).

Although most of the metabolic properties of polarized M $\Phi$ have been found to be a consequence of polarization, it is conceivable, although not always proven, that these metabolic features, if triggered in $M \Phi$, would also be able to determine the direction of polarization. This is an important issue to be unraveled for future applications of immunometabolic therapies.

To sum it up, high ROS and NO production due to iNOS upregulation and Arg-1 upregulation-dependent ornithine and polyamine production are considered molecular signatures of M1- and M2 polarization, respectively. Decreased ROS production is also necessary for M2 polarization. 
Although a dualistic M $\Phi$ classification in M1 and M2 subtypes with peculiar metabolic patterns is schematically useful, the link between $\mathrm{M} \Phi$ polarization and metabolism, in particular the role of FAO, the differences between species and between in vitro and in vivo observations, awaits further elucidation. Indeed, as suggested earlier, polarized human M $\Phi$ might have, for some aspects, different metabolic features compared with murine $\mathrm{M} \Phi$, as observed by large-scale transcriptomic and proteomic analyses (200), raising the possibility of diverse immunometabolic therapeutical approaches between species. Moreover, the available information is mainly regarding the bipolar phenotypes deriving from in vitro LPS/IFN $\gamma$ - and IL-4-induced M $\Phi$, whereas the metabolic characterization of intermediate states is only at the beginning stage.

$\mathrm{M} \Phi$ polarization requires a dramatic genetic and metabolic re-organization, going through several steps among the proinflammatory and the anti-inflammatory extremes. $\mathrm{M} \Phi$ are extremely heterogeneous, and M1 and M2 categories are an over-simplification; in fact, M1 and M2 markers might also be expressed at the same time. Similar to other M1 and M2 features, even their glycolytic and oxidative metabolic features represent the extremes of a spectrum of several intermediate phenotypes (359).

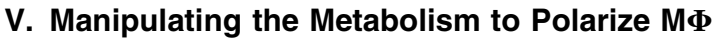

Resident $М \Phi$ show a high plasticity and adaptation to their microenvironment; $\mathrm{M} \Phi$ polarization has been suggested to be reversible, so that differentiated $\mathrm{M} \Phi$ might be reprogrammed to switch from one profile to another if transferred to a different environment $(86,169,170,335)$. However, evidence of in vivo M1-to-M2 repolarization is lacking; a recent in vivo study, performed with both murine and human $\mathrm{M} \Phi$, reported that M1 are unable to reconvert to M2, whereas M2 might be repolarized into M1 (353). The authors found that the inability to reconvert into an anti-inflammatory M2 phenotype is caused by an OxPhos inhibition occurring in inflammatory M1. More specifically, M1-produced NO impairs mitochondrial function; inhibiting iNOS during LPS/IFN $\gamma$ induced polarization recovers mitochondrial respiration and allows M2 repolarization. Notably, although both ROS and NO can inhibit mitochondria $(87,281)$, M2 polarizationinhibition has been proposed to be specifically caused by NO, since ROS inhibition by $N$-acetyl-cysteine (NAC) has no effect (353).

Cells are very flexible in relation to the type of substrate used; removal of a particular substrate or blocking a metabolic route is quickly compensated by others, to obtain energy. Metabolic changes imply a metabolic reprogramming due to epigenetic modifications, signal transduction, and transcriptional regulation; changes in metabolite levels also affect cell state. Since metabolic changes occur during $М \Phi$ polarization and metabolic intermediates are not just used to get energy but also directly contribute to drive specific $М \Phi$ phenotypes, targeting these metabolites to polarize $\mathrm{M} \Phi$ and, possibly, to impact on several diseases is an exciting prospect.

Various metabolic strategies are able to redirect $М \Phi$ polarization, for example, enhancing the oxidative metabolism and protecting mitochondria allow M2 reprogramming (239, 240, 353). The metabolic sensor 5' adenosine monophosphateactivated protein kinase (AMPK) activation by metformin and 5-aminoimidazole-4-carboxamide-1-b-4-ribofuranoside (AICAR) with the subsequent oxidative metabolism stimulation leads to M2 polarization also in vivo $(21,46,239,240,399)$ and decreases MФ infiltration into the central nervous system in multiple sclerosis mice models, thus reducing the progression of disease $(239,240)$. Interestingly, miR-33 drives M1 polarization by targeting AMPK (253). By contrast, it has been suggested that metformin inhibits M2 polarization of tumorassociated MФ (TAM), thus preventing cancer metastasis both in vivo and in vitro, in murine and human $\mathrm{M} \Phi(50,71)$. It is conceivable that metformin's effect varies according to the microenvironmental changes $(46,139)$.

The NAD-dependent deacetylases sirtuins also modulate metabolism and impact MФ polarization in vivo $(138,146$, 153, 167, 237). By favoring oxidative metabolism, sirtuins inhibit M1 activation being anti-inflammatory during response against pathogens. In this context, the role of the nicotinamide phosphoribosyl transferase, an enzyme involved in $\mathrm{NAD}^{+}$biosynthesis and sirtuin function, is crucial also in vivo and, possibly, linked to PPAR $\gamma(17,335)$. SIRT3 modulates SDH activity and suppresses ROS by deacetylating and activating MnSOD (55). SIRT1 and SIRT6 favor the M2 phenotype by switching the metabolism from glycolysis to FA $\beta$-oxidation (186) and by inhibiting NF- $\kappa \mathrm{B}$ and STAT3, as observed in human $M \Phi$ cell lines and in primary human and murine MФ (48, 385). Also, SIRT2 and SIRT6 stimulate the anti-inflammatory pathway in murine MФ (175, 188).

Accordingly, the flavonoid quercetin inhibits M1 polarization and inflammation in high fat diet (HFD)-fed mice through an AMPK/SIRT1-mediated mechanism (75), and the phytoalexin resveratrol activates SIRT1 and attenuates the inflammatory response in murine $\mathrm{M} \Phi$ by inhibiting $\mathrm{NF}-\kappa \mathrm{B} /$ ROS-mediated IL-6 secretion along with $\mathrm{TNF} \alpha$ and NO synthesis. Resveratrol promotes M1-to-M2 plasticity and M1 cell death in murine and human $\mathrm{M} \Phi$ by a mechanism involving Arg1 activation (53, 177, 250, 292); however, it has controversially been reported that resveratrol inhibits TAM M2 polarization observed in vivo in mice (323). Synthetic SIRT1-activating compounds have been developed: They have a greater potency compared with resveratrol; among them, SRT1720 has an anti-inflammatory effect that acts by reducing the number of $\mathrm{M} 1$ in mice $(44,48)$. The SIRT3 activator Honokiol inhibits iNOS expression, NF- $\kappa \mathrm{B}$ and $\mathrm{TNF} \alpha$ secretion in LPS-stimulated murine MФ (39).

Another molecule proposed as a metabolic modulator is the dimethylfumarate (DMF; Tecfidera), a fumaric acid methyl ester rapidly hydrolyzed to its active metabolite monomethyl fumarate (MMF). DMF reduced the progression of multiple sclerosis in a phase III clinical trial (110); since fumarate is a TCA cycle metabolite, it might act on MФ metabolism favoring an M2 phenotype. In fact, DMF triggers a cytokine production shift from a pro-inflammatory to an anti-inflammatory pattern, inducing an M1-to-M2 phenotype shift in vitro. In particular, in LPS/IFN $\gamma$-treated human peripheral blood mononuclear cells, the expression of CXCL8, CXCL9, and CXCL10 chemokines is inhibited by DMF; whereas MMF-treated ones upregulate the anti-inflammatory IL-4, IL-5, IL-10, and IL1-ra (248, 335). Although controversial, DMF is categorized as anti-inflammatory by possibly activating the transcription factor Nrf2 inducing antioxidant genes (335). 
Consistent with the earlier considerations, PDK1 plays a key role in in vitro murine MФ polarization (332); it phosphorylates and inhibits some components (e.g., PDH-E1 $\alpha)$ of the PDH complex, converting pyruvate derived from glycolysis to acetyl-CoA. Acetyl-CoA, in turn, enters the Krebs cycle, thus generating NADH and $\mathrm{FADH}_{2}$ fueling the ETC for OxPhos. PDK1 promotes M1 polarization, enhances glycolysis and lactate production, and inhibits M2 (332). Moreover, its downregulation decreases glycolysis and lactate production in response to TLR activation while increasing mitochondrial respiration; it also reduces $I L-6$ and iNOS mRNA levels as well as iNOS and COX-2 protein levels, therefore decreasing the induced M1-inflammatory response (332).

As reported earlier, $\operatorname{PPAR} \gamma$ is crucial for alternative $\mathrm{M} \Phi$ activation $(47,249,258,259)$. Also, PGC- $1 \beta$ promotes M2 polarization through IL-4 and STAT6 in BMDM, while stimulating FAO and mitochondrial biogenesis (357). The energy production coordinator PGC- $1 \alpha$ promotes oxidative metabolism; it is upregulated in M2, and its over-expression stimulates M2 polarization (72).

MФ polarization is also influenced by FA-induced metabolic reprogramming; different FAs exert different effects on МФ polarization, for example, human $М \Phi$ incubated with the saturated FA palmitate, but not with oleate, stimulate inflammatory cytokines and ROS production inducing M1 polarization and reducing OxPhos dependency through ceramidedependent PPAR $\gamma$ inhibition (259). Vice versa, the omega-3 FA eicosapentaenoic acid (EPA) increases IL-10 and reduces INF- $\gamma$ levels, thus promoting an M1-to-M2 shift in mice (33). Similarly, other lipid derivatives such as lipoxins and palmitoleic acid have anti-inflammatory effects (287).

In obesity, adipose tissue is characterized by infiltrating pro-inflammatory $\mathrm{M} \Phi$; high levels of circulating FA might trigger M1 polarization in the adipose tissue of obese individuals. The effect of modulating FAO in the presence of high levels of FA is still unclear; activating FAO in MФ might be beneficial since it metabolizes FA, an antiinflammatory effect. On the other hand, it has been shown that palmitate oxidation fuels OxPhos and produces ROS, thereby activating the inflammasome and pro-inflammatory cytokines production.

As discussed earlier, hypoxia-activated HIF- $1 \alpha$ induces $i N O S$ and $I L-1 \beta$; moreover, the effect of HIF- $1 \alpha$ on glucose uptake potentiates glycolysis and pro-inflammatory activity, as observed in cultured human and murine $\mathrm{M} \Phi$ and in a murine model of atherosclerosis $(143,338)$. More recently, it has been found that HIF-1 $\alpha$ leads to increased mitophagy, which, in turn, induces a metabolic reconfiguration toward glycolysis, causing M1 polarization (85). Notably, intramuscular $\mathrm{pO}_{2}$ is lower compared with atmospheric oxygen levels even in physiological conditions; since $\mathrm{pO}_{2}$ influences cell physiology, data obtained in vitro might not fully reproduce in vivo processes also for this reason, even though M1 and M2 metabolic features occur in different tissue $\mathrm{pO}_{2}$ conditions.

In conclusion, these data emphasize the potentiality of metabolic therapies in directing $\mathrm{M} \Phi$ activation.

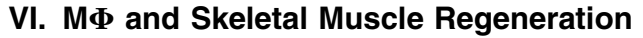

Besides their role in innate immunity, MФ are also involved in tissue repair $(108,180,201)$. In particular, we will hereby describe the crucial role of $\mathrm{M} \Phi$ in allowing efficient skeletal muscle regeneration (245); the muscular regenerative program is first illustrated, followed by the analysis of the role of $\mathrm{M} \Phi$ in regeneration on acute injury and chronic muscle diseases. In particular, the relevance of the M1-to-M2 switch and the interaction between M $\Phi$ subsets, satellite cells (SCs), and other muscle resident or infiltrating cells is discussed.

\section{A. Skeletal muscle regeneration}

In response to skeletal muscle damage, a finely regulated regeneration program is rapidly activated to allow muscle recovery (339). Muscle regeneration relies on proliferation and differentiation of SCs, the muscle resident stem cells localized under myofiber basal lamina usually quiescent during homeostasis $(205,389)$. In response to muscle damage, in both acute and chronic conditions, SCs exit from quiescence and undergo proliferation followed by either asymmetric division-mediated commitment to terminal muscle differentiation or return to quiescence as part of the SC pool (40).

SC asymmetric division guarantees that SC number does not change on multiple regenerative events, thus maintaining the muscles' regenerative potential (374). The ability of SCs to stay in a quiescent state is essential for SC self-renewal and maintenance of muscle stem cells pool $(19,227)$. Quiescence is a regulated condition, characterized by the expression of negative cell cycle regulators such as $\mathrm{p} 27^{\mathrm{Kip} 1}$ and retinoblastoma tumor suppressor protein $(\mathrm{Rb})(36)$.

Several markers for SCs and regenerating myofibers have been identified. Quiescent SCs specifically express Pax7 (306); SC activation is induced by mitogenic factors released in the damaged muscle area, such as insulin-like growth factor-1 (IGF-1), which downregulate $\mathrm{p} 27^{\mathrm{Kip} 1}$, allowing cell cycle entry (37). Activated $\mathrm{SCs}\left(\mathrm{Pax}^{+}, \mathrm{Myf}^{+}\right)$are also characterized by MyoD expression, typical of proliferating myoblasts that are able to undergo both self-renewal and differentiation.

Myoblast commitment to terminal muscle differentiation-involving Wnt and Notch pathways-is characterized by Pax 7 downregulation and induction of early and late muscle regulatory factors (MRFs), specifically Myogenin and MRF4/Myf6 $(25,58)$. At this stage, differentiating myocytes fuse with pre-existing myofibers or with each other, to generate multinucleated myotubes expressing embryonic myosin heavy chain ( $\mathrm{MyH} 3 / \mathrm{eMyHC})$ and undergoing further fusion with neighboring myotubes to produce myofibers (339).

Newly formed myofibers can be distinguished by their small caliber, by eMyHC expression, and by centrally located myonuclei; whereas mature myofibers have bigger diameters, express different isoforms of adult MyHC (Myhl/fastMyHC2x, Myh2/fastMyHC2a, Myh4/fastMyHC2b, Myh7/ slowMyHC1, Myh8/perinatal MyHC) (305) along with other late differentiation markers (e.g., muscle creatine kinase) and their nuclei are peripherally located (Fig. 11) (54, 393). Regenerating myofibers express the same $\mathrm{MyHC}$ isoforms that are characteristic of the specific damaged muscle, in both acute and chronic injury (305).

A detailed histopathological analysis of several regenerative stages on acute injury is shown in Figure 12A, whereas 


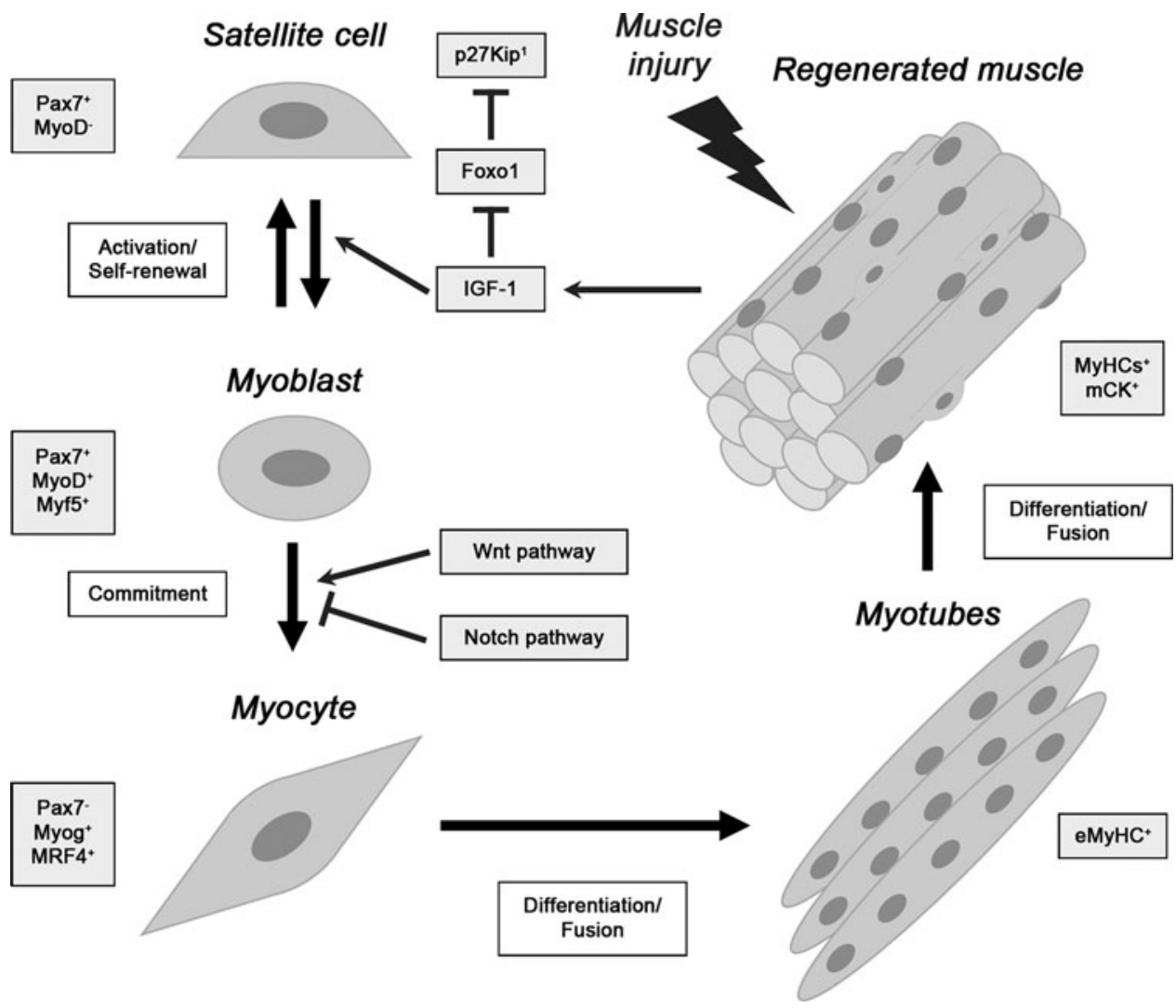

FIG. 11. Muscle regeneration process relies on SCs. During muscle homeostasis, SCs are maintained in a quiescent state by the activity of negative regulators of proliferation. In response to muscle injury, SCs undergo activation, exit from quiescence, and enter the cell cycle while undergoing symmetric or asymmetric divisions. Some daughter cells return to quiescence as part of the SC pool (self-renewal); several daughter cells activate a muscle differentiation program regulated by specific signaling pathways and leading to the expression of both early and late muscle regulatory factors (Myf5, MyoD, Myogenin, Myf6/MRF4). Differentiated myocytes fuse with each other and with pre-existing myofibers to generate new multinucleated myotubes that express embryonic myosin heavy chain $(M y H 3 / \mathrm{eMyHC})$ and undergo further fusion with neighboring myotubes to produce mature myofibers expressing different isoforms of adult MyHC and other markers of terminal differentiated fibers (such asmCK), determining regeneration of damaged muscle areas. eMyHC, embryonic myosin heavy chain; mCK, muscle creatine kinase; MyHC, myosin heavy chain; SCs, satellite cells.

immunofluorescence detection of $\mathrm{M} \Phi$ and newly formed myofibers is shown in Figure 13A. The scheme in Figure 14 shows the timing and trend of $\mathrm{M} \Phi$ recruitment after muscle injury in parallel with the different muscle repair stages in which they are involved (125).

\section{B. Role of $M \Phi$ in skeletal muscle regeneration}

Muscle infiltrating immune cells play a critical role in skeletal muscle regeneration; neutrophils and $\mathrm{M} \Phi$ are very abundant in damaged muscles, as observed in human biopsies and in mice $(6,93,340,342)$, whereas eosinophils (129) and lymphoid cells $\left(\mathrm{CD}^{+}, \mathrm{CD}^{+}\right.$, and regulatory $\mathrm{T}$ cells/Treg) are rare although relevant for successful regeneration $(29,34$, 367). However, a definitive understanding of the complex temporally coordinated $\mathrm{M} \Phi$ roles in acute injury and in muscle degenerative diseases, and of the mechanisms regulating the crucial $\mathrm{M} \Phi$ polarization occurring in regeneration, is still lacking $(158,296,366)$.

As stated earlier, $\mathrm{M} \Phi$ are able to assume, in response to a large variety of stimuli, a wide spectrum of polarization statuses corresponding to defined transcriptome signatures (386). Although the gross in vitro M1/M2 classification, in- cluding M2 subtypes, is schematically useful, it represents a conventional description that does not exhaustively recapitulate the in vivo process and the MФ milieu in regenerating muscle, characterized by cell-to-cell interactions and by several uncharacterized M $\Phi$ phenotypes intermediate between M1 and M2 subtypes (142).

1. On acute injury. After injury, the number of $\mathrm{M \Phi}$ within the skeletal muscle (located in the interstitial space of regenerating muscle) exponentially increases; damaged myofibers undergo necrosis; and release of normally musclecompartmentalized factors recognized by TLRs triggers the production of pro-inflammatory cytokines and chemokines, including CCL2, which recruit bone marrow-derived monocytes $\left(\mathrm{Ly} 6 \mathrm{C}^{+} \mathrm{CCR} 2^{+} \mathrm{CX} 3 \mathrm{CR} 1^{\text {low }}\right.$ ) into damaged muscles. In murine models of muscle injury, monocytes differentiate into highly plastic M $\Phi$ (Figs. 1 and 2) that, in response to cues from a damaged environment, polarize toward $\mathrm{M} 1 / \mathrm{Ly}_{6} \mathrm{C}^{+}$ and $\mathrm{M} 2 / \mathrm{Ly} 6 \mathrm{C}^{-}$phenotypes mounting and resolving the inflammatory response, respectively $(6,224,319)$.

Blocking $М \Phi$ recruitment to damaged muscles in the first $24 \mathrm{~h}$ after injury impairs muscle regeneration, increasing necrotic fibers and fat deposition, as revealed in transgenic 

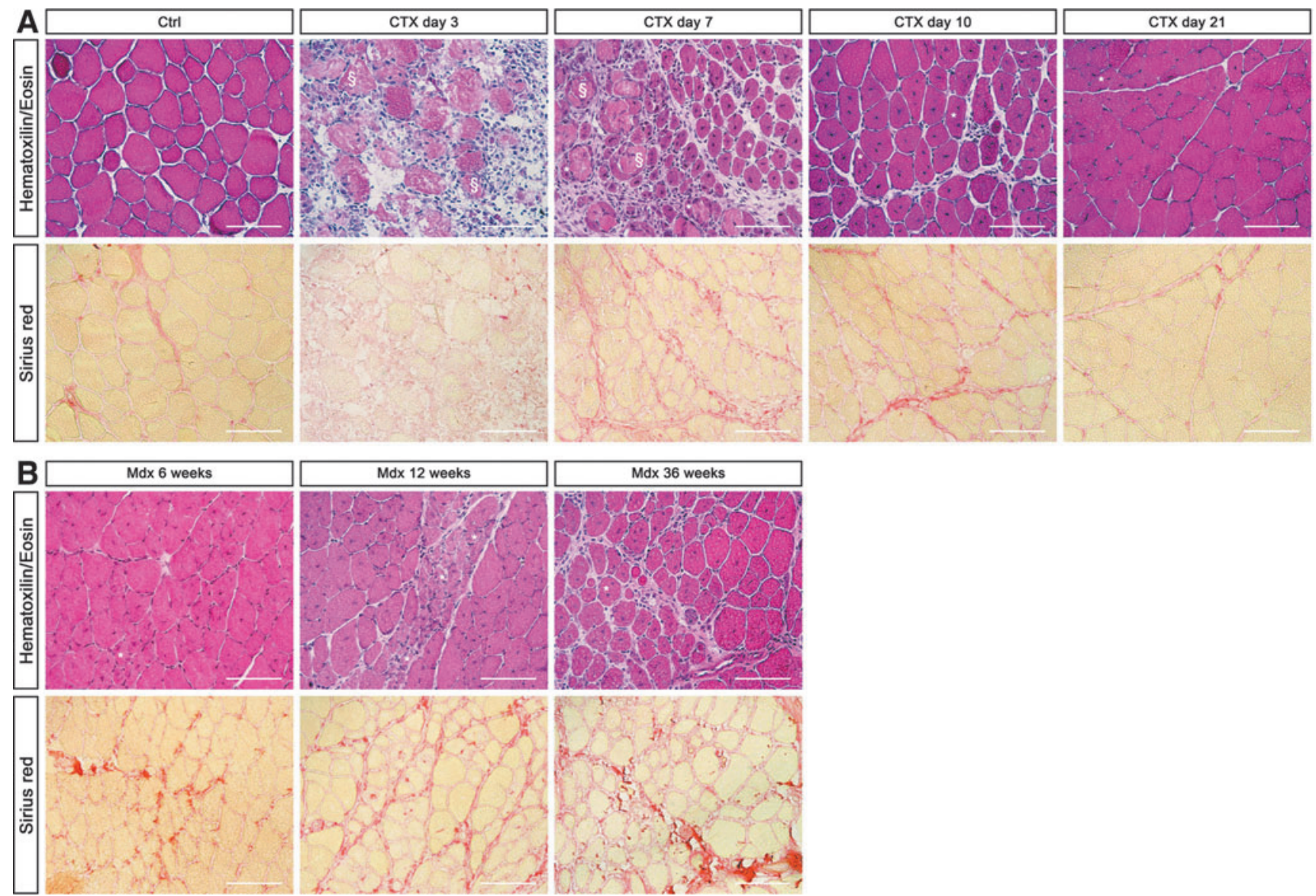

FIG. 12. Histopathological analysis of skeletal muscle regeneration on acute and chronic injury. H/E and Sirius red (specific for collagen isoforms) stainings performed on TA cryosections highlight skeletal muscle architecture and ECM deposition, respectively. (A) Time course of muscle repair on injection of CTX in TA muscles (uninjured control muscleCtrl, CTX day 3, CTX day 7, CTX day 10, and CTX day 21). At early stages of regeneration on acute injury (day 3 after CTX injection), H/E reveals a massive muscle infiltrate along with necrotic fibers (§) in the damaged area. The intermediate stage of muscle regeneration (day 7 on CTX injection) is characterized by huge ECM deposition (Sirius red staining) and small regenerating centro-nucleated myofibers $(*)$, indicating an engagement in the muscle regeneration process. At a more advanced stage of CTX-induced regeneration (day-10), the majority of regenerating myofibers are bigger (due to increased size) although still centro-nucleated, whereas interstitial infiltrate and collagen deposition are strongly reduced and, eventually, completely disappear at a late stage of regeneration (day 21 on damage) when regeneration appears almost completed and is mainly characterized by mature myofibers with peripherally located nuclei just beneath the sarcolemma, even though few regenerating centro-nucleated fibers are still detectable. On an acute injury, inflammatory infiltration and collagen deposition are transient and the regenerative process culminates in a complete regeneration of the damaged area with an overall recovery of muscle architecture, reduced interstitial space, and no detectable MPs and regenerating myofibers. Scale bar $=200 \mu \mathrm{m}$. (B) Muscle regeneration of dystrophic TA muscle from mdx mice at different stages of disease: 6,12 , and 36 weeks, corresponding to necrotic/inflammatory, regenerative, and fibrotic phase of dystrophic pathology, respectively. Young mdx mice (6 weeks old) show several centro-nucleated regenerating fibers, low inflammatory infiltrate, and collagen deposition that are necessary to support muscle regeneration and proper compensatory recovery of muscle. TA muscles of 12-week-old mdx mice (corresponding to the regenerative phase of DMD) are quite comparable to day 7 after CTX-induced damage (A), in terms of centrally nucleated fibers, inflammatory infiltrate, and ECM deposition. Differently from acute injury, which culminates in a complete regeneration of the damaged muscle (A), at the late stage of chronic disease (36-week-old mdx mice), the muscle architecture appears more damaged, with persistent inflammatory infiltration, reduced although still detectable and regenerating centro-nucleated fibers and irreversible accumulation of collagen, leading to increased fibrosis. Scale bar $=200 \mu \mathrm{m}$. CTX, cardiotoxin; DMD, Duchenne muscular dystrophy; ECM, extracellular matrix; H/E, hematoxilin/eosin; TA, tibialis anterior. Color images are available online.

mice expressing the diphtheria toxin receptor (DTR) under the $C D 11 b$ promoter control (CD11b-DTR mice), so that $\mathrm{CD} 11 \mathrm{~b}^{+}$cells (mainly $\mathrm{M} \Phi$ ) might be depleted on diphtheria toxin injection $(6,374)$. Moreover, studies in mice highlighted the crucial role of $\mathrm{M} \Phi$ infiltration in muscle regen- eration, which is impaired by genetic deletion of CCR2 (CCL2 receptor) or CCL2, both causing lower MФ recruitment $(191,197,311,376)$.

Experiments in mice suggest that monocyte recruitment occurs as a result of an early activation of resident $\mathrm{M} \Phi$ 

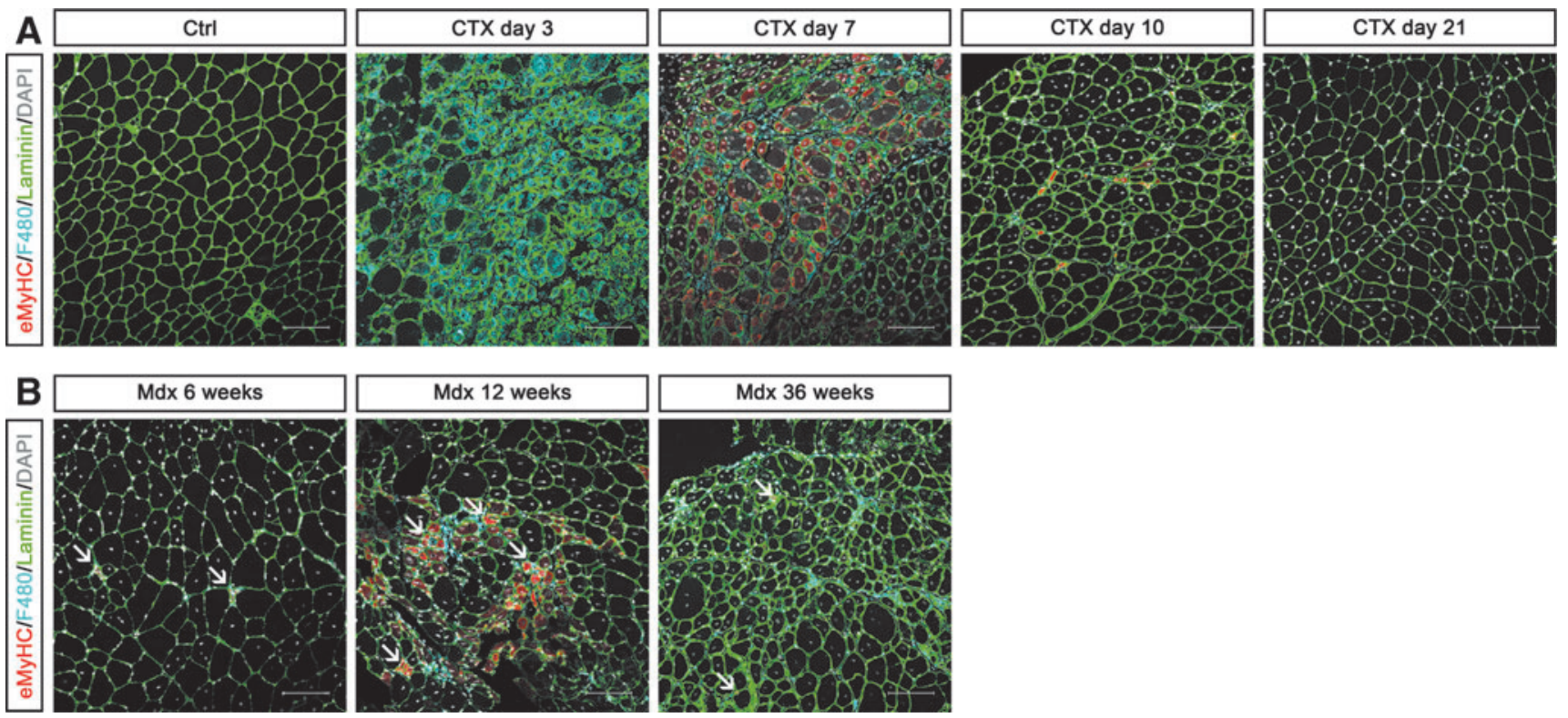

FIG. 13. Regenerating myofibers and MP infiltration on acute and chronic injury. Immunofluorescence staining of TA muscle cryosections (similar to those used for Fig. 12), using antibodies recognizing eMyHC as a marker of newly formed myofibers (red) Laminin (green) to label myofiber perimeter and F4/80 (cyan) as a surface marker of MPs. Nuclei were stained with DAPI (white). Scale bar $=100 \mu \mathrm{m}$. (A) Time course of CTX-injected TA (uninjured control muscle-Ctrl, CTX day 3, CTX day 7, CTX day 10, and CTX day 21). At an early phase of regeneration after CTX injection (day 3), a massive inflammatory infiltrate is detectable and is mainly represented by F4/80 ${ }^{+}$MPs (cyan) (see also day 7). At an intermediate phase of regeneration (CTX day 7), several newly formed myofibers can be readily distinguished by their small caliber, by a huge expression of eMyHC (red), and by their centrally located myonuclei. These markers almost completely disappear at a later stage of regeneration (day 10) and become undetectable later on (day 21), where some fibers show peripherally located nuclei. (B) Muscle cryosections of TA from mdx mice at different stages of disease: 6, 12, and 36 weeks, corresponding to necrotic/inflammatory, regenerative, and fibrotic phase of dystrophic pathology, respectively. The early stage of pathology is characterized by low level of eMyHC ${ }^{+}$regenerating fibers (red) (arrows), several centronucleated regenerating fibers, and low level of F4/80 ${ }^{+} \mathrm{M} \Phi$ (cyan). In 12-week-old mdx mice, regenerating fibers (characterized by huge expression of eMyHC) and massive $\mathrm{M} \Phi$ infiltration (F4/80 cells) are abundant in dystrophic muscles and are similar to injured muscles at the intermediate phase of regeneration (day-7 CTX injection). Dystrophic muscles are characterized by chronic inflammation, and, indeed, MФ are still abundant in 36-week-old mice and muscle regeneration is reduced but still detectable (some $\mathrm{eMyHC}^{+}$fibers and centro-nucleated but small myofibers). Color images are available online.

$\left(\mathrm{Ly} 6 \mathrm{C}^{+} \mathrm{CX} 3 \mathrm{CR}^{-}\right)$releasing chemoattractants $\mathrm{CXCL} 1$ and CCL2, determining a massive neutrophil influx, in turn responsible for $\mathrm{Ly} 6 \mathrm{C}^{+} \mathrm{CCR} 2^{+} \mathrm{CX} 3 \mathrm{CR} 1^{\text {low }}$ circulating monocytes extravasation toward damaged skeletal muscle $(26$, 340). The involvement of muscle-resident $M \Phi$ in regeneration is still obscure; transplantation of WT DT-insensitive bone marrow cells in $C D 11 b$-DTR mice-used to deplete $\mathrm{M} \Phi$ and to discriminate between muscle-resident and recruited $\mathrm{M} \Phi$ - demonstrated that muscle-resident $\mathrm{M} \Phi$ are involved in monocyte recruitment after muscle damage (42).

On the other hand, if $M \Phi$ recruitment to the muscle is blocked (for example in $C C R 2^{-1-}$ mice), the muscle fails to regenerate, suggesting that resident $M \Phi$ are only poorly involved in regeneration $(60,376)$, possibly due to the low number of $М \Phi$ residing in skeletal muscles in physiological conditions (6).

Besides neutrophils, $\mathrm{T}$ cells have also been implicated in monocyte/M $\Phi$ recruitment via the CCL2/CCR2 axis; they contribute to CCL2 production and proper regeneration (394). Moreover, Treg rapidly accumulates in acutely injured and dystrophic muscles, ameliorating muscle repair by suppressing inflammation and enhancing SC expansion (29, 34, 367). a. M1-to-M2 switch in muscle regeneration. The early steps of muscle regeneration are associated with pro-inflammatory M1 deriving from recruited monocytes ( $\mathrm{Ly} 6 \mathrm{C}^{+} \mathrm{CCR} 2^{+} \mathrm{CX} 3 \mathrm{CR} 1^{\text {low }}$ ), whereas later on, the intermediate-late steps of regeneration, in mice, are associated with $\mathrm{Ly} 6 \mathrm{C}^{-} \mathrm{CX} 3 \mathrm{CR} 1^{\text {high }}$ anti-inflammatory M2. M1 promote phagocytosis of necrotic fibers and debris, sustain inflammation, and support activation and proliferation of SCs during early regeneration; whereas M2 promote the resolution of inflammation and enhance muscle regeneration by inducing muscle progenitor cells (MPCs) differentiation (194, 291).

Recently, a gene expression analysis on murine $\mathrm{M} \Phi$, sorted as GFP-positive cells from CX3CR $1^{\mathrm{GFP} /+}$ mice at four subsequent time points after cardiotoxin (CTX)-induced muscle damage, revealed highly dynamic changes in $\mathrm{M} \Phi$ expression profile in response to injury (356) at different steps of regeneration: (i) $\mathrm{Ly} 6 \mathrm{C}^{+}-\mathrm{M} \Phi$ show an $\mathrm{IFN} \gamma$-independent inflammatory profile; (ii) a glycolytic-to-oxidative metabolism switch sustains the anti-inflammatory M2 polarization; (iii) Ly6C ${ }^{-}$-proliferating $\mathrm{M} \Phi$ highly express $\mathrm{M}-\mathrm{CSF}$, and the administration of anti-M-CSF-receptor antibody during muscle regeneration suppresses $\mathrm{M} \Phi$ - but not neutrophil- 


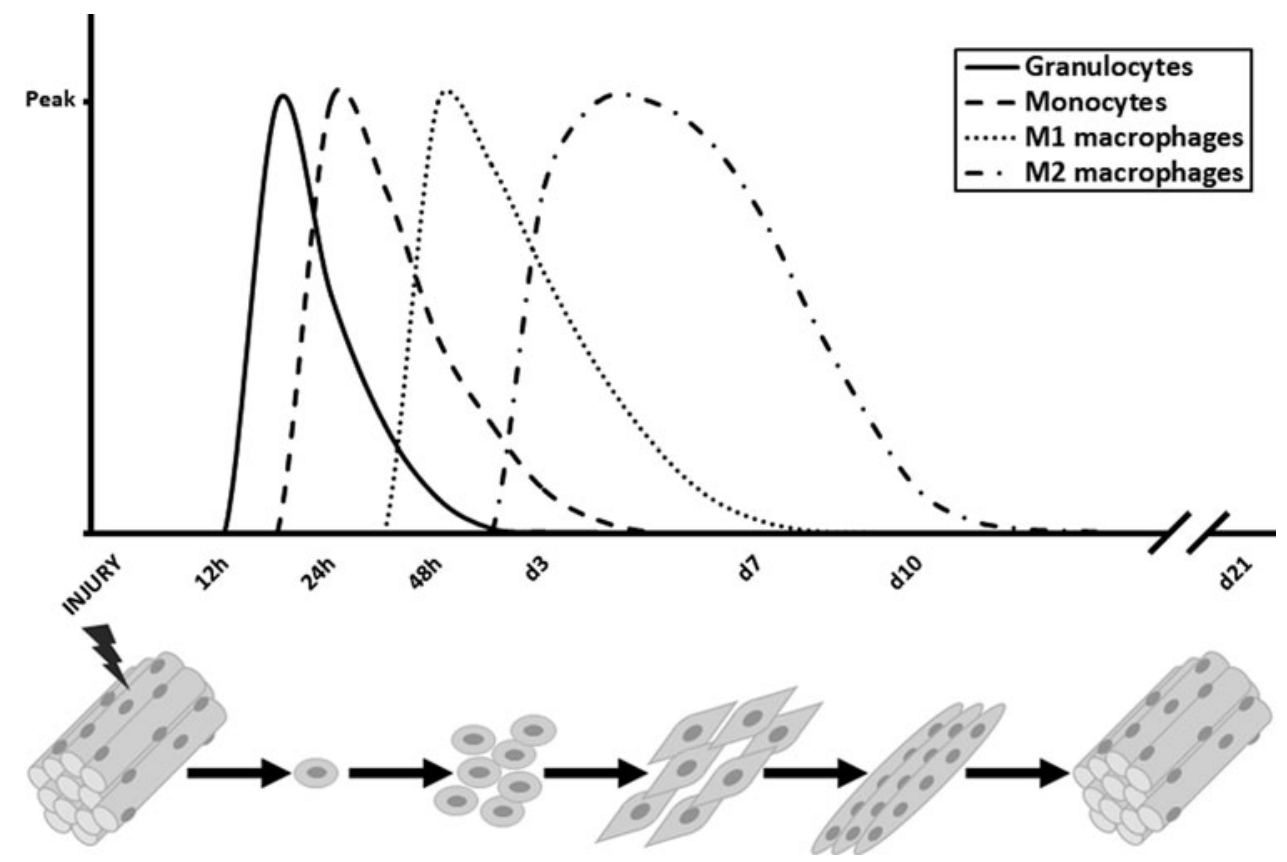

FIG. 14. Timeline of recruitment and activity of immune cells during skeletal muscle regeneration on acute injury. Immune cells are sequentially recruited during the regenerative response, starting from the pro-inflammatory phase, characterized by cell debris removal and activation of SCs and other resident cells, and culminating with activation of the anti-inflammatory program that sustains MPC differentiation and recovery of muscle architecture and function. In vivo experiments demonstrated that granulocytes, in particular neutrophils, are rapidly recruited and infiltrate the damaged area in a few hours, reaching the peak between 12 and $24 \mathrm{~h}$ from the event of injury. They are mainly responsible for maintenance of inflammation and recruitment of other immune cells. Later (between 1 and 2 days postinjury), monocytes reach the injury site and differentiate toward pro-inflammatory M1 MФ (2/3 days postinjury) that are responsible for removal of cell debris and stimulation of SCs proliferation (in vitro experiments). M1 are followed by anti-inflammatory/ pro-myogenic M2 (between 3 and 7 days postinjury), supporting SCs differentiation (in vitro experiments). To note, the graph is a schematic representation typical of CTX-injury; however, it does not take into account the specific timeline variability, which, indeed, is associated to different injury signals and also to different concentrations of the same damage stimulus (125). MPC, muscle progenitor cell.

infiltration and compromises muscle repair, affecting SC proliferation and differentiation and increasing fibrosis (307); and (iv) Ly6C ${ }^{-}-\mathrm{M} \Phi$ produce secretory ECM-related molecules involved in intercellular communication and regeneration, such as matrix metalloproteinase-2 (MMP2).

A sequentially synchronized recruitment and activity of different $\mathrm{M} \Phi$ subtypes, with an M1-to-M2 shift, is required for successful muscle regeneration $(6,372)$; in fact, chronic M1 activation exacerbates muscle injury by releasing mediators of cell damage and cytokines amplifying inflammation. For this reason, in parallel or soon after any inflammatory response, mechanisms aimed at reducing inflammation are activated; M2 anti-inflammatory cytokines, such as IL-10, inactivate pro-inflammatory genes and further drive MФalternative activation (364).

The transition from the early M1 immune response to the intermediate-late M2 immune response is, therefore, required and, functionally, corresponds to the progression of the myogenic regenerative program, since M2 stimulate myogenic differentiation and promote myocyte fusion (297). Interference with M2 polarization is detrimental on muscle regeneration, and in vitro polarized $\mathrm{M} \Phi$ and FACS-isolated Ly6C $-\mathrm{M} \Phi$ are beneficial when transplanted into injured skeletal muscle $(294,344,372)$.

However, the cause of the sequential presence of M1 and M2 in the damaged area of skeletal muscle is still a matter of debate; it is not clear whether skeletal muscle M1 are able to repolarize to $\mathrm{M} 2$, due to changes in the damaged microenvironment, or whether new $\mathrm{Ly} 6 \mathrm{C}^{-} \mathrm{CCR} 2^{-} \mathrm{CX} 3 \mathrm{CR} 1^{+}$monocytes are recruited, at later stages, to a repair-promoting environment (e.g., when CX3CL1/fractalkine attracting M2 is produced by resident $\mathrm{M} \Phi$ due to interaction with dying cells) where they differentiate to $\mathrm{M} 2$, as further suggested by selective depletion of Ly6C ${ }^{-}$monocytes enhancing M1 generation $(102,103,116,121)$.

By contrast, a pivotal paper, first describing the M1-to-M2 polarization within muscle as dependent on necrotic muscle phagocytosis, supports the hypothesis of $\mathrm{M} \Phi$ phenotypic transition within muscle by which injured skeletal muscle recruits only pro-inflammatory monocytes $\left(\mathrm{Ly}_{6 \mathrm{C}}{ }^{+} \mathrm{CCR} 2^{+}\right.$ $\mathrm{CX} 3 \mathrm{CR} 1^{\text {low }}$ ) that, within muscle, switch phenotype to become proliferating anti-inflammatory $\mathrm{Ly}_{6 \mathrm{C}}^{-} \mathrm{CCR} 2^{+} \mathrm{CX} 3$ $\mathrm{CR} 1^{\text {high }}$ cells, further differentiating into $\mathrm{F} 4 / 80^{+} \mathrm{M \Phi}(6)$. Moreover, the MAPK-phosphatase-1 (MKP-1)-p38-Akt axis is crucial for sequential M1-to-M2 transition, and deregulation of $М \Phi$ skewing by MKP-1-loss, in mice, results in impaired SC activity and defective regeneration (268).

Also, AMPK $\alpha 1$ is crucial for phagocytosis-induced pro-toanti-inflammatory $\mathrm{M} \Phi$ skewing, at the time of inflammatory resolution; indeed, damaged muscles of $A M P K \alpha 1^{-/-}$mice show decreased M2 and increased M1 subsets, along with regenerative impairment (226). In addition, CCAAT 
enhancer-binding protein $(\mathrm{C} / \mathrm{EBP}) \beta$ in infiltrating $\mathrm{M} \Phi$ is required for the upregulation of $\mathrm{M} 2$-specific genes and for M1-to-M2 switch and muscle regeneration (210, 291). The critical role of the $\mathrm{M} \Phi$-phenotypic switch for regeneration is also confirmed by the recent finding that miRNA-155 expression in myeloid cells modulates $M \Phi$ activation in vivo in mice and is necessary for proper muscle regeneration; during the initial inflammatory phase, miRNA-155 suppresses SOCS1 (a negative regulator of the JAK/STAT pathway) (243).

Also, in human skeletal muscle regeneration, different $\mathrm{M} \Phi$ subsets modulate MPC fate; specifically, pro-inflammatory M1 are mainly associated with regenerating areas containing proliferating myogenic cells, whereas M2 are preferentially associated with differentiating myogenic cells (297). Among the different anti-inflammatory M2 subtypes, M2a are associated with halted muscle regeneration, collagen accumulation, fibrogenesis, and angiogenesis, preceded by anti-inflammatory M2c involved in tissue remodeling and regeneration, ECM deposition, and immunoregulation. As reported earlier, under the effect of IL-10, M2c express receptors for pro-inflammatory chemokines, which would possibly serve as a scavenger receptor system to dampen inflammation (270).

2. In chronic muscle diseases: for example, Duchenne muscular dystrophy. Similar to acute injury, also at early stages of neuromuscular disorders, such as Duchenne muscular dystrophy (DMD), muscle regeneration compensates chronic degeneration. However, differently from acute injury, chronic muscle diseases are characterized by overlapping and asynchronous cycles of degeneration and regeneration, associated with continuous $M \Phi$ infiltration producing, at each stage of disease, a mixed $М \Phi$ population containing variable levels of $\mathrm{M} \Phi$ subtypes. The balance between different $\mathrm{M} \Phi$ subpopulations is determinant for the progression of dystrophy in mdx mice, the commonly used mouse model of DMD disease (364).

For instance, the competition between iNOS-producing M1 and Arg1-producing M2a influences the extent of myofiber lysis by МФ. In fact, M2a reduces the cytolytic activity of M1 (363-365). Both M1 and M2a are present in muscles of 4-week-old mdx mice, the acute necrotic stage of the pathology (364). Arg1 expression in M2 increases in mdx muscles as dystrophy proceeds, and Arg1 metabolism might contribute to fibrosis driven by M2 in response to Th2 cytokines; indeed, ablation of Arg1 reduces fibrosis (377).

In DMD patients, the dystrophic pathology onset coincides with the onset of muscle inflammation, suggesting a key detrimental role of inflammatory cells in the progression of DMD and other skeletal muscle degenerative diseases characterized by chronic inflammation (366). The inflammatory progression in DMD patients is similar to what is observed in mdx mice. To study the role of $M \Phi$ in DMD, we recently generated an $М \Phi$-depleted dystrophic mouse model by crossing the CD11b-DTR mouse with the mdx one, and we have confirmed the crucial beneficial role of $\mathrm{M} \Phi$ in muscular dystrophies (unpublished observations).

A detailed histopathological analysis of various stages of skeletal muscle regeneration on chronic injury is shown in Figure 12B. Specific markers, identified by immunofluorescence, show that 12 -week-old mdx mice muscles are similar to CTX-injured muscles at the intermediate phase of regeneration (day-7 CTX injection) (Fig. 13A, B) [reviewed in Rigamonti et al. (282)].

\section{Cross-talk between metabolically diverse $M \Phi$ subpopulations and muscle resident cells}

MФ affect myogenesis and muscle fibrosis, after acute damage and in chronic diseases, by producing cytokines, chemokines, soluble factors, and cross-talking with muscle resident cells, for example, fibroadipogenic progenitor cells (FAPs), fibroblasts, SCs, and endothelial cells $(218,275)$. A reciprocal cross-talk between muscle cells and infiltrating immune cells also occurs, with muscle cells responsible for modulating the MФ phenotypic shift during repair (261). The proper interplay among different cell types ensures phagocytosis of necrotic myofibers, activation of SCs, execution of the myogenic program (6), angiogenesis (247), and SC self-renewal to restore stem cell pool (225), thus forming new functional myofibers and allowing muscle homeostasis.

МФ participate in the final stages of skeletal muscle repair by releasing not only anti-inflammatory mediators, such as TGF- $\beta$ and IL-10, but also pro-regenerative and proangiogenic growth factors such as IGF-1 and VEGF-A, fibroblast growth factor (FGF), placental growth factor, and hepatocyte growth factor (HGF). Also, SDF-1 is necessary for collagen deposition into the damaged muscle and for regeneration (287), and through polyamine and collagen synthesis M2-produced ornithine promotes cell proliferation, fibrosis, and tissue remodeling (272). МФ-produced growth factors act on the ECM, triggering angiogenesis; $М \Phi$ also release MMP2 and urokinase-type plasminogen involved in ECM protein degradation and proteolytic activation of HGF [reviewed in Rybalko et al. (294)], whereas collagen VI is also critical for MФ migration and M2 polarization in mice (47).

Apoptotic cell clearance also contributes to triggering M2 activation and wound healing. Moreover, in response to sterile injury, vessel-associated stem cells (mesoangioblasts) specifically upregulate genes involved in the clearance of apoptotic cells and in M2 polarization-such as CD163-in MФ (23). Notably, MФ-secreted complement $\mathrm{C} 1 \mathrm{q}$ impairs regeneration; blocking the angiotensin-II type 1 receptor by irbesartan induces $\mathrm{M} 2$ polarization and reduces $\mathrm{M} \Phi$-associated $\mathrm{C} 1 \mathrm{q}$ expression, thus ameliorating regeneration and reducing fibrosis (387).

Immune system-produced ROS also participates in tissue repair key signaling and could be beneficial or detrimental depending on their levels. For example, ROS modulate the release of pro-inflammatory cytokines by modulating the balance between reduced and oxidized HMGB1 forms, involved in SC activation and differentiation in damaged skeletal muscle (358). However, excessive ROS levels can hyper-activate the inflammatory response, thus impairing regeneration. It has been suggested that MФ-derived ROS are not involved in skeletal muscle regeneration (171); vice versa, iNOS seems to be crucial in acute muscle damage in vivo, indeed NO stimulates muscle repair (59), and M2 express factors such as SOD1 and thioredoxin that are implicated in HMGB1 reduction (360). A comprehensive analysis of redox regulation in resident and infiltrating cells during skeletal muscle regeneration is reviewed in Le Moal et al. (172). 
FIG. 15. Cross-talk between resident and infiltrating cells during skeletal muscle regeneration. Graphical schematization of a transversal section of skeletal muscle. Several resident and infiltrating cells support regenerative myogenesis; only SCs in their sub-laminar localization, and FAPs and MФ in the interstitial area between muscle fibers are represented. Gray arrows indicate cell interplay and define the directionality of interactions among these three cell populations. FAPs, fibroadipogenic progenitor cells.

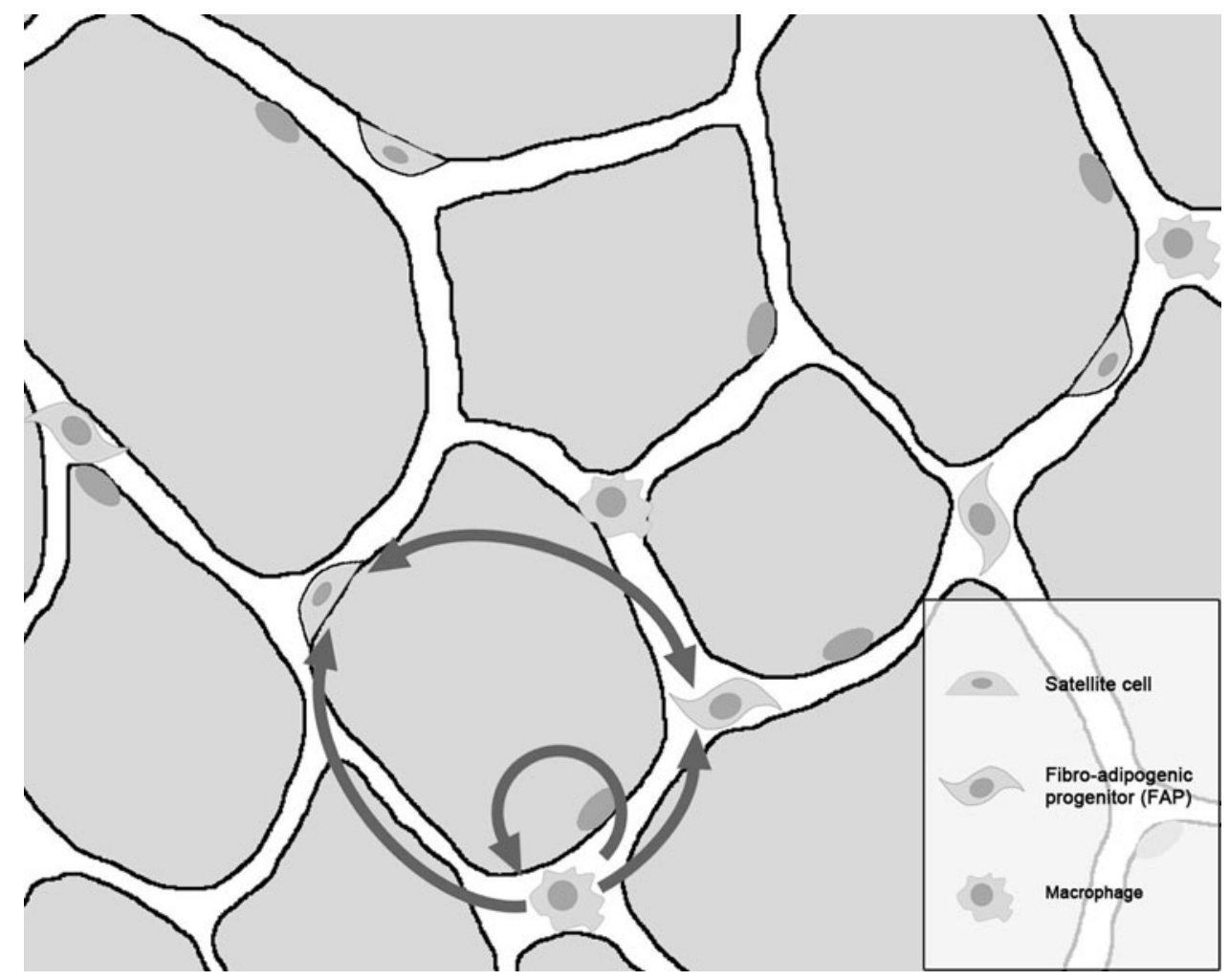

The cross-talk between MФ and SCs and between MФ and FAPs will be discussed (Fig. 15).

1. Cross-talk MФ: SCs. At early stages of regeneration, the cross-talk between SCs and M $\Phi$ is mainly modulated by M1-produced pro-inflammatory cytokines, which further recruit immune cells that are responsible for fiber debris and necrotic cell phagocytosis regulating, in an autocrine manner, the balance between MФ subpopulations. Proinflammatory mediators also exert paracrine effects on muscle cells, for example, activating quiescent SCs that start proliferating, as observed in mice and in humans, and regulating FAP fate $(43,297,343)$. In fact, SCs/MPCs proliferation was found to be associated with M1, both in vivo and in vitro; M1 migrate toward SCs/MPCs, where they stimulate their proliferation and prevent their premature differentiation (Fig. 16) (297).

As described in mice, after the phagocytic phase, an M1to-M2 switch occurs, with M2 inhibiting M1 and reducing pro-inflammatory cytokines-mediated MPCs proliferation while stimulating their differentiation (70).

IFN $\gamma$ rapidly increases in injured muscle; it binds to its receptor expressed by both M1 and SCs/MPCs and activates target genes via JAK-STAT1 $(49,190,222)$. As observed in mice, IFN $\gamma$ promotes M1 polarization and represses SCs/ MPCs differentiation by inhibiting myogenic genes- for example, Myogenin - through recruitment of Jarid2 and polycomb repressive complex-2 to their promoters $(190,381)$. Early in regeneration, IFN $\gamma$ signaling maintains the M1 phenotype, allowing MPCs expansion; however, at intermediatelate stages of regeneration, IFN $\gamma$ signaling must be switched off to avoid terminal muscle differentiation impairment (Fig. 16) (190). IFN $\gamma$ ablation in $\mathrm{mdx}$ mice modulates the M1/M2 balance, this having different consequences at the inflammatory (3-4 weeks of age) and regenerative stages (6-12 weeks of age) of disease. The early inflammatory stage was not affected by $I F N \gamma$ ablation, suggesting that M1 polarization is not strictly dependent on IFN $\gamma$.

By contrast, at the regenerative stage, IFN $\gamma$ deletion is beneficial in that it promotes pro-regenerative M2 polarization and increases MyoD expression (363). Similar to IFN $\gamma$, TNF $\alpha$ acts both on MФ, promoting M1 polarization, and on MPCs, affecting regeneration (Fig. 4). TNF $\alpha$ induces transcriptional repression of specific muscle genes (Pax7, MyoD, Myogenin, and MEF2C) in MPCs by Ezh2 recruitment on promoters $(1,58,255)$. IL- 6 , TNF $\alpha$, IL- $1 \beta$, and G-CSF are all known to enhance myogenic proliferation $(41,122)$.

The anti-inflammatory cytokine IL-10 is crucial for the M1-to-M2 switch, improving muscle regeneration in both acutely injured and dystrophic muscles (Fig. 4) (70, 365). IL10 effect on M2 polarization depends on IL-10-mediated AMPK activation, critical for the glycolytic (predominant in M1) to the oxidative metabolism (predominant in M2) switch, as observed in vitro (399). Indeed, $A M P K \alpha 1$ deletion in myeloid cells delays muscle repair, likely by influencing MФ polarization (226). Although demonstrated only in vitro, IL-10-expressing M2 might also promote myoblast proliferation (70).

Moreover, M2-dependent IL-10 production is essential to support mesoangioblast survival and function in vivo (24). IL-4, mainly produced by eosinophils and Th2 cells, contributes to promoting M2 polarization, thus creating a proregenerative environment (Fig. 16). IL-4 is also expressed by muscle cells and controls myoblast/myotube fusion; it is secreted by myotubes and binds to myoblast-expressed IL-4R, therefore recruiting myoblasts to myotubes. $I L-4$ is regulated 


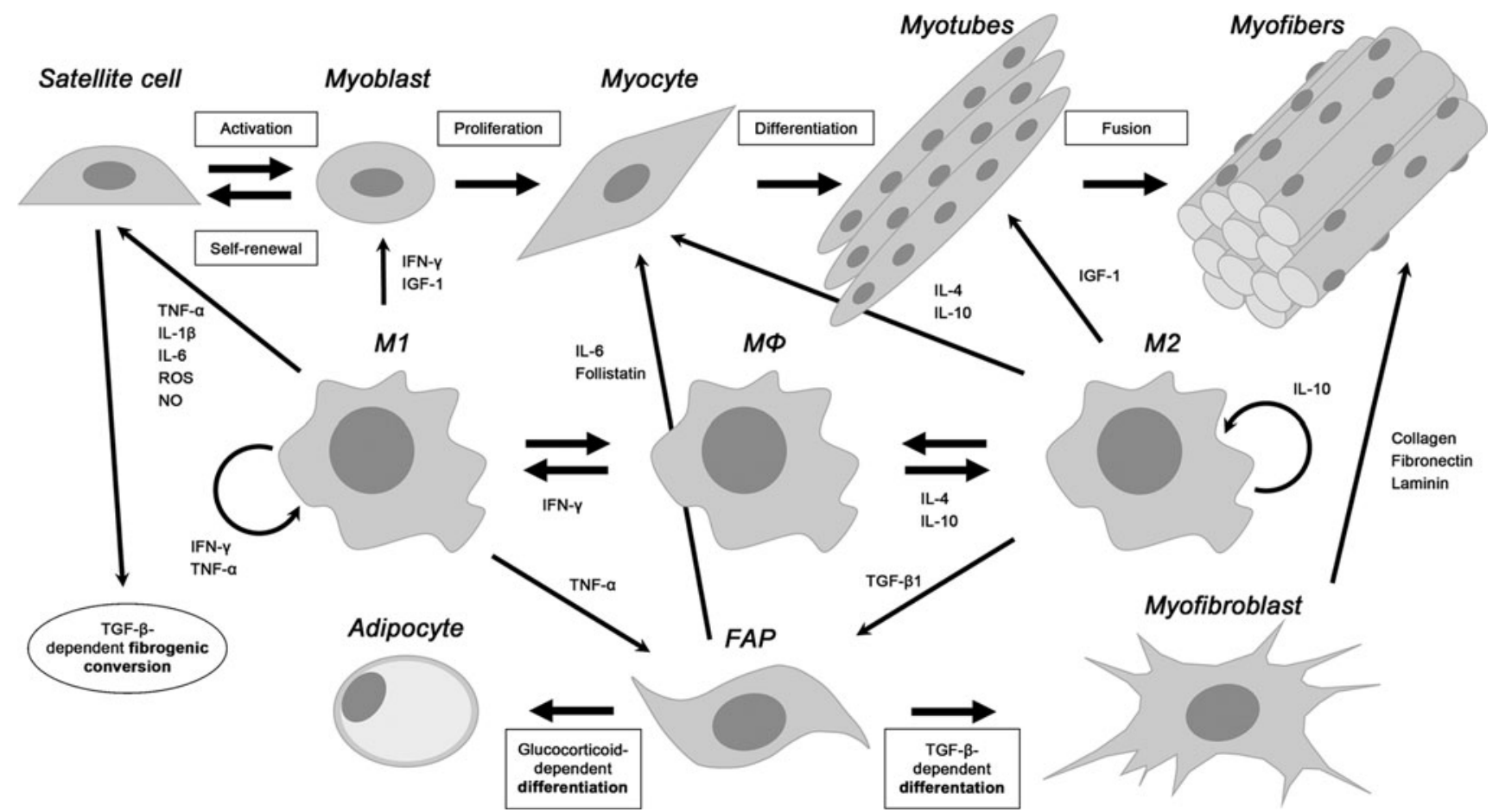

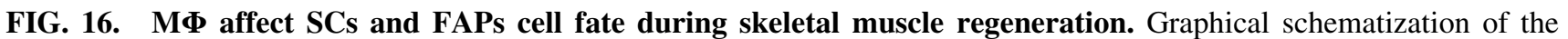
cross-talk through soluble factors (chemokines, cytokines, growth factors, small molecules, or structural proteins) between SCs, FAPs, and MФ. The upper part describes the activation of SCs in response to damage signals: SCs exit from quiescence and undergo a proliferative phase followed by commitment into terminal muscle differentiation, or they return to quiescence to maintain the stem cell pool. Differentiating myocytes fuse with pre-existing myofibers or with each other to originate new multinucleated myotubes that further fuse with neighboring myotubes to produce mature myofibers. A

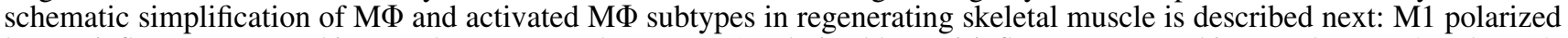
by pro-inflammatory cytokines such as IFN $\gamma$ and TNF $\alpha$; M2 polarized by anti-inflammatory cytokines such as IL-4 and IL-10. M1 produce soluble factors, such as IFN $\gamma, \mathrm{TNF} \alpha$, IL-1, IL-6, NO, and ROS, that contribute to SCs activation and proliferation. Factors derived from M2 MФ (such as IL-4, IL-10, IGF-1) mainly influence myocyte differentiation. MФ also affect FAPs cell fate, represented in the lower part, modulating the balance between FAPs, apoptosis, and differentiation toward fibrogenic or adipogenic lineage. On the other hand, FAPs support SCs differentiation during physiological skeletal muscle regeneration by transient deposition of ECM. In pathological conditions, such as muscular dystrophies, the persistence of activated FAPs in the damaged muscle area has been associated with increased and irreversible production of collagen, replacement of muscle fibers with fibrotic scars, and fat deposition leading to an exacerbation of the degenerative muscle phenotype. Thick arrows represent the progression of cell differentiation or polarization; thin arrows represent the soluble factors (cytokines, chemokines, growth factors, small molecules, or structural proteins) affecting the fate of target cells.

by the transcription factor NFATc2, crucial for myoblast fusion (132).

Produced by several resident cell types or ones infiltrating the regenerative muscle, TGF- $\beta$, crucial for muscle regeneration, is abundant in acutely injured muscles and in muscles of mdx mice and DMD patients [reviewed in Duffield et al. (78)]. In dystrophic muscles, anti-inflammatory TGF- $\beta$ is mostly produced by M2 $(273,398)$. M2-secreted TGF- $\beta$ supports the formation of myotubes (297). However, excessive levels of TGF- $\beta 2$, induced by elevated canonical Wnt signaling in dystrophic muscles, affect SC fate, which undergoes fibrogenic conversion (Fig. 16) (18). TGF- $\beta 1$ can cause fibrosis and neutralizing it significantly promotes muscle regeneration, enhances angiogenesis, prolongs SC activation, and recruits a greater number of M2. If M $\Phi$ infiltration is compromised, endothelial-derived progenitors undergo an endothelial-to-mesenchymal transition, possibly triggered by TGF- $\beta$, collagen accumulates, and the muscle is replaced by fibrotic tissue (400).
In regenerating muscle, both $\mathrm{M} 1$ and $\mathrm{M} 2$ produce IGF-1, although it is more prominently expressed by M2 (191). IGF-1 is a potent regeneration enhancer and is upregulated during the inflammation-to-repair transition phase $(12,235)$. МФderived IGF-1 influences muscle regeneration by a double action: by acting on myogenesis, increasing MPCs proliferation and boosting their terminal differentiation, and in an autocrine manner, by inducing a pro-regenerative M2 polarization and contributing to resolve inflammation (346). Indeed, IGF-1 deletion in myeloid cells impairs M2 accumulation, thus compromising in vivo regeneration (346).

By means of released factors such as IGF-1, MФ also act on intra-myocellular processes that are crucial during regeneration, such as protein synthesis; IGF-1 promotes myofiber protein synthesis predominantly by activating the phosphoinositide 3-kinase (PI3K)/Akt/mTOR pathway and phosphorylating 4E-BP1 and S6K1 $(12,235)$. Notably, the PI3K/Akt/mTOR pathway also mediates autophagy, which might, therefore, in principle, be modulated by $\mathrm{M} \Phi$-produced 
IGF-1 (286). IGF-1 is also produced by muscle cells on exercise; it might have a trophic action, possibly even physiologically, independently from the occurrence of inflammatory conditions (265). Also, IL-6 might act on the Akt/mTOR pathway, influencing protein synthesis and, presumably, autophagy; however, controversial results suggest opposite roles for IL-6 in the regulation of muscle fiber size and regeneration (10).

Notably, after muscle injury, МФ secrete the metalloproteinase Adamts1, which targets and impairs Notch signaling, thus strongly increasing SC activation (77). Moreover, in mice, damaged myofibers and infiltrating $\mathrm{M} \Phi$ release the protein $\mathrm{S100B}$, which expands the myoblast population and promotes M2 polarization modulating collagen deposition. However, prolonged high levels of S100B compromise regeneration by delaying the M1-to-M2 transition and promoting fibrotic tissue deposition (283). Interestingly, anti-inflammatory M2-expressed CD163 is a receptor and scavenger for the cytokine TNF-like weak inducer of apoptosis (TWEAK). A soluble portion of CD163 functions as a decoy receptor for TWEAK, regulating its ability to activate Notch signaling and stimulate MPCs proliferation and tissue regeneration (3).

SCs recruit monocytes/M $\Phi$ using various chemotactic systems that are also useful to reduce apoptosis [reviewed by Rybalko et al. (294)]. The chemotactic factor CX3CL1 is produced by resident $\mathrm{M} \Phi$ on interaction with dying cells and is also expressed by myoblasts; CX3CL1 attracts M2 and also induces the expression of pro-angiogenic factors, thus promoting a microenvironmental shift toward a more regenerative milieu. CX3CL1 also increases in human skeletal muscle after exercise. Notably, an interaction between CCL2/CCR2 and CX3CL1/CX3CR1 chemokine systems in modulating $\mathrm{M} \Phi$ function during regeneration exists; the impaired $\mathrm{M} \Phi$ infiltration in $C C L 2^{-/-}$mice is rescued by $C X 3 C R 1$ deficiency through enhanced $\mathrm{M} \Phi$-dependent ApoE production, improving $\mathrm{M} \Phi$ phagocytic activity and compensating for defective monocyte recruitment (7).

2. Cross-talk MФ: FAPs. FAPs are multipotent mesenchymal progenitors of fibroblasts and adipocytes, residing in the skeletal muscle interstitium (Fig. 15) and expressing the surface markers platelet-derived growth factor receptoralpha and Sca1. Muscle regeneration is associated with an early increase of FAPs that provide signals promoting MPCs proliferation and differentiation, as observed in vivo (129, 149, 295, 352). For instance, IL-6 is upregulated in FAPs from day 2 to 5 on muscle damage in mice and enhances MPCs commitment (149). FAPs-derived Follistatin, a TGF$\beta$-superfamily "bio-neutralizer," is crucial for proper myotube formation, also improving muscle repair by increasing MФ and Pax7-positive cell density $(228,388)$. FAPs are also responsible for ECM deposition during muscle regeneration and, once muscle injury is fully repaired, FAPs return to quiescence $(16,352)$.

On acute muscle injury, ECM deposition is a transient and beneficial event supporting regeneration by providing a scaffold for regenerating myofibers. In pathological conditions, for example, muscular dystrophies, connective tissue production is not reversible and leads to myofiber replacement with fibrotic scars and fat deposition (Fig. 13A, Sirius) $(228,284)$. The persistence of activated FAPs (also induced by chronic inflammation) in the damaged muscle is associated with increased collagen and pro-fibrotic factor production, impairing the niche for proper SC activation and differentiation and exacerbating the degenerative phenotype $(59,267)$. Moreover, in injured muscles, FAPs are able to differentiate into intramuscular adipocytes by undefined mechanisms (76). Based on these considerations, the balance between FAPs activation and FAPs apoptosis is crucial for determining the extent of fibrosis, and the modulation of signaling pathways that fine-tune this balance represents a potential therapeutic approach (178).

FAPs are regulated by signals deriving from other resident muscle cells whose behavior is also influenced by FAPs, in a complex network of reciprocal functional interactions (16). Chronic inflammation is a driving force of fibrosis, with several cell types interplaying with inflammatory $\mathrm{M} \Phi$ and contributing to ECM accumulation (194); if M1 were to persist in damaged muscles, this would contribute to fibrosis. As an example, fibrinogen, an ECM factor accumulated in muscles of mdx and DMD patients, binds Mac-1 on the MФ surface, thus upregulating IL- $1 \beta$ and other pro-inflammatory cytokines. IL- $1 \beta$ induces MФ-dependent TGF- $\beta$ expression, which increases muscle fibroblasts collagen production (Fig. 16) (362); compromising fibrinogen-Mac1 interaction in mdx mice decreases inflammation and improves muscle regeneration (361).

Recently, Lemos et al. demonstrated that $М \Phi$-produced soluble factors modulate ECM production and FAPs apoptosis balance in murine models of acute and chronic muscle damage (178). M1-produced TNF $\alpha$ mediates FAPs apoptosis that is necessary to reduce the number of FAPs and to limit fibrosis. M2-produced TGF- $\beta$ counteracts FAPs apoptosis, triggering FAP differentiation in matrix-producing cells (often referred to as $\alpha$-SMA ${ }^{+}$myofibroblasts) providing ECM components such as collagen isoforms, fibronectin, and laminin (16). Accordingly, blocking $M \Phi$ infiltration in regenerative murine muscles, by $C C R 2$ ablation or by $\mathrm{TNF} \alpha$ expression inhibition, decreases FAPs apoptosis and increases fibrotic tissue deposition (178).

Moreover, the tyrosine kinase inhibitors nilotinib and imatinib induce FAPs apoptosis, thereby reducing muscle fibrosis in mdx mice $(136,178)$. However, nilotinib-mediated FAPs expansion inhibition also impairs proper muscle regeneration in a murine model of acute muscle damage, since it negatively affects SC expansion. This underlines the relevance of the FAPs trophic supportive function exerted on SCs (94). Besides $M \Phi$, other inflammatory cells affect FAPs activation during muscle regeneration; in particular, eosinophil-secreted IL-4 induces FAPs proliferation, supporting SC myogenesis and inhibiting FAPs differentiation toward adipocytes (129).

Moreover, additional cell types other than FAPs are involved in collagen deposition during the progression of chronic muscle diseases in mice, for example, pericytes (81), SCs undergoing fibrogenic conversion $(18,273)$, and $\mathrm{M} \Phi$-dependent endothelial-mesenchymal transition of endothelium-derived progenitors (400).

We illustrated how $\mathrm{M} \Phi$ recruitment to damaged muscles is crucial for proper regeneration; in particular, a sequential recruitment of different $M \Phi$ subtypes, with $M 1$ followed by M2, is necessary, since at each stage of regeneration, a reciprocal cross-talk between muscle cells and differently activated $M \Phi$ subsets mediated by soluble factors is required. 
However, the origin of M1 and M2 in the damaged area of the skeletal muscle is still unclear.

\section{Metabolic Reprogramming of $\mathrm{M \Phi}$ as a Potential Therapeutical Approach to Improve Skeletal Muscle Regeneration}

The concept of $M \Phi$ reprogramming to promote antiinflammatory/regenerative M2 polarization that is able to reduce inflammation is emerging as a new therapeutic immunometabolic approach to promote tissue healing and reduce inflammation in chronic inflammatory diseases such as atherosclerosis, rheumatoid arthritis or multiple sclerosis, as well as type 2 diabetes and obesity while improving aging health. Vice versa, a reprogramming from tumor-promoting M2 to anti-tumor M1 has been hypothesized as a therapy against cancer [for references see Ref. (335)].

The study of the contribution of metabolic pathways and the integration of local and systemic metabolism at the cellular level in regulating immune cell development and function is often referred to as immunometabolism; it further includes studying the role of immune cells in metabolic homeostasis. Manipulating immune cell metabolism can beneficially enhance or temper the immune response, including driving $М \Phi$ polarization and function, useful for the potential treatment of several diseases.

Compared with other strategies such as anti-inflammatory, stem cells-based or antioxidant therapies, approaches based on immunometabolism might have the unique advantage to both reduce inflammation and, at the same time, enhance the tissues' regenerative potential, thus being particularly attractive for chronic inflammatory, degenerative, and metabolic diseases [reviewed in Refs. $(133,152)]$. We will discuss how this is particularly evident in skeletal muscle pathologies, including degenerative myopathies, acute injury, and cachexia, as well as in aging and regenerative medicine, where immunometabolism modulation could treat inflammation as well as improve regeneration. In particular, as mentioned earlier, $M \Phi$ effect on muscle resident cells strongly depends on their polarization status, which might be oriented by immunometabolic strategies (268).

\section{A. Aging}

M1-to-M2 polarization is influenced by a metabolic reprogramming that is induced by circulating FFA. In particular, aged individuals' FFA profile (characterized by an overall circulating FFA increase) impairs M2 activation. Also, circulating $\mathrm{TNF} \alpha$ and IL-6 concentrations increase with age, whereas IL-10 and TGF- $\beta 1$ levels decrease, thus negatively affecting skeletal muscle regeneration; therefore, acting on MФ metabolism might be beneficial (259).

In fact, some nutraceuticals can play a prominent role in modulating skeletal muscle physiology (56); among them, resveratrol, green tea, catechins, and $\beta$-hydroxy- $\beta$ methylbutyrate improve SC function and regeneration in muscles of aged mice after disuse, therefore contributing to reducing sarcopenia $(4,15)$. In particular, resveratrol, also considered an "exercise mimetic," promotes murine M2 polarization in vitro; however, whether this mediates the beneficial effect of resveratrol on skeletal muscle regeneration or not needs to be clarified $(53,177,292)$.

\section{B. Duchenne muscular dystrophy}

The balance between different $M \Phi$ subpopulations can determine the progression of dystrophy in mdx mice; drugs that are able to modify this balance might attenuate symptoms of muscular dystrophies. Anti-cytokine drugs favoring a pro-regenerative M2 phenotype might be used, as observed in murine models $(333,363)$. Also, corticosteroids are used with some success to treat DMD, as observed in mice and humans; however, the side effects of these drugs often outweigh their benefits. Interestingly, HDAC inhibitors counteract disease progression in mice by directly acting on muscle cells and also by modulating $\mathrm{M} \Phi$ polarization toward M2 (216). Moreover, the FA EPA increases IL-10 expression and reduces IFN $\gamma$ levels, thus promoting M2 polarization, which decreases inflammation in muscles of dystrophic mice (33). Similarly, the SIRT1-activator resveratrol promotes M2 polarization, decreases inflammation, and increases utrophin expression in mdx mice (112).

\section{Regenerative medicine}

Since regeneration of injured skeletal muscle depends on M1-to-M2 phenotypic transition, in a tissue engineering context, after implantation of the artificial device, the control of M1-to-M2 progression might ensure a proper and timely coordinated transition from the inflammatory to the healing stage. Notably, it has recently been shown that degradation products from the mammalian ECM biologic scaffolds used for skeletal muscle reconstruction in regenerative medicine promote alternative $\mathrm{M} 2$ polarization in in vitro mechanically loaded murine $\mathrm{M} \Phi$, thus facilitating migration and myogenesis of MPCs in vivo and, ultimately, stimulating tissue repair (82, 298, 314).

This IL-4-dependent pro-regenerative response is characterized by cycolooxygenases- 1 and -2 (COX-1/COX-2) activity, which seems to be crucial to allow myogenesis and collagen deposition in the damaged area (69). Resveratrol proM2 activity has been suggested to promote vascularization in tissue engineering applications $(292,349,371)$. Also, transplantation of acellular ECM scaffolds improves performance of diseased muscles by promoting M2 polarization and activating MPCs $(274,278)$; however, other authors showed that, conversely from vital grafts, devitalized grafts were unable to promote an anti-inflammatory phenotype and regeneration in vivo (100).

\section{Obesity-insulin-resistance}

Infiltrating pro-inflammatory $\mathrm{M} \Phi$ characterize the adipose tissue of obese individuals. Pro-inflammatory cytokines impact on the surrounding tissue, which is associated to insulin resistance. Moreover, HFD-mainly saturated FA-also upregulates pro-inflammatory cytokines in skeletal muscle and in skeletal muscle-infiltrating fat, which increase M1 polarization, further contributing to insulin resistance. Therefore, metabolic modulators promoting M2 polarization might be beneficial against insulin resistance, reducing aberrant glucose metabolism; palmitate-treated $\mathrm{M} \Phi$, as well as myoblasts, downregulate $m i R-16$, which is known to impair M1 polarization.

Interestingly, MФ over-expression of miR-16 enhances insulin sensitivity in co-cultured murine myoblasts (330). 
In addition, a major contributor to the insulin-resistance onset might be an altered and pro-oxidant cellular redox state, which decreases NADPH levels that, in turn, activate glucose-6-phosphate dehydrogenase (G6PDH), which is crucial for NADPH maintenance. G6PDH activity is elevated in adipose tissue and its over-expression causes insulin resistance (176). In such a context, the anti-inflammatory and antioxidant effects of M2 polarization might also help in reducing G6PDH expression.

\section{E. Cachexia}

Some diseases, such as cancer and chronic heart failure $(\mathrm{CHF})$, are characterized by weight and skeletal muscle loss that is referred to as cachexia $(219,368)$. In cachexia, an increased systemic inflammatory state is associated with impaired myogenesis. In this context, the $\mathrm{M} \Phi$-reprogramming approach might be useful to promote anti-inflammatory M2 polarization. Notably, anti-inflammatory therapies have failed in counteracting cachexia (368). Therefore, the МФimmunometabolic approach, which does not merely reduce inflammation but also increases M2 activation, might potentially promote muscle regeneration, limiting muscle loss in cachexia. In this regard, we have recently found that the metabolic modulator trimetazidine (TMZ) enhances myogenesis in cancer cachexia in mice (101), along with stimulating M2 reprogramming in vitro (unpublished observations).

The MФ-reprogramming approach against cachexia of cardiac origin is supported by preclinical studies demonstrating that M2 polarization (e.g., by EPA) is beneficial for the myocardium, by reducing inflammation and promoting cardiac healing after myocardial infarction $(52,111,160$, $326,341)$. M2 polarization also counteracts systemic and plaque inflammation in atherosclerotic disorders, thus reducing CHF-associated factors $(119,299)$. As far as cancerinduced cachexia is concerned, however, it must be taken into account that, although being controversial, the most widely proposed immunometabolic therapy against cancer implies achieving M1 polarization to potentiate elimination of cancer cells by TAM (335). Nevertheless, M $\Phi$ balance might have different effects at different stages of tumor progression; therefore, different time points in the reprogramming therapy targeting either cancer or cachexia might also be considered.

\section{F. Stimuli leading to metabolic reprogramming-mediated $M \Phi$ polarization}

Based on the earlier considerations, metabolic reprogramming stimuli modulating the M1/M2 balance might interfere with skeletal muscle regeneration (300). In this paragraph, we will be considering exercise, calorie restriction (CR), and nutrients. Notably, it must be taken into account that the chosen immunometabolic strategy must be selective to $\mathrm{M} \Phi$ without affecting metabolism of other cells. In fact, besides MФ, other resident muscle cells such as SCs undergo metabolic remodeling during regeneration, switching from FAO to glycolysis during the transition from quiescence to proliferation. Moreover, metabolic reprogramming and metabolic substrate utilization shift occur during cell differentiation, when mitochondrial activity increases in most cell types, including SCs.
1. Exercise. Moderate training modulates $M \Phi$ activation by stimulating M1-to-M2 polarization and exerting a global anti-inflammatory effect in multiple organs [reviewed in Refs. $(109,315)]$. For example, in HFD-induced adipose tissue of obese mice, exercise inhibits inflammation by accelerating M1-to-M2 polarization (157, 184, 193, 252). HFDdriven M1 accumulation in white adipose tissue correlates with insulin resistance in obese individuals (380); thanks to its anti-inflammatory effect, exercise improves insulin sensitivity. Also, in rats with nonalcoholic fatty liver disease, moderate exercise increases hepatic M2 polarization (183). Exercise suppresses IL-12 production, a stimulator of IFN $\gamma$, and reduces $\beta 2$-adrenergic receptors in monocytes and $\mathrm{M} \Phi$ by modulating TLR4 signaling (315).

In skeletal muscle, physical activity stimulates the release of myokines, among which cytokines are related to $\mathrm{M} 1 / \mathrm{M} 2$ ratio regulation (e.g., IL-6, TNF $\alpha$, and IL-10) and are involved in skeletal muscle regeneration. Exercise also triggers skeletal muscle $P G C$ - $1 \alpha$ over-expression; PGC- $1 \alpha$ coordinates energy production, modulates myofiber metabolism, and regulates exercise-induced phenotypic adaptation (91). As discussed earlier, $P G C-1 \alpha$ is upregulated in M2. PGC- $1 \alpha$ also stimulates M2 polarization by accelerating necrotic resolution and counteracting fibrosis and muscle wasting in regenerating murine skeletal muscle (72). Muscle regeneration is characterized by a PGC- $1 \alpha$-dependent increased mitochondrial biogenesis and activity. PGC- $1 \alpha$ also contributes to the fast-toslow myofiber conversion after muscle injury in mice, which also influences damaged muscle recovery $(80,204,369)$.

An anti-inflammatory role for PGC- $1 \alpha$ has been reported in cultured muscle cells with PGC- $1 \alpha$ downregulating NF- $\kappa \mathrm{B}$ (72). Interestingly, exercise-induced PGC-1 $\alpha$ upregulation also plays an immunomodulatory role in skeletal muscle by influencing cytokine expression; a PGC- $1 \alpha$-dependent Btype natriuretic peptide (BNP) production in myofibers induces M2 polarization, playing an anti-inflammatory and pro-repair role. Therefore, BNP might be considered a novel PGC-1 $\alpha$-dependent myokine mediating the cross-talk between tissue resident $\mathrm{M} \Phi$ and skeletal muscle, as observed in mice (96). In addition, the myokine irisin-upregulated by exercise-induced PGC- $1 \alpha$-also suppresses inflammation and stimulates M2 polarization in vitro (74).

Gordon et al. showed that resistance exercise enhances M2-associated gene expression in human skeletal muscle (113) and also reduces stress response, improves glucose metabolism, mitochondrial activity, and OxPhos, thus being protective for skeletal muscle (113). МФ activation seems to be relevant in maintaining skeletal muscle energy metabolism, and the transcription factor $\mathrm{C} / \mathrm{EBP} \alpha$ controls both $\mathrm{M} 1-$ and M2 polarization; indeed, in the skeletal muscle of $\mathrm{C} /$ $\mathrm{EBP} \alpha-\mathrm{KO}$ mice, mitochondrial respiration and $\mathrm{FAO}$ are reduced, consistently with an overall decreased exercise capacity of the animal (173).

The effect of exercise strongly depends on its modality, intensity, and timing. Even when over-vigorous exercise results in damage, it still promotes M2 polarization and increased myogenesis in skeletal muscle despite $\mathrm{TNF} \alpha$ increase, as observed in rats (215). Moreover, $\mathrm{pO}_{2}$ decreases in skeletal muscle also on intense exercise, activating HIF, which promotes capillarization and also acts on MФ. M1-toM2 polarization influences myogenesis in vitro in co-cultures of myoblasts with murine $\mathrm{M} \Phi$, whereas few data are 
available regarding the effect of $\mathrm{M} \Phi$ polarization on myogenic cells in vivo as a consequence of physical exercise.

2. Calorie restriction. CR implies a negative energy balance stimulating adaptive metabolic changes with many positive effects such as lifespan extension, delayed ageassociated disease onset, and improvement of metabolic health. Studies of CR effects on MФ polarization in mice mainly concern white adipose tissue, where it has been found that CR leads, by an IL-4R $\alpha$ - and STAT6-dependent signaling, to $\mathrm{M} 2$ polarization and metabolic improvement (88). Mild CR contributes to transient M2 accumulation in inflamed adipose tissue of obese subjects, where M2 supports remodeling of altered adipose tissue and enhances the formation of healthy metabolically flexible adipocytes that are able to control tissue FA levels, triglycerides/FA cycling, and OxPhos (202).

Therefore, M1-to-M2 switching supports healthy adipose tissue via the maintenance of metabolically beneficial МФ. It has also been found that $\mathrm{CR}$ promotes $\mathrm{M} 2$ activation in adipose tissue, in both mice and humans, by increasing the appetitereducing neuropeptide-FF (NPFF) plasma levels. NPFF upregulates $I L-4 R-\alpha, A r g 1, I L-10$, and alkylglycerol monooxygenase; enhances p-STAT6 stability and, thus, M2 polarization (375).

3. Nutrients. MФ-specific PPAR $\gamma$ deletion leads to OxPhos gene downregulation in skeletal muscle and also in the liver, indicating that resident $\mathrm{M} \Phi$ have a beneficial role in regulating nutrient homeostasis (249). Besides resveratrol (see sections V and VII), other nutrients impinging on metabolism, such as amino acids, n-3 polyunsaturated FA (e.g., EPA or DHA), polyphenols, and vitamin D, improve skeletal muscle regeneration by targeting immune and muscle cells $(73,329)$. Indeed, the bioactive form of vitamin D3, 1,25(OH)2D3, inhibits M1 activation and promotes M2 activation, an effect also observed in vivo (390) and being mediated by PPAR $\gamma$ (395). A critical role for the MФ vitamin $\mathrm{D}$ receptor in the inflammatory response to injury has also been reported in mice (316). Moreover, although controversial, a beneficial role for vitamin D in human skeletal muscle regeneration has been proposed $(32,254,266,318)$.

Cocoa polyphenolic extract influences $\mathrm{M} \Phi$ metabolism by promoting oxidative pathways and M2 polarization in human $\mathrm{M} \Phi$ in vitro (79). Pomegranate and its polyphenols promote M1-to-M2 switch in murine MФ (2), and grape seed-derived polyphenols (proanthocyanidolic oligomers) accelerate muscle regeneration in rats by activating SCs and by promoting an anti-inflammatory switch (165). Interestingly, polyamines are able to drive M2 polarization in murine $\mathrm{M} \Phi$ (354) and also to promote repair, fibrosis, and tissue remodeling in mice (272).

Sirtuins play a crucial role in skeletal muscle physiology; indeed, SIRT1 inactivation in skeletal muscle impairs muscle regeneration $(293,348)$. Based on the ability of some sirtuins to modulate metabolism, promoting an M1-to-M2 transition in vivo (see section $\mathrm{V})(17,75,138,153)$, a potential $\mathrm{M} \Phi$ mediated effect of these deacetylases on skeletal muscle regeneration might be worth further investigation. SIRT1 activators are considered "exercise mimetics" and, as reported earlier, other "exercise mimetics" - that is, resveratrol and
AICAR - as well as metformin might drive M2 polarization also in vivo $(21,44,48,239,240)$.

More recently, the metabolic modulator TMZ (144) has also been found to act as an "exercise mimetic"; TMZ increases oxidative metabolism while enhancing skeletal muscle myogenesis $(92,101,219)$. Moreover, TMZ reduces the expression of pro-inflammatory cytokines in LPSstimulated $\mathrm{M} \Phi$ and in a murine model of myocardial dysfunction $(5,45,84)$, stimulating M2 reprogramming in vitro (unpublished observations). This makes this drug and, possibly, other metabolic modulators $(161,345)$ promising candidates for further investigation in the attempt to find new treatments that are able to reprogram $\mathrm{M} \Phi$ metabolism.

\section{Conclusions}

In conclusion, $\mathrm{M} \Phi$ might assume different features and play different roles following specific activation routes, leading to the acquisition of two extreme phenotypes that are referred to as pro-inflammatory M1 and anti-inflammmatory/regenerative M2, which, simplistically, resume a much higher degree of heterogeneity. M1 and M2 subsets are characterized by a differential expression of cytokines, chemokines, and surface markers, by different molecular signatures and transcriptional regulation, and also by different metabolic features specifically illustrated here. Importantly, differently polarized $M \Phi$ play different roles, and $\mathrm{M} \Phi$ are extremely plastic cells that are able to readily switch their phenotype and function if properly stimulated.

In particular, the role of differentially polarized $\mathrm{M} \Phi$ in skeletal muscle regeneration has been discussed here; coordinated МФ-phenotype transition is crucial to allow successful muscle regeneration, and promoting M2 polarization potentiates the tissues' regenerative phase. Specifically, the modulation of $\mathrm{M} \Phi$ metabolism by immunometabolic strategies might be an appealing tool, which is possibly able to drive specific $М \Phi$ polarization routes and to open up a new scenario in terms of rehabilitative protocol design since it represents a potential therapeutic approach for several skeletal muscle conditions that might benefit from reduced inflammation and enhanced regeneration.

In the future, in-depth studies will hopefully disclose $\mathrm{M} \Phi$ heterogeneity and the cellular metabolic pathways associated to specific $\mathrm{M} \Phi$ polarization, thus identifying novel immunometabolic molecular targets and bolstering therapeutic interventions for degenerative and immunometabolic diseases.

\section{Acknowledgments}

The authors are grateful to M. Bennet and V.N. Di Robilant for their valuable editorial work. This work was supported by the Italian Ministry of Health (RF-20102318508) to E.F., by AFM-Telethon (\#16772), by NUTRAMED (PON03PE_00078_2) by Duchenne Parent Project Netherlands (\#DeSanta-DPP-NL), and by the Italian Ministry of Education, Universities and Research (MIUR) (IRMI:CTN01_00177_888744) to F.D.S.

\section{References}

1. Acharyya S, Sharma SM, Cheng AS, Ladner KJ, He W, Kline W, Wang H, Ostrowski MC, Huang TH, and Guttridge DC. TNF inhibits Notch-1 in skeletal muscle cells 
by Ezh2 and DNA methylation mediated repression: implications in Duchenne muscular dystrophy. PLoS One 5: e12479, 2010.

2. Aharoni S, Lati Y, Aviram M, and Fuhrman B. Pomegranate juice polyphenols induce a phenotypic switch in macrophage polarization favoring a M2 anti-inflammatory state. Biofactors 41: 44-51, 2015.

3. Akahori H, Karmali V, Polavarapu R, Lyle AN, Weiss D, Shin E, Husain A, Naqvi N, Van Dam R, Habib A, Choi CU, King AL, Pachura K, Taylor WR, Lefer DJ, and Finn AV. CD163 interacts with TWEAK to regulate tissue regeneration after ischaemic injury. Nat Commun 6: 7792, 2015.

4. Alway SE, Myers MJ, and Mohamed JS. Regulation of satellite cell function in sarcopenia. Front Aging Neurosci 6: 246, 2014.

5. Amber KI, Hadi NR, Muhammad-Baqir BM, Jamil DA, and Al-Aubaidy HA. Trimetazidine attenuates the acute inflammatory response induced by Novolimus eluting bioresorbable coronary scaffold implantation. Int J Cardiol 220: 514-519, 2016.

6. Arnold L, Henry A, Poron F, Baba-Amer Y, van Rooijen $\mathrm{N}$, Plonquet A, Gherardi RK, and Chazaud B. Inflammatory monocytes recruited after skeletal muscle injury switch into antiinflammatory macrophages to support myogenesis. J Exp Med 204: 1057-1069, 2007.

7. Arnold L, Perrin H, de Chanville CB, Saclier M, Hermand P, Poupel L, Guyon E, Licata F, Carpentier W, Vilar J, Mounier R, Chazaud B, Benhabiles N, Boissonnas A, Combadiere B, and Combadiere C. CX3CR1 deficiency promotes muscle repair and regeneration by enhancing macrophage ApoE production. Nat Commun 6: 8972, 2015.

8. Auffray C, Fogg D, Garfa M, Elain G, Join-Lambert O, Kayal S, Sarnacki S, Cumano A, Lauvau G, and Geissmann F. Monitoring of blood vessels and tissues by a population of monocytes with patrolling behavior. Science 317: 666-670, 2007.

9. Bae S, Kim H, Lee N, Won C, Kim HR, Hwang YI, Song YW, Kang JS, and Lee WJ. alpha-Enolase expressed on the surfaces of monocytes and macrophages induces robust synovial inflammation in rheumatoid arthritis. $J$ Immunol 189: 365-372, 2012.

10. Bakker $\mathrm{AD}$ and Jaspers RT. IL-6 and IGF-1 signaling within and between muscle and bone: how important is the mTOR pathway for bone metabolism? Curr Osteoporos Rep 13: 131-139, 2015.

11. Banerjee S, Cui H, Xie N, Tan Z, Yang S, Icyuz M, Thannickal VJ, Abraham E, and Liu G. miR-125a-5p regulates differential activation of macrophages and inflammation. J Biol Chem 288: 35428-35436, 2013.

12. Barton-Davis ER, Shoturma DI, and Sweeney HL. Contribution of satellite cells to IGF-I induced hypertrophy of skeletal muscle. Acta Physiol Scand 167: 301-305, 1999.

13. Bauer M, Goldstein M, Christmann M, Becker H, Heylmann D, and Kaina B. Human monocytes are severely impaired in base and DNA double-strand break repair that renders them vulnerable to oxidative stress. Proc Natl Acad Sci U S A 108: 21105-21110, 2011.

14. Bauernfeind F, Bartok E, Rieger A, Franchi L, Núñez G, and Hornung V. Cutting edge: reactive oxygen species inhibitors block priming, but not activation, of the NLRP3 inflammasome. J Immunol 187: 613-617, 2011.

15. Bennett BT, Mohamed JS, and Alway SE. Effects of resveratrol on the recovery of muscle mass following disuse in the plantaris muscle of aged rats. PLoS One 8: e83518, 2013.

16. Bentzinger CF, Wang YX, Dumont NA, and Rudnicki MA. Cellular dynamics in the muscle satellite cell niche. EMBO Rep 14: 1062-1072, 2013.

17. Bermudez B, Dahl TB, Medina I, Groeneweg M, Holm S, Montserrat-de la Paz S, Rousch M, Otten J, Herias V, Varela LM, Ranheim T, Yndestad A, Ortega-Gomez A, Abia R, Nagy L, Aukrust P, Muriana FJG, Halvorsen B, and Biessen EAL. Leukocyte overexpression of intracellular NAMPT attenuates atherosclerosis by regulating PPARgamma-dependent monocyte differentiation and function. Arterioscler Thromb Vasc Biol 37: 1157-1167, 2017.

18. Biressi S, Miyabara EH, Gopinath SD, Carlig PM, and Rando TA. A Wnt-TGFbeta2 axis induces a fibrogenic program in muscle stem cells from dystrophic mice. Sci Transl Med 6: 267ra176, 2014.

19. Bjornson CR, Cheung TH, Liu L, Tripathi PV, Steeper KM, and Rando TA. Notch signaling is necessary to maintain quiescence in adult muscle stem cells. Stem Cells 30: 232-242, 2012.

20. Bogdan C. Nitric oxide synthase in innate and adaptive immunity: an update. Trends Immunol 36: 161-178, 2015.

21. Börgeson E, Wallenius V, Syed GH, Darshi M, Lantero Rodriguez J, Biörserud C, Ragnmark Ek M, Björklund P, Quiding-Järbrink M, Fändriks L, Godson C, and Sharma K. AICAR ameliorates high-fat diet-associated pathophysiology in mouse and ex vivo models, independent of adiponectin. Diabetologia 60: 729-739, 2017.

22. Bories GFP and Leitinger N. Macrophage metabolism in atherosclerosis. FEBS Lett 591: 3042-3060, 2017.

23. Bosurgi L, Brunelli S, Rigamonti E, Monno A, Manfredi AA, and Rovere-Querini P. Vessel-associated myogenic precursors control macrophage activation and clearance of apoptotic cells. Clin Exp Immunol 179: 62-67, 2015.

24. Bosurgi L, Corna G, Vezzoli M, Touvier T, Cossu G, Manfredi AA, Brunelli S, and Rovere-Querini P. Transplanted mesoangioblasts require macrophage IL-10 for survival in a mouse model of muscle injury. $J$ Immunol 188: 6267-6277, 2012.

25. Brack AS, Conboy IM, Conboy MJ, Shen J, and Rando TA. A temporal switch from notch to Wnt signaling in muscle stem cells is necessary for normal adult myogenesis. Cell Stem Cell 2: 50-59, 2008.

26. Brigitte M, Schilte C, Plonquet A, Baba-Amer Y, Henri A, Charlier C, Tajbakhsh S, Albert M, Gherardi RK, and Chrétien F. Muscle resident macrophages control the immune cell reaction in a mouse model of notexin-induced myoinjury. Arthritis Rheum 62: 268279, 2010.

27. Bronte V and Zanovello P. Regulation of immune responses by L-arginine metabolism. Nat Rev Immunol 5: 641-654, 2005.

28. Brüne B, Dehne N, Grossmann N, Jung M, Namgaladze $\mathrm{D}$, Schmid T, von Knethen A, and Weigert A. Redox control of inflammation in macrophages. Antioxid Redox Signal 19: 595-637, 2013.

29. Burzyn D, Kuswanto W, Kolodin D, Shadrach JL, Cerletti M, Jang Y, Sefik E, Tan TG, Wagers AJ, Benoist C, and Mathis D. A special population of regulatory $\mathrm{T}$ cells potentiates muscle repair. Cell 155: 1282-1295, 2013.

30. Byles V, Covarrubias AJ, Ben-Sahra I, Lamming DW, Sabatini DM, Manning BD, and Horng T. The TSC- 
mTOR pathway regulates macrophage polarization. Nat Commun 4: 2834, 2013.

31. Bylund J, Brown KL, Movitz C, Dahlgren C, and Karlsson A. Intracellular generation of superoxide by the phagocyte NADPH oxidase: how, where, and what for? Free Radic Biol Med 49: 1834-1845, 2010.

32. Camperi A, Pin F, Costamagna D, Penna F, Menduina ML, Aversa Z, Zimmers T, Verzaro R, Fittipaldi R, Caretti G, Baccino FM, Muscaritoli M, and Costelli P. Vitamin D and VDR in cancer cachexia and muscle regeneration. Oncotarget 8: 21778-21793, 2017.

33. Carvalho SC, Apolinário LM, Matheus SM, Santo Neto H, and Marques MJ. EPA protects against muscle damage in the mdx mouse model of Duchenne muscular dystrophy by promoting a shift from the M1 to M2 macrophage phenotype. J Neuroimmunol 264: 41-47, 2013.

34. Castiglioni A, Corna G, Rigamonti E, Basso V, Vezzoli M, Monno A, Almada AE, Mondino A, Wagers AJ, Manfredi AA, and Rovere-Querini P. FOXP3+ T cells recruited to sites of sterile skeletal muscle injury regulate the fate of satellite cells and guide effective tissue regeneration. PLoS One 10: e0128094, 2015.

35. Cervelli M, Fratini E, Amendola R, Bianchi M, Signori E, Ferraro E, Lisi A, Federico R, Marcocci L, and Mariottini P. Increased spermine oxidase (SMO) activity as a novel differentiation marker of myogenic $\mathrm{C} 2 \mathrm{C} 12$ cells. Int $J$ Biochem Cell Biol 41: 934-944, 2009.

36. Chakkalakal JV, Christensen J, Xiang W, Tierney MT, Boscolo FS, Sacco A, and Brack AS. Early forming label-retaining muscle stem cells require p27kip1 for maintenance of the primitive state. Development 141: 1649-1659, 2014.

37. Chakravarthy MV, Abraha TW, Schwartz RJ, Fiorotto ML, and Booth FW. Insulin-like growth factor-I extends in vitro replicative life span of skeletal muscle satellite cells by enhancing G1/S cell cycle progression via the activation of phosphatidylinositol 3'-kinase/Akt signaling pathway. J Biol Chem 275: 35942-35952, 2000.

38. Chang CH, Curtis JD, Maggi LB, Jr., Faubert B, Villarino AV, O'Sullivan D, Huang SC, van der Windt GJ, Blagih J, Qiu J, Weber JD, Pearce EJ, Jones RG, and Pearce EL. Posttranscriptional control of $\mathrm{T}$ cell effector function by aerobic glycolysis. Cell 153: 1239-1251, 2013.

39. Chao LK, Liao PC, Ho CL, Wang EI, Chuang CC, Chiu HW, Hung LB, and Hua KF. Anti-inflammatory bioactivities of honokiol through inhibition of protein kinase $\mathrm{C}$, mitogen-activated protein kinase, and the NF-kappaB pathway to reduce LPS-induced TNFalpha and NO expression. J Agric Food Chem 58: 3472-3478, 2010.

40. Chargé SB and Rudnicki MA. Cellular and molecular regulation of muscle regeneration. Physiol Rev 84: 209 238, 2004.

41. Chazaud B. Macrophages: supportive cells for tissue repair and regeneration. Immunobiology 219: 172-178, 2014.

42. Chazaud B, Brigitte M, Yacoub-Youssef H, Arnold L, Gherardi R, Sonnet C, Lafuste P, and Chretien F. Dual and beneficial roles of macrophages during skeletal muscle regeneration. Exerc Sport Sci Rev 37: 18-22, 2009.

43. Chazaud B, Sonnet C, Lafuste P, Bassez G, Rimaniol AC, Poron F, Authier FJ, Dreyfus PA, and Gherardi RK. Satellite cells attract monocytes and use macrophages as a support to escape apoptosis and enhance muscle growth. $J$ Cell Biol 163: 1133-1143, 2003.
44. Chen H, Ji H, Zhang M, Liu Z, Lao L, Deng C, Chen J, and Zhong G. An agonist of the protective factor SIRT1 improves functional recovery and promotes neuronal survival by attenuating inflammation after spinal cord injury. J Neurosci 37: 2916-2930, 2017.

45. Chen J, Lai J, Yang L, Ruan G, Chaugai S, Ning Q, Chen $\mathrm{C}$, and Wang DW. Trimetazidine prevents macrophagemediated septic myocardial dysfunction via activation of the histone deacetylase sirtuin 1. Br J Pharmacol 173: 545-561, 2016.

46. Chen M, Zhang J, Hu F, Liu S, and Zhou Z. Metformin affects the features of a human hepatocellular cell line (HepG2) by regulating macrophage polarization in a coculture microenviroment. Diabetes Metab Res Rev 31: 781-789, 2015.

47. Chen P, Cescon M, Zuccolotto G, Nobbio L, Colombelli C, Filaferro M, Vitale G, Feltri ML, and Bonaldo P. Collagen VI regulates peripheral nerve regeneration by modulating macrophage recruitment and polarization. Acta Neuropathol 129: 97-113, 2015.

48. Chen YX, Zhang M, Cai Y, Zhao Q, and Dai W. The Sirt1 activator SRT1720 attenuates angiotensin II-induced atherosclerosis in $\mathrm{apoE}^{-} /$mice through inhibiting vascular inflammatory response. Biochem Biophys Res Commun 465: 732-738, 2015.

49. Cheng M, Nguyen MH, Fantuzzi G, and Koh TJ. Endogenous interferon-gamma is required for efficient skeletal muscle regeneration. Am J Physiol Cell Physiol 294: C1183C1191, 2008.

50. Chiang CF, Chao TT, Su YF, Hsu CC, Chien CY, Chiu KC, Shiah SG, Lee CH, Liu SY, and Shieh YS. Metformintreated cancer cells modulate macrophage polarization through AMPK-NF-kB signaling. Oncotarget 8: 2070620718, 2017.

51. Choi SH, Aid S, Kim HW, Jackson SH, and Bosetti F. Inhibition of NADPH oxidase promotes alternative and anti-inflammatory microglial activation during neuroinflammation. J Neurochem 120: 292-301, 2012.

52. Choo EH, Lee JH, Park EH, Park HE, Jung NC, Kim TH, Koh YS, Kim E, Seung KB, Park C, Hong KS, Kang K, Song JY, Seo HG, Lim DS, and Chang K. Infarcted myocardium-primed dendritic cells improve remodeling and cardiac function after myocardial infarction by modulating the regulatory $\mathrm{T}$ cell and macrophage polarization. Circulation 135: 1444-1457, 2017.

53. Chung EY, Kim BH, Hong JT, Lee CK, Ahn B, Nam SY, Han SB, and Kim Y. Resveratrol down-regulates interferongamma-inducible inflammatory genes in macrophages: molecular mechanism via decreased STAT-1 activation. $J$ Nutr Biochem 22: 902-909, 2011.

54. Ciciliot $S$ and Schiaffino S. Regeneration of mammalian skeletal muscle. Basic mechanisms and clinical implications. Curr Pharm Des 16: 906-914, 2010.

55. Cimen H, Han MJ, Yang Y, Tong Q, Koc H, and Koc EC. Regulation of succinate dehydrogenase activity by SIRT3 in mammalian mitochondria. Biochemistry 49: 304-311, 2010.

56. Close GL, Hamilton DL, Philp A, Burke LM, and Morton JP. New strategies in sport nutrition to increase exercise performance. Free Radic Biol Med 98: 144-158, 2016.

57. Colegio OR, Chu NQ, Szabo AL, Chu T, Rhebergen AM, Jairam V, Cyrus N, Brokowski CE, Eisenbarth SC, Phillips GM, Cline GW, Phillips AJ, and Medzhitov R. Functional 
polarization of tumour-associated macrophages by tumourderived lactic acid. Nature 513: 559-563, 2014.

58. Conboy IM and Rando TA. The regulation of Notch signaling controls satellite cell activation and cell fate determination in postnatal myogenesis. Dev Cell 3: 397409, 2002.

59. Contreras O, Rebolledo DL, Oyarzún JE, Olguín HC, and Brandan E. Connective tissue cells expressing fibro/adipogenic progenitor markers increase under chronic damage: relevance in fibroblast-myofibroblast differentiation and skeletal muscle fibrosis. Cell Tissue Res 364: 647-660, 2016.

60. Contreras-Shannon V, Ochoa O, Reyes-Reyna SM, Sun D, Michalek JE, Kuziel WA, McManus LM, and Shireman PK. Fat accumulation with altered inflammation and regeneration in skeletal muscle of CCR2-/- mice following ischemic injury. Am J Physiol Cell Physiol 292: C953C967, 2007.

61. Cordes T, Wallace M, Michelucci A, Divakaruni AS, Sapcariu SC, Sousa C, Koseki H, Cabrales P, Murphy AN, Hiller K, and Metallo CM. Immunoresponsive gene 1 and itaconate inhibit succinate dehydrogenase to modulate intracellular succinate levels. J Biol Chem 291: 1427414284, 2016.

62. Corraliza IM, Soler G, Eichmann K, and Modolell M. Arginase induction by suppressors of nitric oxide synthesis (IL-4, IL-10 and PGE2) in murine bone-marrowderived macrophages. Biochem Biophys Res Commun 206: 667-673, 1995.

63. Covarrubias AJ, Aksoylar HI, and Horng T. Control of macrophage metabolism and activation by mTOR and Akt signaling. Semin Immunol 27: 286-296, 2015.

64. Cramer T, Yamanishi Y, Clausen BE, Förster I, Pawlinski R, Mackman N, Haase VH, Jaenisch R, Corr M, Nizet V, Firestein GS, Gerber HP, Ferrara N, and Johnson RS. HIF1alpha is essential for myeloid cell-mediated inflammation. Cell 112: 645-657, 2003.

65. Cruz CM, Rinna A, Forman HJ, Ventura AL, Persechini PM, and Ojcius DM. ATP activates a reactive oxygen species-dependent oxidative stress response and secretion of proinflammatory cytokines in macrophages. $J$ Biol Chem 282: 2871-2879, 2007.

66. Darnell JE, Jr., Kerr IM, and Stark GR. Jak-STAT pathways and transcriptional activation in response to IFNs and other extracellular signaling proteins. Science 264: 1415-1421, 1994.

67. Davies LC, Jenkins SJ, Allen JE, and Taylor PR. Tissueresident macrophages. Nat Immunol 14: 986-995, 2013.

68. De Santa F, Narang V, Yap ZH, Tusi BK, Burgold T, Austenaa L, Bucci G, Caganova M, Notarbartolo S, Casola S, Testa G, Sung WK, Wei CL, and Natoli G. Jmjd3 contributes to the control of gene expression in LPSactivated macrophages. EMBO J 28: 3341-3352, 2009.

69. Dearth CL, Slivka PF, Stewart SA, Keane TJ, Tay JK, Londono R, Goh Q, Pizza FX, and Badylak SF. Inhibition of COX1/2 alters the host response and reduces ECM scaffold mediated constructive tissue remodeling in a rodent model of skeletal muscle injury. Acta Biomater 31: 50-60, 2016.

70. Deng B, Wehling-Henricks M, Villalta SA, Wang Y, and Tidball JG. IL-10 triggers changes in macrophage phenotype that promote muscle growth and regeneration. $J$ Immunol 189: 3669-3680, 2012.

71. Ding L, Liang G, Yao Z, Zhang J, Liu R, Chen H, Zhou Y, $\mathrm{Wu} \mathrm{H}$, Yang B, and He Q. Metformin prevents cancer metastasis by inhibiting M2-like polarization of tumor associated macrophages. Oncotarget 6: 36441-36455, 2015.

72. Dinulovic I, Furrer R, Di Fulvio S, Ferry A, Beer M, and Handschin C. PGC-1alpha modulates necrosis, inflammatory response, and fibrotic tissue formation in injured skeletal muscle. Skelet Muscle 6: 38, 2016.

73. Domingues-Faria C, Vasson MP, Goncalves-Mendes N, Boirie Y, and Walrand S. Skeletal muscle regeneration and impact of aging and nutrition. Ageing Res Rev 26: 22 36, 2016.

74. Dong J, Dong Y, Dong Y, Chen F, Mitch WE, and Zhang L. Inhibition of myostatin in mice improves insulin sensitivity via irisin-mediated cross talk between muscle and adipose tissues. Int J Obes (Lond) 40: 434-442, 2016.

75. Dong J, Zhang X, Zhang L, Bian HX, Xu N, Bao B, and Liu J. Quercetin reduces obesity-associated ATM infiltration and inflammation in mice: a mechanism including AMPKalpha1/SIRT1. J Lipid Res 55: 363-374, 2014.

76. Dong Y, Silva KA, Dong Y, and Zhang L. Glucocorticoids increase adipocytes in muscle by affecting IL-4 regulated FAP activity. FASEB J 28: 4123-4132, 2014.

77. Du H, Shih CH, Wosczyna MN, Mueller AA, Cho J, Aggarwal A, Rando TA, and Feldman BJ. Macrophagereleased ADAMTS1 promotes muscle stem cell activation. Nat Commun 8: 669, 2017.

78. Duffield JS, Lupher M, Thannickal VJ, and Wynn TA. Host responses in tissue repair and fibrosis. Annu Rev Pathol 8: 241-276, 2013.

79. Dugo L, Belluomo MG, Fanali C, Russo M, Cacciola F, Maccarrone M, and Sardanelli AM. Effect of cocoa polyphenolic extract on macrophage polarization from proinflammatory M1 to anti-inflammatory M2 state. Oxid Med Cell Longev 2017: 6293740, 2017.

80. Duguez S, Féasson L, Denis C, and Freyssenet D. Mitochondrial biogenesis during skeletal muscle regeneration. Am J Physiol Endocrinol Metab 282: E802-E809, 2002.

81. Dulauroy S, Di Carlo SE, Langa F, Eberl G, and Peduto L. Lineage tracing and genetic ablation of ADAM12(+) perivascular cells identify a major source of profibrotic cells during acute tissue injury. Nat Med 18: 1262-1270, 2012.

82. Dziki JL, Giglio RM, Sicari BM, Wang DS, Gandhi RM, Londono R, Dearth CL, and Badylak SF. The effect of mechanical loading upon extracellular matrix bioscaffoldmediated skeletal muscle remodeling. Tissue Eng Part A 24: 34-46, 2018.

83. El Kasmi KC, Qualls JE, Pesce JT, Smith AM, Thompson RW, Henao-Tamayo M, Basaraba RJ, König T, Schleicher U, Koo MS, Kaplan G, Fitzgerald KA, Tuomanen EI, Orme IM, Kanneganti TD, Bogdan C, Wynn TA, and Murray PJ. Toll-like receptor-induced arginase 1 in macrophages thwarts effective immunity against intracellular pathogens. Nat Immunol 9: 1399-1406, 2008.

84. El-Sherbeeny NA and Attia GM. The protective effect of trimetazidine against cisplatin-induced nephrotoxicity in rats. Can J Physiol Pharmacol 94: 745-751, 2016.

85. Esteban-Martinez L and Boya P. BNIP3L/NIX-dependent mitophagy regulates cell differentiation via metabolic reprogramming. Autophagy 14: 915-917, 2018.

86. Everts B, Amiel E, Huang SC, Smith AM, Chang $\mathrm{CH}$, Lam WY, Redmann V, Freitas TC, Blagih J, van der Windt GJ, Artyomov MN, Jones RG, Pearce EL, and Pearce EJ. TLR-driven early glycolytic reprogramming 
via the kinases TBK1-IKK $\varepsilon$ supports the anabolic demands of dendritic cell activation. Nat Immunol 15: $323-$ 332, 2014.

87. Everts B, Amiel E, van der Windt GJ, Freitas TC, Chott R, Yarasheski KE, Pearce EL, and Pearce EJ. Commitment to glycolysis sustains survival of NO-producing inflammatory dendritic cells. Blood 120: 1422-1431, 2012.

88. Fabbiano S, Suárez-Zamorano N, Rigo D, VeyratDurebex C, Stevanovic Dokic A, Colin DJ, and Trajkovski M. Caloric restriction leads to browning of white adipose tissue through type 2 immune signaling. Cell Metab 24: 434-446, 2016.

89. Feinberg MW, Cao Z, Wara AK, Lebedeva MA, Senbanerjee $\mathrm{S}$, and Jain MK. Kruppel-like factor 4 is a mediator of proinflammatory signaling in macrophages. $J$ Biol Chem 280: 38247-38258, 2005.

90. Fernando MR, Reyes JL, Iannuzzi J, Leung G, and McKay DM. The pro-inflammatory cytokine, interleukin-6, enhances the polarization of alternatively activated macrophages. PLoS One 9: e94188, 2014.

91. Ferraro E, Giammarioli AM, Chiandotto S, Spoletini I, and Rosano G. Exercise-induced skeletal muscle remodeling and metabolic adaptation: redox signaling and role of autophagy. Antioxid Redox Signal 21: 154-176, 2014.

92. Ferraro E, Pin F, Gorini S, Pontecorvo L, Ferri A, Mollace V, Costelli P, and Rosano G. Improvement of skeletal muscle performance in ageing by the metabolic modulator Trimetazidine. J Cachexia Sarcopenia Muscle 7: 449457, 2016.

93. Fielding RA, Manfredi TJ, Ding W, Fiatarone MA, Evans WJ, and Cannon JG. Acute phase response in exercise. III. Neutrophil and IL-1 beta accumulation in skeletal muscle. Am J Physiol 265: R166-R172, 1993.

94. Fiore D, Judson RN, Low M, Lee S, Zhang E, Hopkins C, Xu P, Lenzi A, Rossi FM, and Lemos DR. Pharmacological blockage of fibro/adipogenic progenitor expansion and suppression of regenerative fibrogenesis is associated with impaired skeletal muscle regeneration. Stem Cell Res 17: 161-169, 2016.

95. Fogg DK, Sibon C, Miled C, Jung S, Aucouturier P, Littman DR, Cumano A, and Geissmann F. A clonogenic bone marrow progenitor specific for macrophages and dendritic cells. Science 311: 83-87, 2006.

96. Furrer R, Eisele PS, Schmidt A, Beer M, and Handschin C. Paracrine cross-talk between skeletal muscle and macrophages in exercise by PGC-1alpha-controlled BNP. Sci Rep 7: 40789, 2017.

97. Galván-Peña S and O'Neill LA. Metabolic reprograming in macrophage polarization. Front Immunol 5: 420, 2014.

98. Gao YT, Panda SP, Roman LJ, Martásek P, Ishimura Y, and Masters BS. Oxygen metabolism by neuronal nitricoxide synthase. J Biol Chem 282: 7921-7929, 2007.

99. Gao Z, Li Y, Wang F, Huang T, Fan K, Zhang Y, Zhong J, Cao Q, Chao T, Jia J, Yang S, Zhang L, Xiao Y, Zhou JY, Feng XH, and Jin J. Mitochondrial dynamics controls antitumour innate immunity by regulating CHIP-IRF1 axis stability. Nat Commun 8: 1805, 2017.

100. Garg K, Ward CL, Rathbone CR, and Corona BT. Transplantation of devitalized muscle scaffolds is insufficient for appreciable de novo muscle fiber regeneration after volumetric muscle loss injury. Cell Tissue Res 358: 857-873, 2014.

101. Gatta L, Vitiello L, Gorini S, Chiandotto S, Costelli P, Giammarioli AM, Malorni W, Rosano G, and Ferraro E.
Modulating the metabolism by trimetazidine enhances myoblast differentiation and promotes myogenesis in cachectic tumor-bearing c26 mice. Oncotarget 8: 113938113956, 2017.

102. Geissmann F, Jung S, and Littman DR. Blood monocytes consist of two principal subsets with distinct migratory properties. Immunity 19: 71-82, 2003.

103. Geissmann F, Manz MG, Jung S, Sieweke MH, Merad M, and Ley K. Development of monocytes, macrophages, and dendritic cells. Science 327: 656-661, 2010.

104. Ghafourifar P and Cadenas E. Mitochondrial nitric oxide synthase. Trends Pharmacol Sci 26: 190-195, 2005.

105. Ginhoux F, Greter M, Leboeuf M, Nandi S, See P, Gokhan S, Mehler MF, Conway SJ, Ng LG, Stanley ER, Samokhvalov IM, and Merad M. Fate mapping analysis reveals that adult microglia derive from primitive macrophages. Science 330: 841-845, 2010.

106. Gladwin MT, Grubina R, and Doyle MP. The new chemical biology of nitrite reactions with hemoglobin: $\mathrm{R}$-state catalysis, oxidative denitrosylation, and nitrite reductase/anhydrase. Acc Chem Res 42: 157-167, 2009.

107. Gleissner CA, Shaked I, Little KM, and Ley K. CXC chemokine ligand 4 induces a unique transcriptome in monocytederived macrophages. J Immunol 184: 4810-4818, 2010.

108. Godwin JW, Pinto AR, and Rosenthal NA. Macrophages are required for adult salamander limb regeneration. Proc Natl Acad Sci U S A 110: 9415-9420, 2013.

109. Goh J, Goh KP, and Abbasi A. Exercise and adipose tissue macrophages: new Frontiers in obesity research? Front Endocrinol (Lausanne) 7: 65, 2016.

110. Gold R, Kappos L, Arnold DL, Bar-Or A, Giovannoni G, Selmaj K, Tornatore C, Sweetser MT, Yang M, Sheikh SI, Dawson KT; DEFINE Study Investigators. Placebocontrolled phase 3 study of oral BG-12 for relapsing multiple sclerosis. N Engl J Med 367: 1098-1107, 2012.

111. Gombozhapova A, Rogovskaya Y, Shurupov V, Rebenkova M, Kzhyshkowska J, Popov SV, Karpov RS, and Ryabov V. Macrophage activation and polarization in post-infarction cardiac remodeling. J Biomed Sci 24: 13, 2017.

112. Gordon BS, Delgado Díaz DC, and Kostek MC. Resveratrol decreases inflammation and increases utrophin gene expression in the mdx mouse model of Duchenne muscular dystrophy. Clin Nutr 32: 104-111, 2013.

113. Gordon PM, Liu D, Sartor MA, IglayReger HB, Pistilli EE, Gutmann L, Nader GA, and Hoffman EP. Resistance exercise training influences skeletal muscle immune activation: a microarray analysis. J Appl Physiol (1985) 112: 443-453, 2012.

114. Gordon S and Martinez FO. Alternative activation of macrophages: mechanism and functions. Immunity 32: 593-604, 2010.

115. Graham RR, Kozyrev SV, Baechler EC, Reddy MV, Plenge RM, Bauer JW, Ortmann WA, Koeuth T, González Escribano MF, Argentine and Spanish Collaborative Groups, Pons-Estel B, Petri M, Daly M, Gregersen PK, Martín J, Altshuler D, Behrens TW, and AlarcónRiquelme ME. A common haplotype of interferon regulatory factor 5 (IRF5) regulates splicing and expression and is associated with increased risk of systemic lupus erythematosus. Nat Genet 38: 550-555, 2006.

116. Gregory CD and Pound JD. Cell death in the neighbourhood: direct microenvironmental effects of apoptosis in normal and neoplastic tissues. J Pathol 223: 177-194, 2011. 
117. Hah YS, Cheon YH, Lim HS, Cho HY, Park BH, Ka SO, Lee YR, Jeong DW, Kim HO, Han MK, and Lee SI. Myeloid deletion of SIRT1 aggravates serum transfer arthritis in mice via nuclear factor-kappaB activation. PLoS One 9: e87733, 2014.

118. Hall CJ, Boyle RH, Astin JW, Flores MV, Oehlers SH, Sanderson LE, Ellett F, Lieschke GJ, Crosier KE, and Crosier PS. Immunoresponsive gene 1 augments bactericidal activity of macrophage-lineage cells by regulating beta-oxidation-dependent mitochondrial ROS production. Cell Metab 18: 265-278, 2013.

119. Halvorsen B, Espeland MZ, Andersen G $\varnothing$, Yndestad A, Sagen EL, Rashidi A, Knudsen EC, Skjelland M, Skagen KR, Krohg-Sørensen K, Holm S, Ritschel V, Holven KB, Biessen EA, Aukrust P, and Dahl TB. Increased expression of NAMPT in PBMC from patients with acute coronary syndrome and in inflammatory M1 macrophages. Atherosclerosis 243: 204-210, 2015.

120. Hamm A, Veschini L, Takeda Y, Costa S, Delamarre E, Squadrito ML, Henze AT, Wenes M, Serneels J, Pucci F, Roncal C, Anisimov A, Alitalo K, De Palma M, and Mazzone M. PHD2 regulates arteriogenic macrophages through TIE2 signalling. EMBO Mol Med 5: 843-857, 2013.

121. Hanna RN, Shaked I, Hubbeling HG, Punt JA, Wu R, Herrley E, Zaugg C, Pei H, Geissmann F, Ley K, and Hedrick CC. NR4A1 (Nur77) deletion polarizes macrophages toward an inflammatory phenotype and increases atherosclerosis. Circ Res 110: 416-427, 2012.

122. Hara M, Yuasa S, Shimoji K, Onizuka T, Hayashiji N, Ohno Y, Arai T, Hattori F, Kaneda R, Kimura K, Makino S, Sano M, and Fukuda K. G-CSF influences mouse skeletal muscle development and regeneration by stimulating myoblast proliferation. J Exp Med 208: 715-727, 2011.

123. Harada T, Tanikawa T, Iwasaki Y, Yamada M, Imai Y, and Miyake M. Phagocytic entry of Legionella pneumophila into macrophages through phosphatidylinositol 3,4,5-trisphosphate-independent pathway. Biol Pharm Bull 35: 1460-1468, 2012.

124. Hard GC. Some biochemical aspects of the immune macrophage. Br J Exp Pathol 51: 97-105, 1970.

125. Hardy D, Besnard A, Latil M, Jouvion G, Briand D, Thépenier C, Pascal Q, Guguin A, Gayraud-Morel B, Cavaillon JM, Tajbakhsh S, Rocheteau P, and Chrétien F. Comparative study of injury models for studying muscle regeneration in mice. PLoS One 11: e0147198, 2016.

126. Haschemi A, Kosma P, Gille L, Evans CR, Burant CF, Starkl P, Knapp B, Haas R, Schmid JA, Jandl C, Amir S, Lubec G, Park J, Esterbauer H, Bilban M, Brizuela L, Pospisilik JA, Otterbein LE, and Wagner O. The sedoheptulose kinase CARKL directs macrophage polarization through control of glucose metabolism. Cell Metab 15: 813-826, 2012.

127. Hasegawa-Moriyama M, Ohnou T, Godai K, Kurimoto T, Nakama M, and Kanmura Y. Peroxisome proliferatoractivated receptor-gamma agonist rosiglitazone attenuates postincisional pain by regulating macrophage polarization. Biochem Biophys Res Commun 426: 76-82, 2012.

128. He C, Ryan AJ, Murthy S, and Carter AB. Accelerated development of pulmonary fibrosis via $\mathrm{Cu}, \mathrm{Zn}$-superoxide dismutase-induced alternative activation of macrophages. J Biol Chem 288: 20745-20757, 2013.

129. Heredia JE, Mukundan L, Chen FM, Mueller AA, Deo RC, Locksley RM, Rando TA, and Chawla A. Type 2 innate signals stimulate fibro/adipogenic progenitors to facilitate muscle regeneration. Cell 153: 376-388, 2013.

130. Hibbs JB, Jr., Taintor RR, and Vavrin Z. Macrophage cytotoxicity: role for L-arginine deiminase and imino nitrogen oxidation to nitrite. Science 235: 473-476, 1987.

131. Hill BG, Dranka BP, Bailey SM, Lancaster JR, Jr., and Darley-Usmar VM. What part of NO don't you understand? Some answers to the cardinal questions in nitric oxide biology. J Biol Chem 285: 19699-19704, 2010.

132. Horsley V, Jansen KM, Mills ST, and Pavlath GK. IL-4 acts as a myoblast recruitment factor during mammalian muscle growth. Cell 113: 483-494, 2003.

133. Hotamisligil GS. Foundations of immunometabolism and implications for metabolic health and disease. Immunity 47: 406-420, 2017.

134. Huang SC, Everts B, Ivanova Y, O’Sullivan D, Nascimento M, Smith AM, Beatty W, Love-Gregory L, Lam WY, O'Neill CM, Yan C, Du H, Abumrad NA, Urban JF, Jr., Artyomov MN, Pearce EL, and Pearce EJ. Cell-intrinsic lysosomal lipolysis is essential for alternative activation of macrophages. Nat Immunol 15: 846-855, 2014.

135. Huang SC, Smith AM, Everts B, Colonna M, Pearce EL, Schilling JD, and Pearce EJ. Metabolic reprogramming mediated by the mTORC2-IRF4 signaling axis is essential for macrophage alternative activation. Immunity 45: 817830, 2016.

136. Huang X, Patel S, Ahmed N, Seiter K, and Liu D. Severe toxicity of skin rash, fever and diarrhea associated with imatinib: case report and review of skin toxicities associated with tyrosine kinase inhibitors. Drug Des Devel Ther 2: 215-219, 2009.

137. Huang Z, Hoffmann FW, Fay JD, Hashimoto AC, Chapagain ML, Kaufusi PH, and Hoffmann PR. Stimulation of unprimed macrophages with immune complexes triggers a low output of nitric oxide by calcium-dependent neuronal nitric-oxide synthase. J Biol Chem 287: 44924502, 2012.

138. Hui X, Zhang M, Gu P, Li K, Gao Y, Wu D, Wang Y, and $\mathrm{Xu}$ A. Adipocyte SIRT1 controls systemic insulin sensitivity by modulating macrophages in adipose tissue. EMBO Rep 18: 645-657, 2017.

139. Incio J, Suboj P, Chin SM, Vardam-Kaur T, Liu H, Hato T, Babykutty S, Chen I, Deshpande V, Jain RK, and Fukumura D. Metformin reduces desmoplasia in pancreatic cancer by reprogramming stellate cells and tumorassociated macrophages. PLoS One 10: e0141392, 2015.

140. Infantino V, Convertini P, Cucci L, Panaro MA, Di Noia MA, Calvello R, Palmieri F, and Iacobazzi V. The mitochondrial citrate carrier: a new player in inflammation. Biochem J 438: 433-436, 2011.

141. Infantino V, Iacobazzi V, Palmieri F, and Menga A. ATPcitrate lyase is essential for macrophage inflammatory response. Biochem Biophys Res Commun 440: 105-111, 2013.

142. Italiani P, and Boraschi D. From monocytes to M1/M2 macrophages: phenotypical vs. functional differentiation. Front Immunol 5: 514, 2014.

143. Izquierdo E, Cuevas VD, Fernández-Arroyo S, RieraBorrull M, Orta-Zavalza E, Joven J, Rial E, Corbi AL, and Escribese MM. Reshaping of human macrophage polarization through modulation of glucose catabolic pathways. J Immunol 195: 2442-2451, 2015.

144. Jaswal JS, Keung W, Wang W, Ussher JR, and Lopaschuk GD. Targeting fatty acid and carbohydrate oxidation-a 
novel therapeutic intervention in the ischemic and failing heart. Biochim Biophys Acta 1813: 1333-1350, 2011.

145. Jha AK, Huang SC, Sergushichev A, Lampropoulou V, Ivanova Y, Loginicheva E, Chmielewski K, Stewart KM, Ashall J, Everts B, Pearce EJ, Driggers EM, and Artyomov MN. Network integration of parallel metabolic and transcriptional data reveals metabolic modules that regulate macrophage polarization. Immunity 42: 419-430, 2015.

146. Jia Y, Han S, Li J, Wang H, Liu J, Li N, Yang X, Shi J, Han J, Li Y, Bai X, Su L, and Hu D. IRF8 is the target of SIRT1 for the inflammation response in macrophages. Innate Immun 23: 188-195, 2017.

147. Jiang H, Shi H, Sun M, Wang Y, Meng Q, Guo P, Cao Y, Chen J, Gao X, Li E, and Liu J. PFKFB3-driven macrophage glycolytic metabolism is a crucial component of innate antiviral defense. J Immunol 197: 2880-2890, 2016.

148. Jiang L, Li X, Zhang Y, Zhang M, Tang Z, and Lv K. Microarray and bioinformatics analyses of gene expression profiles in BALB/c murine macrophage polarization. Mol Med Rep 16: 7382-7390, 2017.

149. Joe AW, Yi L, Natarajan A, Le Grand F, So L, Wang J, Rudnicki MA, and Rossi FM. Muscle injury activates resident fibro/adipogenic progenitors that facilitate myogenesis. Nat Cell Biol 12: 153-163, 2010.

150. Johann AM, von Knethen A, Lindemann D, and Brüne B. Recognition of apoptotic cells by macrophages activates the peroxisome proliferator-activated receptor-gamma and attenuates the oxidative burst. Cell Death Differ 13: 15331540, 2006.

151. Johnson AR, Qin Y, Cozzo AJ, Freemerman AJ, Huang MJ, Zhao L, Sampey BP, Milner JJ, Beck MA, Damania B, Rashid N, Galanko JA, Lee DP, Edin ML, Zeldin DC, Fueger PT, Dietz B, Stahl A, Wu Y, Mohlke KL, and Makowski L. Metabolic reprogramming through fatty acid transport protein 1 (FATP1) regulates macrophage inflammatory potential and adipose inflammation. Mol Metab 5: 506-526, 2016.

152. Juban G and Chazaud B. Metabolic regulation of macrophages during tissue repair: insights from skeletal muscle regeneration. FEBS Lett 591: 3007-3021, 2017.

153. Ka SO, Song MY, Bae EJ, and Park BH. Myeloid SIRT1 regulates macrophage infiltration and insulin sensitivity in mice fed a high-fat diet. J Endocrinol 224: 109-118, 2015.

154. Kadl A, Meher AK, Sharma PR, Lee MY, Doran AC, Johnstone SR, Elliott MR, Gruber F, Han J, Chen W, Kensler T, Ravichandran KS, Isakson BE, Wamhoff BR, and Leitinger $\mathrm{N}$. Identification of a novel macrophage phenotype that develops in response to atherogenic phospholipids via Nrf2. Circ Res 107: 737-746, 2010.

155. Kapoor N, Niu J, Saad Y, Kumar S, Sirakova T, Becerra $\mathrm{E}$, Li X, and Kolattukudy PE. Transcription factors STAT6 and KLF4 implement macrophage polarization via the dual catalytic powers of MCPIP. J Immunol 194: 6011-6023, 2015.

156. Kaufmann SH. Immunology's foundation: the 100-year anniversary of the Nobel Prize to Paul Ehrlich and Elie Metchnikoff. Nat Immunol 9: 705-712, 2008.

157. Kawanishi N, Yano H, Yokogawa Y, and Suzuki K. Exercise training inhibits inflammation in adipose tissue via both suppression of macrophage infiltration and acceleration of phenotypic switching from M1 to M2 macrophages in high-fat-diet-induced obese mice. Exerc Immunol Rev 16: 105-118, 2010.
158. Kharraz Y, Guerra J, Mann CJ, Serrano AL, and MuñozCánoves P. Macrophage plasticity and the role of inflammation in skeletal muscle repair. Mediators Inflamm 2013: 491497, 2013.

159. Kim GD, Das R, Goduni L, McClellan S, Hazlett LD, and Mahabeleshwar GH. Kruppel-like factor 6 promotes macrophage-mediated inflammation by suppressing B cell leukemia/lymphoma 6 expression. J Biol Chem 291: 21271-21282, 2016.

160. Kim YS, Jeong HY, Kim AR, Kim WH, Cho H, Um J, Seo Y, Kang WS, Jin SW, Kim MC, Kim YC, Jung DW, Williams DR, and Ahn Y. Natural product derivative BIO promotes recovery after myocardial infarction via unique modulation of the cardiac microenvironment. Sci Rep 6: 30726, 2016.

161. Komajda M, Tavazzi L, Francq BG, Böhm M, Borer JS, Ford I, Swedberg K; SHIFT Investigators. Efficacy and safety of ivabradine in patients with chronic systolic heart failure and diabetes: an analysis from the SHIFT trial. Eur J Heart Fail 17: 1294-1301, 2015.

162. Kraft-Terry SD and Gendelman HE. Proteomic biosignatures for monocyte-macrophage differentiation. Cell Immunol 271: 239-255, 2011.

163. Kratz M, Coats BR, Hisert KB, Hagman D, Mutskov V, Peris E, Schoenfelt KQ, Kuzma JN, Larson I, Billing PS, Landerholm RW, Crouthamel M, Gozal D, Hwang S, Singh PK, and Becker L. Metabolic dysfunction drives a mechanistically distinct proinflammatory phenotype in adipose tissue macrophages. Cell Metab 20: 614-625, 2014.

164. Krawczyk CM, Holowka T, Sun J, Blagih J, Amiel E, DeBerardinis RJ, Cross JR, Jung E, Thompson CB, Jones $\mathrm{RG}$, and Pearce EJ. Toll-like receptor-induced changes in glycolytic metabolism regulate dendritic cell activation. Blood 115: 4742-4749, 2010.

165. Kruger MJ and Smith C. Postcontusion polyphenol treatment alters inflammation and muscle regeneration. Med Sci Sports Exerc 44: 872-880, 2012.

166. Kuchler L, Giegerich AK, Sha LK, Knape T, Wong MS, Schröder K, Brandes RP, Heide H, Wittig I, Brüne B, and von Knethen A. SYNCRIP-dependent Nox2 mRNA destabilization impairs ROS formation in M2-polarized macrophages. Antioxid Redox Signal 21: 2483-2497, 2014.

167. Lakshminarasimhan $M$ and Steegborn C. Emerging mitochondrial signaling mechanisms in physiology, aging processes, and as drug targets. Exp Gerontol 46: 174-177, 2011.

168. Lampropoulou V, Sergushichev A, Bambouskova M, Nair S, Vincent EE, Loginicheva E, Cervantes-Barragan L, Ma X, Huang SC, Griss T, Weinheimer CJ, Khader S, Randolph GJ, Pearce EJ, Jones RG, Diwan A, Diamond MS, and Artyomov MN. Itaconate links inhibition of succinate dehydrogenase with macrophage metabolic remodeling and regulation of inflammation. Cell Metab 24: 158-166, 2016.

169. Lavin Y, Winter D, Blecher-Gonen R, David E, KerenShaul H, Merad M, Jung S, and Amit I. Tissue-resident macrophage enhancer landscapes are shaped by the local microenvironment. Cell 159: 1312-1326, 2014.

170. Lawrence $\mathrm{T}$ and Natoli G. Transcriptional regulation of macrophage polarization: enabling diversity with identity. Nat Rev Immunol 11: 750-761, 2011.

171. Le Moal E, Juban G, Bernard AS, Varga T, Policar C, Chazaud B, and Mounier R. Macrophage-derived 
superoxide production and antioxidant response following skeletal muscle injury. Free Radic Biol Med 120: 33-40, 2018.

172. Le Moal E, Pialoux V, Juban G, Groussard C, Zouhal H, Chazaud B, and Mounier R. Redox control of skeletal muscle regeneration. Antioxid Redox Signal 27: 276-310, 2017.

173. Lee B, Qiao L, Lu M, Yoo HS, Cheung W, Mak R, Schaack J, Feng GS, Chi NW, Olefsky JM, and Shao J. C/ EBPalpha regulates macrophage activation and systemic metabolism. Am J Physiol Endocrinol Metab 306: E1144E1154, 2014.

174. Lee HN and Surh YJ. Resolvin D1-mediated NOX2 inactivation rescues macrophages undertaking efferocytosis from oxidative stress-induced apoptosis. Biochem Pharmacol 86: 759-769, 2013.

175. Lee Y, Ka SO, Cha HN, Chae YN, Kim MK, Park SY, Bae EJ, and Park BH. Myeloid sirtuin 6 deficiency causes insulin resistance in high-fat diet-fed mice by eliciting macrophage polarization toward an M1 phenotype. Diabetes 66: 2659-2668, 2017.

176. Lee-Young RS, Hoffman NJ, Murphy KT, Henstridge DC, Samocha-Bonet D, Siebel AL, Iliades P, Zivanovic B, Hong YH, Colgan TD, Kraakman MJ, Bruce CR, Gregorevic P, McConell GK, Lynch GS, Drummond GR, Kingwell BA, Greenfield JR, and Febbraio MA. Glucose-6-phosphate dehydrogenase contributes to the regulation of glucose uptake in skeletal muscle. Mol Metab 5: 1083-1091, 2016.

177. Leiro J, Alvarez E, Arranz JA, Laguna R, Uriarte E, and Orallo F. Effects of cis-resveratrol on inflammatory murine macrophages: antioxidant activity and down-regulation of inflammatory genes. J Leukoc Biol 75: 1156-1165, 2004.

178. Lemos DR, Babaeijandaghi F, Low M, Chang CK, Lee ST, Fiore D, Zhang RH, Natarajan A, Nedospasov SA, and Rossi FM. Nilotinib reduces muscle fibrosis in chronic muscle injury by promoting TNF-mediated apoptosis of fibro/adipogenic progenitors. Nat Med 21: 786-794, 2015.

179. Leopold Wager CM, Hole CR, Wozniak KL, Olszewski MA, and Wormley FL, Jr. STAT1 signaling is essential for protection against Cryptococcus neoformans infection in mice. J Immunol 193: 4060-4071, 2014.

180. Li L, Yan B, Shi YQ, Zhang WQ, and Wen ZL. Live imaging reveals differing roles of macrophages and neutrophils during zebrafish tail fin regeneration. J Biol Chem 287: 25353-25360, 2012.

181. Li Y, Reddy MA, Miao F, Shanmugam N, Yee JK, Hawkins D, Ren B, and Natarajan R. Role of the histone H3 lysine 4 methyltransferase, SET7/9, in the regulation of NF-kappaB-dependent inflammatory genes. Relevance to diabetes and inflammation. $J$ Biol Chem 283: 2677126781, 2008.

182. Liao X, Sharma N, Kapadia F, Zhou G, Lu Y, Hong H, Paruchuri K, Mahabeleshwar GH, Dalmas E, Venteclef N, Flask CA, Kim J, Doreian BW, Lu KQ, Kaestner KH, Hamik A, Clément K, and Jain MK. Kruppel-like factor 4 regulates macrophage polarization. J Clin Invest 121: 2736-2749, 2011.

183. Linden MA, Fletcher JA, Morris EM, Meers GM, Laughlin MH, Booth FW, Sowers JR, Ibdah JA, Thyfault $\mathrm{JP}$, and Rector RS. Treating NAFLD in OLETF rats with vigorous-intensity interval exercise training. Med Sci Sports Exerc 47: 556-567, 2015.

184. Linden MA, Pincu Y, Martin SA, Woods JA, and Baynard T. Moderate exercise training provides modest protection against adipose tissue inflammatory gene expression in response to high-fat feeding. Physiol Rep 2: e12071, 2014.

185. Littlewood-Evans A, Sarret S, Apfel V, Loesle P, Dawson J, Zhang J, Muller A, Tigani B, Kneuer R, Patel S, Valeaux S, Gommermann N, Rubic-Schneider T, Junt T, and Carballido JM. GPR91 senses extracellular succinate released from inflammatory macrophages and exacerbates rheumatoid arthritis. J Exp Med 213: 1655-1662, 2016.

186. Liu TF, Vachharajani VT, Yoza BK, and McCall CE. NAD ${ }^{+}$. dependent sirtuin 1 and 6 proteins coordinate a switch from glucose to fatty acid oxidation during the acute inflammatory response. J Biol Chem 287: 25758-25769, 2012.

187. Lo HM, Chen CL, Yang CM, Wu PH, Tsou CJ, Chiang $\mathrm{KW}$, and $\mathrm{Wu} \mathrm{WB}$. The carotenoid lutein enhances matrix metalloproteinase-9 production and phagocytosis through intracellular ROS generation and ERK1/2, p38 MAPK, and RARbeta activation in murine macrophages. J Leukoc Biol 93: 723-735, 2013.

188. Lo Sasso G, Menzies KJ, Mottis A, Piersigilli A, Perino A, Yamamoto H, Schoonjans K, and Auwerx J. SIRT2 deficiency modulates macrophage polarization and susceptibility to experimental colitis. PLoS One 9: e103573, 2014.

189. Locati M, Mantovani A, and Sica A. Macrophage activation and polarization as an adaptive component of innate immunity. Adv Immunol 120: 163-184, 2013.

190. Londhe P and Davie JK. Interferon-gamma resets muscle cell fate by stimulating the sequential recruitment of JARID2 and PRC2 to promoters to repress myogenesis. Sci Signal 6: ra107, 2013.

191. Lu H, Huang D, Saederup N, Charo IF, Ransohoff RM, and Zhou L. Macrophages recruited via CCR2 produce insulin-like growth factor-1 to repair acute skeletal muscle injury. FASEB J 25: 358-369, 2011.

192. Ma EH, Bantug G, Griss T, Condotta S, Johnson RM, Samborska B, Mainolfi N, Suri V, Guak H, Balmer ML, Verway MJ, Raissi TC, Tsui H, Boukhaled G, Henriques da Costa S, Frezza C, Krawczyk CM, Friedman A, Manfredi M, Richer MJ, Hess C, and Jones RG. Serine is an essential metabolite for effector T cell expansion. Cell Metab 25: 345-357, 2017.

193. Macpherson RE, Huber JS, Frendo-Cumbo S, Simpson JA, and Wright DC. Adipose tissue insulin action and IL-6 signaling after exercise in obese mice. Med Sci Sports Exerc 47: 2034-2042, 2015.

194. Mann CJ, Perdiguero E, Kharraz Y, Aguilar S, Pessina P, Serrano AL, and Muñoz-Cánoves P. Aberrant repair and fibrosis development in skeletal muscle. Skelet Muscle 1: 21, 2011.

195. Mantovani A, Sica A, Sozzani S, Allavena P, Vecchi A, and Locati M. The chemokine system in diverse forms of macrophage activation and polarization. Trends Immunol 25: 677-686, 2004.

196. Mantovani A, Sozzani S, Locati M, Allavena P, and Sica A. Macrophage polarization: tumor-associated macrophages as a paradigm for polarized M2 mononuclear phagocytes. Trends Immunol 23: 549-555, 2002.

197. Martinez CO, McHale MJ, Wells JT, Ochoa O, Michalek JE, McManus LM, and Shireman PK. Regulation of skeletal muscle regeneration by CCR2-activating chemokines is directly related to macrophage recruitment. Am J Physiol Regul Integr Comp Physiol 299: R832-R842, 2010.

198. Martinez FO and Gordon S. The M1 and M2 paradigm of macrophage activation: time for reassessment. F1000 Prime Rep 6: 13, 2014. 
199. Martinez FO, Gordon S, Locati M, and Mantovani A. Transcriptional profiling of the human monocyte-tomacrophage differentiation and polarization: new molecules and patterns of gene expression. J Immunol 177: 7303-7311, 2006.

200. Martinez FO, Helming L, Milde R, Varin A, Melgert BN, Draijer C, Thomas B, Fabbri M, Crawshaw A, Ho LP, Ten Hacken NH, Cobos Jiménez V, Kootstra NA, Hamann J, Greaves DR, Locati M, Mantovani A, and Gordon S. Genetic programs expressed in resting and IL-4 alternatively activated mouse and human macrophages: similarities and differences. Blood 121: e57-e69, 2013.

201. Martinez FO, Sica A, Mantovani A, and Locati M. Macrophage activation and polarization. Front Biosci 13: 453461, 2008.

202. Masoodi M, Kuda O, Rossmeisl M, Flachs P, and Kopecky J. Lipid signaling in adipose tissue: connecting inflammation \& metabolism. Biochim Biophys Acta 1851: 503-518, 2015.

203. Mathieu J, Zhang Z, Zhou W, Wang AJ, Heddleston JM, Pinna CM, Hubaud A, Stadler B, Choi M, Bar M, Tewari M, Liu A, Vessella R, Rostomily R, Born D, Horwitz M, Ware C, Blau CA, Cleary MA, Rich JN, and RuoholaBaker H. HIF induces human embryonic stem cell markers in cancer cells. Cancer Res 71: 4640-4652, 2011.

204. Matsuura T, Li Y, Giacobino JP, Fu FH, and Huard J. Skeletal muscle fiber type conversion during the repair of mouse soleus: potential implications for muscle healing after injury. J Orthop Res 25: 1534-1540, 2007.

205. Mauro A. Satellite cell of skeletal muscle fibers. J Biophys Biochem Cytol 9: 493-495, 1961.

206. McGettrick AF and O'Neill LA. How metabolism generates signals during innate immunity and inflammation. $J$ Biol Chem 288: 22893-22898, 2013.

207. Meiser J, Krämer L, Sapcariu SC, Battello N, Ghelfi J, D'Herouel AF, Skupin A, and Hiller K. Pro-inflammatory macrophages sustain pyruvate oxidation through pyruvate dehydrogenase for the synthesis of itaconate and to enable cytokine expression. J Biol Chem 291: 3932-3946, 2016.

208. Ménégaut L, Thomas C, Lagrost L, and Masson D. Fatty acid metabolism in macrophages: a target in cardiometabolic diseases. Curr Opin Lipidol 28: 19-26, 2017.

209. Michelucci A, Cordes T, Ghelfi J, Pailot A, Reiling N, Goldmann O, Binz T, Wegner A, Tallam A, Rausell A, Buttini M, Linster CL, Medina E, Balling R, and Hiller K. Immune-responsive gene 1 protein links metabolism to immunity by catalyzing itaconic acid production. Proc Natl Acad Sci U S A 110: 7820-7825, 2013.

210. Mildner A, Schönheit J, Giladi A, David E, Lara-Astiaso D, Lorenzo-Vivas E, Paul F, Chappell-Maor L, Priller J, Leutz A, Amit I, and Jung S. Genomic characterization of murine monocytes reveals $\mathrm{C} / \mathrm{EBPb}$ ta transcription factor dependence of Ly6C ${ }^{-}$cells. Immunity 46: 849-862.e7, 2017.

211. Millet P, Vachharajani V, McPhail L, Yoza B, and McCall CE. GAPDH binding to TNF-alpha mRNA contributes to posttranscriptional repression in monocytes: a novel mechanism of communication between inflammation and metabolism. J Immunol 196: 2541-2551, 2016.

212. Mills CD. M1 and M2 macrophages: oracles of health and disease. Crit Rev Immunol 32: 463-488, 2012.

213. Mills CD. Anatomy of a discovery: $\mathrm{m} 1$ and $\mathrm{m} 2$ macrophages. Front Immunol 6: 212, 2015.

214. Mills EL, Kelly B, Logan A, Costa ASH, Varma M, Bryant CE, Tourlomousis P, Däbritz JHM, Gottlieb E,
Latorre I, Corr SC, McManus G, Ryan D, Jacobs HT, Szibor M, Xavier RJ, Braun T, Frezza C, Murphy MP, and O'Neill LA. Succinate dehydrogenase supports metabolic repurposing of mitochondria to drive inflammatory macrophages. Cell 167: 457-470.e13, 2016.

215. Minari AL, Oyama LM, and Dos Santos RV. Downhill exercise-induced changes in gene expression related with macrophage polarization and myogenic cells in the triceps long head of rats. Inflammation 38: 209-217, 2015.

216. Minetti GC, Colussi C, Adami R, Serra C, Mozzetta C, Parente V, Fortuni S, Straino S, Sampaolesi M, Di Padova M, Illi B, Gallinari P, Steinkühler C, Capogrossi MC, Sartorelli V, Bottinelli R, Gaetano C, and Puri PL. Functional and morphological recovery of dystrophic muscles in mice treated with deacetylase inhibitors. Nat Med 12: 1147-1150, 2006.

217. Modolell M, Corraliza IM, Link F, Soler G, and Eichmann $\mathrm{K}$. Reciprocal regulation of the nitric oxide synthase/arginase balance in mouse bone marrow-derived macrophages by TH1 and TH2 cytokines. Eur J Immunol 25: 1101-1104, 1995.

218. Mojumdar K, Liang F, Giordano C, Lemaire C, Danialou G, Okazaki T, Bourdon J, Rafei M, Galipeau J, Divangahi $\mathrm{M}$, and Petrof BJ. Inflammatory monocytes promote progression of Duchenne muscular dystrophy and can be therapeutically targeted via CCR2. EMBO Mol Med 6: 1476-1492, 2014.

219. Molinari F, Malara N, Mollace V, Rosano G, and Ferraro E. Animal models of cardiac cachexia. Int J Cardiol 219: 105-110, 2016.

220. Montagna C, Di Giacomo G, Rizza S, Cardaci S, Ferraro E, Grumati P, De Zio D, Maiani E, Muscoli C, Lauro F, Ilari S, Bernardini S, Cannata S, Gargioli C, Ciriolo MR, Cecconi F, Bonaldo P, and Filomeni G. S-nitrosoglutathione reductase deficiency-induced S-nitrosylation results in neuromuscular dysfunction. Antioxid Redox Signal 21: 570-587, 2014.

221. Mookerjee SA and Brand MD. Measurement and analysis of extracellular acid production to determine glycolytic rate. J Vis Exp e53464, 2015. DOI: 10.3791/53464.

222. Morris AC, Beresford GW, Mooney MR, and Boss JM. Kinetics of a gamma interferon response: expression and assembly of CIITA promoter IV and inhibition by methylation. Mol Cell Biol 22: 4781-4791, 2002.

223. Mortha A and Burrows K. Cytokine networks between innate lymphoid cells and myeloid cells. Front Immunol 9: 191, 2018.

224. Mosser DM and Edwards JP. Exploring the full spectrum of macrophage activation. Nat Rev Immunol 8: 958-969, 2008.

225. Motohashi N and Asakura A. Muscle satellite cell heterogeneity and self-renewal. Front Cell Dev Biol 2: 1, 2014.

226. Mounier R, Théret M, Arnold L, Cuvellier S, Bultot L, Göransson O, Sanz N, Ferry A, Sakamoto K, Foretz M, Viollet B, and Chazaud B. AMPKalpha1 regulates macrophage skewing at the time of resolution of inflammation during skeletal muscle regeneration. Cell Metab 18: 251-264, 2013.

227. Mourikis P, Sambasivan R, Castel D, Rocheteau P, Bizzarro $\mathrm{V}$, and Tajbakhsh $\mathrm{S}$. A critical requirement for notch signaling in maintenance of the quiescent skeletal muscle stem cell state. Stem Cells 30: 243-252, 2012.

228. Mozzetta C, Consalvi S, Saccone V, Tierney M, Diamantini A, Mitchell KJ, Marazzi G, Borsellino G, Battistini L, Sassoon D, Sacco A, and Puri PL. Fibroadipogenic progenitors mediate the ability of HDAC inhibitors to 
promote regeneration in dystrophic muscles of young, but not old Mdx mice. EMBO Mol Med 5: 626-639, 2013.

229. Müllebner A, Dorighello GG, Kozlov AV, and Duvigneau JC. Interaction between mitochondrial reactive oxygen species, heme oxygenase, and nitric oxide synthase stimulates phagocytosis in macrophages. Front Med (Lausanne) 4: 252, 2017.

230. Mullican SE, Gaddis CA, Alenghat T, Nair MG, Giacomin PR, Everett LJ, Feng D, Steger DJ, Schug J, Artis D, and Lazar MA. Histone deacetylase 3 is an epigenomic brake in macrophage alternative activation. Genes Dev 25: 2480-2488, 2011.

231. Munder M, Eichmann K, and Modolell M. Alternative metabolic states in murine macrophages reflected by the nitric oxide synthase/arginase balance: competitive regulation by CD4+ $\mathrm{T}$ cells correlates with Th1/Th2 phenotype. J Immunol 160: 5347-5354, 1998.

232. Muñoz-Cánoves P and Serrano AL. Macrophages decide between regeneration and fibrosis in muscle. Trends Endocrinol Metab 26: 449-450, 2015.

233. Murray PJ. Amino acid auxotrophy as a system of immunological control nodes. Nat Immunol 17: 132-139, 2016.

234. Murray PJ, Allen JE, Biswas SK, Fisher EA, Gilroy DW, Goerdt S, Gordon S, Hamilton JA, Ivashkiv LB, Lawrence T, Locati M, Mantovani A, Martinez FO, Mege JL, Mosser DM, Natoli G, Saeij JP, Schultze JL, Shirey KA, Sica A, Suttles J, Udalova I, van Ginderachter JA, Vogel SN, and Wynn TA. Macrophage activation and polarization: nomenclature and experimental guidelines. Immunity 41: 1420, 2014.

235. Musarò A, McCullagh KJ, Naya FJ, Olson EN, and Rosenthal N. IGF-1 induces skeletal myocyte hypertrophy through calcineurin in association with GATA-2 and NFATc1. Nature 400: 581-585, 1999.

236. Nagy C, Haschemi A. Time and demand are two critical dimensions of immunometabolism: the process of macrophage activation and the pentose phosphate pathway. Front Immunol 6: 164, 2015.

237. Namgaladze D and Brüne B. Fatty acid oxidation is dispensable for human macrophage IL-4-induced polarization. Biochim Biophys Acta 1841: 1329-1335, 2014.

238. Namgaladze D and Brüne B. Macrophage fatty acid oxidation and its roles in macrophage polarization and fatty acid-induced inflammation. Biochim Biophys Acta 1861: 1796-1807, 2016.

239. Nath N, Khan M, Paintlia MK, Singh I, Hoda MN, and Giri S. Metformin attenuated the autoimmune disease of the central nervous system in animal models of multiple sclerosis. J Immunol 182: 8005-8014, 2009.

240. Nath N, Khan M, Rattan R, Mangalam A, Makkar RS, de Meester C, Bertrand L, Singh I, Chen Y, Viollet B, and Giri S. Loss of AMPK exacerbates experimental autoimmune encephalomyelitis disease severity. Biochem Biophys Res Commun 386: 16-20, 2009.

241. Newsholme P, Curi R, Gordon S, and Newsholme EA. Metabolism of glucose, glutamine, long-chain fatty acids and ketone bodies by murine macrophages. Biochem $J$ 239: 121-125, 1986.

242. Newsholme P, Gordon S, and Newsholme EA. Rates of utilization and fates of glucose, glutamine, pyruvate, fatty acids and ketone bodies by mouse macrophages. Biochem J 242: 631-636, 1987.

243. Nie M, Liu J, Yang Q, Seok HY, Hu X, Deng ZL, and Wang DZ. MicroRNA-155 facilitates skeletal muscle re- generation by balancing pro- and anti-inflammatory macrophages. Cell Death Dis 7: e2261, 2016.

244. Nomura M, Liu J, Rovira II, Gonzalez-Hurtado E, Lee J, Wolfgang MJ, and Finkel T. Fatty acid oxidation in macrophage polarization. Nat Immunol 17: 216-217, 2016.

245. Novak ML, Weinheimer-Haus EM, and Koh TJ. Macrophage activation and skeletal muscle healing following traumatic injury. J Pathol 232: 344-355, 2014.

246. O'Neill LA, Kishton RJ, and Rathmell J. A guide to immunometabolism for immunologists. Nat Rev Immunol 16: 553-565, 2016.

247. Ochoa O, Sun D, Reyes-Reyna SM, Waite LL, Michalek JE, McManus LM, and Shireman PK. Delayed angiogenesis and VEGF production in CCR2-/- mice during impaired skeletal muscle regeneration. Am J Physiol Regul Integr Comp Physiol 293: R651-R661, 2007.

248. Ockenfels HM, Schultewolter T, Ockenfels G, Funk R, and Goos M. The antipsoriatic agent dimethylfumarate immunomodulates T-cell cytokine secretion and inhibits cytokines of the psoriatic cytokine network. $\mathrm{Br} J \mathrm{Der}$ matol 139: 390-395, 1998.

249. Odegaard JI, Ricardo-Gonzalez RR, Goforth MH, Morel CR, Subramanian V, Mukundan L, Red Eagle A, Vats D, Brombacher F, Ferrante AW, and Chawla A. Macrophagespecific PPARgamma controls alternative activation and improves insulin resistance. Nature 447: 1116-1120, 2007.

250. Ohtsu A, Shibutani Y, Seno K, Iwata H, Kuwayama T, and Shirasuna K. Advanced glycation end products and lipopolysaccharides stimulate interleukin-6 secretion via the RAGE/TLR4-NF-kB-ROS pathways and resveratrol attenuates these inflammatory responses in mouse macrophages. Exp Ther Med 14: 4363-4370, 2017.

251. Olenchock BA, Rathmell JC, and Vander Heiden MG. Biochemical underpinnings of immune cell metabolic phenotypes. Immunity 46: 703-713, 2017.

252. Oliveira AG, Araujo TG, Carvalho BM, Guadagnini D, Rocha GZ, Bagarolli RA, Carvalheira JB, and Saad MJ. Acute exercise induces a phenotypic switch in adipose tissue macrophage polarization in diet-induced obese rats. Obesity (Silver Spring) 21: 2545-2556, 2013.

253. Ouimet M, Ediriweera HN, Gundra UM, Sheedy FJ, Ramkhelawon B, Hutchison SB, Rinehold K, van Solingen C, Fullerton MD, Cecchini K, Rayner KJ, Steinberg GR, Zamore PD, Fisher EA, Loke P, and Moore KJ. MicroRNA-33-dependent regulation of macrophage metabolism directs immune cell polarization in atherosclerosis. J Clin Invest 125: 4334-4348, 2015.

254. Owens DJ, Sharples AP, Polydorou I, Alwan N, Donovan T, Tang J, Fraser WD, Cooper RG, Morton JP, Stewart C, and Close GL. A systems-based investigation into vitamin $\mathrm{D}$ and skeletal muscle repair, regeneration, and hypertrophy. Am J Physiol Endocrinol Metab 309: E1019-E1031, 2015.

255. Palacios D, Mozzetta C, Consalvi S, Caretti G, Saccone V, Proserpio V, Marquez VE, Valente S, Mai A, Forcales SV, Sartorelli V, and Puri PL. TNF/p38 $\alpha /$ polycomb signaling to Pax7 locus in satellite cells links inflammation to the epigenetic control of muscle regeneration. Cell Stem Cell 7: 455-469, 2010.

256. Palsson-McDermott EM, Curtis AM, Goel G, Lauterbach MA, Sheedy FJ, Gleeson LE, van den Bosch MW, Quinn SR, Domingo-Fernandez R, Johnston DG, Jiang JK, Israelsen WJ, Keane J, Thomas C, Clish C, 
Vander Heiden M, Xavier RJ, and O'Neill LA. Pyruvate kinase M2 regulates Hif-1alpha activity and IL-1beta induction and is a critical determinant of the warburg effect in LPS-activated macrophages. Cell Metab 21: 65-80, 2015.

257. Papadakis KA, Krempski J, Svingen P, Xiong Y, Sarmento OF, Lomberk GA, Urrutia RA, and Faubion WA. Kruppel-like factor KLF10 deficiency predisposes to colitis through colonic macrophage dysregulation. Am J Physiol Gastrointest Liver Physiol 309: G900-G909, 2015.

258. Pararasa C, Bailey C, and Griffiths H. Macrophage polarisation by fatty acids is PPARgamma-dependent. Free Radic Biol Med 75(Suppl 1): S31-S32, 2014.

259. Pararasa C, Ikwuobe J, Shigdar S, Boukouvalas A, Nabney IT, Brown JE, Devitt A, Bailey CJ, Bennett SJ, and Griffiths HR. Age-associated changes in long-chain fatty acid profile during healthy aging promote pro-inflammatory monocyte polarization via PPAR $\gamma$. Aging Cell 15: 128-139, 2016.

260. Park S, Won JH, Hwang I, Hong S, Lee HK, and Yu JW. Defective mitochondrial fission augments NLRP3 inflammasome activation. Sci Rep 5: 15489, 2015.

261. Patsalos A, Pap A, Varga T, Trencsenyi G, Contreras GA, Garai I, Papp Z, Dezso B, Pintye E, and Nagy L. In situ macrophage phenotypic transition is affected by altered cellular composition prior to acute sterile muscle injury. J Physiol 595: 5815-5842, 2017.

262. Pawate S, Shen Q, Fan F, and Bhat NR. Redox regulation of glial inflammatory response to lipopolysaccharide and interferongamma. J Neurosci Res 77: 540-551, 2004.

263. Pearce EL and Pearce EJ. Metabolic pathways in immune cell activation and quiescence. Immunity 38: 633-643, 2013.

264. Pearce EL, Poffenberger MC, Chang CH, and Jones RG. Fueling immunity: insights into metabolism and lymphocyte function. Science 342: 1242454, 2013. DOI: 10.1126/ science.1242454.

265. Pedersen BK and Febbraio MA. Muscles, exercise and obesity: skeletal muscle as a secretory organ. Nat Rev Endocrinol 8: 457-465, 2012.

266. Penna F, Camperi A, Muscaritoli M, Filigheddu N, and Costelli P. The role of vitamin D in cancer cachexia. Curr Opin Support Palliat Care 11: 287-292, 2017.

267. Perandini LA, Chimin P, Lutkemeyer DDS, and Câmara NOS. Chronic inflammation in skeletal muscle impairs satellite cells function during regeneration: can physical exercise restore the satellite cell niche? FEBS $J$ 285: 1973-1984, 2018.

268. Perdiguero E, Sousa-Victor P, Ruiz-Bonilla V, Jardí M, Caelles C, Serrano AL, and Muñoz-Cánoves P. p38/MKP1-regulated AKT coordinates macrophage transitions and resolution of inflammation during tissue repair. J Cell Biol 195: 307-322, 2011.

269. Perreault C, Pelletier M, Belanger R, Boileau J, Bonny Y, David M, Gyger M, Landry D, and Montplaisir S. Persistence of host Langerhans cells following allogeneic bone marrow transplantation: possible relationship with acute graft-versushost disease. Br J Haematol 60: 253-260, 1985.

270. Perrier P, Martinez FO, Locati M, Bianchi G, Nebuloni M, Vago G, Bazzoni F, Sozzani S, Allavena P, and Mantovani A. Distinct transcriptional programs activated by interleukin-10 with or without lipopolysaccharide in dendritic cells: induction of the B cell-activating chemokine, CXC chemokine ligand 13. J Immunol 172: 70317042, 2004.
271. Persichini T, Mazzone V, Polticelli F, Moreno S, Venturini G, Clementi E, and Colasanti M. Mitochondrial type I nitric oxide synthase physically interacts with cytochrome c oxidase. Neurosci Lett 384: 254-259, 2005.

272. Pesce JT, Ramalingam TR, Mentink-Kane MM, Wilson MS, El Kasmi KC, Smith AM, Thompson RW, Cheever AW, Murray PJ, and Wynn TA. Arginase-1-expressing macrophages suppress Th2 cytokine-driven inflammation and fibrosis. PLoS Pathog 5: e1000371, 2009.

273. Pessina P, Kharraz Y, Jardí M, Fukada S, Serrano AL, Perdiguero E, and Muñoz-Cánoves P. Fibrogenic cell plasticity blunts tissue regeneration and aggravates muscular dystrophy. Stem Cell Rep 4: 1046-1060, 2015.

274. Piccoli M, Urbani L, Alvarez-Fallas ME, Franzin C, Dedja A, Bertin E, Zuccolotto G, Rosato A, Pavan P, Elvassore $\mathrm{N}$, De Coppi $\mathrm{P}$, and Pozzobon M. Improvement of diaphragmatic performance through orthotopic application of decellularized extracellular matrix patch. Biomaterials 74: 245-255, 2016.

275. Pillon NJ, Bilan PJ, Fink LN, and Klip A. Cross-talk between skeletal muscle and immune cells: muscle-derived mediators and metabolic implications. Am J Physiol Endocrinol Metab 304: E453-E465, 2013.

276. Porta C, Rimoldi M, Raes G, Brys L, Ghezzi P, Di Liberto D, Dieli F, Ghisletti S, Natoli G, De Baetselier P, Mantovani A, and Sica A. Tolerance and M2 (alternative) macrophage polarization are related processes orchestrated by p50 nuclear factor kappaB. Proc Natl Acad Sci U S A 106: 14978-14983, 2009.

277. Qin L, Liu Y, Wang T, Wei SJ, Block ML, Wilson B, Liu B, and Hong JS. NADPH oxidase mediates lipopolysaccharideinduced neurotoxicity and proinflammatory gene expression in activated microglia. J Biol Chem 279: 1415-1421, 2004.

278. Qiu X, Liu S, Zhang H, Zhu B, Su Y, Zheng C, Tian R, Wang M, Kuang H, Zhao X, and Jin Y. Mesenchymal stem cells and extracellular matrix scaffold promote muscle regeneration by synergistically regulating macrophage polarization toward the M2 phenotype. Stem Cell Res Ther 9: 88, 2018.

279. Rahman I, Marwick J, and Kirkham P. Redox modulation of chromatin remodeling: impact on histone acetylation and deacetylation, NF-kappaB and pro-inflammatory gene expression. Biochem Pharmacol 68: 1255-1267, 2004.

280. Rath M, Müller I, Kropf P, Closs EI, and Munder M. Metabolism via arginase or nitric oxide synthase: two competing arginine pathways in macrophages. Front Immunol 5: 532, 2014.

281. Raza H, John A, and Shafarin J. NAC attenuates LPSinduced toxicity in aspirin-sensitized mouse macrophages via suppression of oxidative stress and mitochondrial dysfunction. PLoS One 9: e103379, 2014.

282. Rigamonti E, Zordan P, Sciorati C, Rovere-Querini P, and Brunelli S. Macrophage plasticity in skeletal muscle repair. Biomed Res Int 2014: 560629, 2014.

283. Riuzzi F, Beccafico S, Sagheddu R, Chiappalupi S, Giambanco I, Bereshchenko O, Riccardi C, Sorci G, and Donato R. Levels of S100B protein drive the reparative process in acute muscle injury and muscular dystrophy. Sci Rep 7: 12537, 2017.

284. Rodeheffer MS. Tipping the scale: muscle versus fat. Nat Cell Biol 12: 102-104, 2010.

285. Rodríguez-Prados JC, Través PG, Cuenca J, Rico D, Aragonés J, Martín-Sanz P, Cascante M, and Boscá L. 
Substrate fate in activated macrophages: a comparison between innate, classic, and alternative activation. $J \mathrm{Im}$ munol 185: 605-614, 2010.

286. Rommel C, Bodine SC, Clarke BA, Rossman R, Nunez L, Stitt TN, Yancopoulos GD, and Glass DJ. Mediation of IGF-1-induced skeletal myotube hypertrophy by PI(3)K/ Akt/mTOR and PI(3)K/Akt/GSK3 pathways. Nat Cell Biol 3: 1009-1013, 2001.

287. Röszer T. Understanding the mysterious M2 macrophage through activation markers and effector mechanisms. Mediators Inflamm 2015: 816460, 2015.

288. Rowlands DJ, Islam MN, Das SR, Huertas A, Quadri SK, Horiuchi K, Inamdar N, Emin MT, Lindert J, Ten VS, Bhattacharya S, and Bhattacharya J. Activation of TNFR1 ectodomain shedding by mitochondrial $\mathrm{Ca} 2+$ determines the severity of inflammation in mouse lung microvessels. J Clin Invest 121: 1986-1999, 2011.

289. Rubartelli A, Gattorno M, Netea MG, and Dinarello CA. Interplay between redox status and inflammasome activation. Trends Immunol 32: 559-566, 2011.

290. Rubic T, Lametschwandtner G, Jost S, Hinteregger S, Kund J, Carballido-Perrig N, Schwärzler C, Junt T, Voshol H, Meingassner JG, Mao X, Werner G, Rot A, and Carballido JM. Triggering the succinate receptor GPR91 on dendritic cells enhances immunity. Nat Immunol 9: 1261-1269, 2008.

291. Ruffell D, Mourkioti F, Gambardella A, Kirstetter P, Lopez RG, Rosenthal N, and Nerlov C. A CREB-C/ EBPbeta cascade induces M2 macrophage-specific gene expression and promotes muscle injury repair. Proc Natl Acad Sci U S A 106: 17475-17480, 2009.

292. Rutledge KE, Cheng Q, and Jabbarzadeh E. Modulation of inflammatory response and induction of bone formation based on combinatorial effects of resveratrol. J Nanomed Nanotechnol 7: 350, 2016.

293. Ryall JG, Dell'Orso S, Derfoul A, Juan A, Zare H, Feng X, Clermont D, Koulnis M, Gutierrez-Cruz G, Fulco M, and Sartorelli V. The NAD(+)-dependent SIRT1 deacetylase translates a metabolic switch into regulatory epigenetics in skeletal muscle stem cells. Cell Stem Cell 16: 171-183, 2015.

294. Rybalko V, Hsieh PL, Merscham-Banda M, Suggs LJ, and Farrar RP. The development of macrophage-mediated cell therapy to improve skeletal muscle function after injury. PLoS One 10: e0145550, 2015.

295. Saccone V, Consalvi S, Giordani L, Mozzetta C, Barozzi I, Sandoná M, Ryan T, Rojas-Muñoz A, Madaro L, Fasanaro $P$, Borsellino G, De Bardi M, Frigè G, Termanini A, Sun X, Rossant J, Bruneau BG, Mercola M, Minucci S, and Puri PL. HDAC-regulated myomiRs control BAF60 variant exchange and direct the functional phenotype of fibro-adipogenic progenitors in dystrophic muscles. Genes Dev 28: 841-857, 2014.

296. Saclier M, Cuvellier S, Magnan M, Mounier R, and Chazaud B. Monocyte/macrophage interactions with myogenic precursor cells during skeletal muscle regeneration. FEBS J 280: 4118-4130, 2013.

297. Saclier M, Yacoub-Youssef H, Mackey AL, Arnold L, Ardjoune H, Magnan M, Sailhan F, Chelly J, Pavlath GK, Mounier R, Kjaer M, and Chazaud B. Differentially activated macrophages orchestrate myogenic precursor cell fate during human skeletal muscle regeneration. Stem Cells 31: 384-396, 2013.
298. Sadtler K, Estrellas K, Allen BW, Wolf MT, Fan H, Tam AJ, Patel CH, Luber BS, Wang H, Wagner KR, Powell JD, Housseau F, Pardoll DM, and Elisseeff JH. Developing a pro-regenerative biomaterial scaffold microenvironment requires T helper 2 cells. Science 352: 366-370, 2016.

299. Sager HB, Hulsmans M, Lavine KJ, Moreira MB, Heidt T, Courties G, Sun Y, Iwamoto Y, Tricot B, Khan OF, Dahlman JE, Borodovsky A, Fitzgerald K, Anderson DG, Weissleder R, Libby P, Swirski FK, and Nahrendorf M. Proliferation and recruitment contribute to myocardial macrophage expansion in chronic heart failure. Circ Res 119: 853-864, 2016.

300. San Emeterio CL, Olingy CE, Chu Y, and Botchwey EA. Selective recruitment of non-classical monocytes promotes skeletal muscle repair. Biomaterials 117: 32-43, 2017.

301. Sanmun D, Witasp E, Jitkaew S, Tyurina YY, Kagan VE, Ahlin A, Palmblad J, and Fadeel B. Involvement of a functional NADPH oxidase in neutrophils and macrophages during programmed cell clearance: implications for chronic granulomatous disease. Am J Physiol Cell Physiol 297: C621-C631, 2009.

302. Satoh M, Fujimoto S, Haruna Y, Arakawa S, Horike H, Komai N, Sasaki T, Tsujioka K, Makino H, and Kashihara N. NAD(P)H oxidase and uncoupled nitric oxide synthase are major sources of glomerular superoxide in rats with experimental diabetic nephropathy. Am J Physiol Renal Physiol 288: F1144-F1152, 2005.

303. Satoh T, Takeuchi O, Vandenbon A, Yasuda K, Tanaka Y, Kumagai Y, Miyake T, Matsushita K, Okazaki T, Saitoh T, Honma K, Matsuyama T, Yui K, Tsujimura T, Standley DM, Nakanishi K, Nakai K, and Akira S. The Jmjd3-Irf4 axis regulates M2 macrophage polarization and host responses against helminth infection. Nat Immunol 11: 936944, 2010.

304. Scheiblich $\mathrm{H}$ and Bicker G. Nitric oxide regulates antagonistically phagocytic and neurite outgrowth inhibiting capacities of microglia. Dev Neurobiol 76: 566-584, 2016.

305. Schiaffino S, Rossi AC, Smerdu V, Leinwand LA, and Reggiani C. Developmental myosins: expression patterns and functional significance. Skelet Muscle 5: 22, 2015.

306. Seale P, Sabourin LA, Girgis-Gabardo A, Mansouri A, Gruss P, and Rudnicki MA. Pax7 is required for the specification of myogenic satellite cells. Cell 102: $777-$ 786, 2000.

307. Segawa M, Fukada S, Yamamoto Y, Yahagi H, Kanematsu M, Sato M, Ito T, Uezumi A, Hayashi S, MiyagoeSuzuki Y, Takeda S, Tsujikawa K, and Yamamoto $\mathrm{H}$. Suppression of macrophage functions impairs skeletal muscle regeneration with severe fibrosis. Exp Cell Res 314: 3232-3244, 2008.

308. Semba H, Takeda N, Isagawa T, Sugiura Y, Honda K, Wake M, Miyazawa H, Yamaguchi Y, Miura M, Jenkins DM, Choi H, Kim JW, Asagiri M, Cowburn AS, Abe H, Soma K, Koyama K, Katoh M, Sayama K, Goda N, Johnson RS, Manabe I, Nagai R, and Komuro I. HIF1alpha-PDK1 axis-induced active glycolysis plays an essential role in macrophage migratory capacity. Nat Commun 7: 11635, 2016.

309. Sheikh F, Dickensheets H, Gamero AM, Vogel SN, and Donnelly RP. An essential role for IFN-beta in the induction of IFN-stimulated gene expression by LPS in macrophages. J Leukoc Biol 96: 591-600, 2014. 
310. Shirai T, Nazarewicz RR, Wallis BB, Yanes RE, Watanabe R, Hilhorst M, Tian L, Harrison DG, Giacomini JC, Assimes TL, Goronzy JJ, and Weyand CM. The glycolytic enzyme PKM2 bridges metabolic and inflammatory dysfunction in coronary artery disease. J Exp Med 213: 337354, 2016.

311. Shireman PK, Contreras-Shannon V, Ochoa O, Karia BP, Michalek JE, and McManus LM. MCP-1 deficiency causes altered inflammation with impaired skeletal muscle regeneration. J Leukoc Biol 81: 775-785, 2007.

312. Sica A, Invernizzi $P$, and Mantovani A. Macrophage plasticity and polarization in liver homeostasis and pathology. Hepatology 59: 2034-2042, 2014.

313. Sica A and Mantovani A. Macrophage plasticity and polarization: in vivo veritas. J Clin Invest 122: 787-795, 2012.

314. Sicari BM, Dziki JL, Siu BF, Medberry CJ, Dearth CL, and Badylak SF. The promotion of a constructive macrophage phenotype by solubilized extracellular matrix. Biomaterials 35: 8605-8612, 2014.

315. Silveira LS, Antunes Bde M, Minari AL, Dos Santos RV, Neto JC, and Lira FS. Macrophage polarization: implications on metabolic diseases and the role of exercise. Crit Rev Eukaryot Gene Expr 26: 115-132, 2016.

316. Song L, Papaioannou G, Zhao H, Luderer HF, Miller C, Dall'Osso C, Nazarian RM, Wagers AJ, and Demay MB. The vitamin $\mathrm{D}$ receptor regulates tissue resident macrophage response to injury. Endocrinology 157: 4066-4075, 2016.

317. Speijer D. Being right on Q: shaping eukaryotic evolution. Biochem J 473: 4103-4127, 2016.

318. Srikuea R and Hirunsai M. Effects of intramuscular administration of 1alpha,25(OH)2D3 during skeletal muscle regeneration on regenerative capacity, muscular fibrosis, and angiogenesis. J Appl Physiol (1985) 120: 1381-1393, 2016.

319. Stout RD, Jiang C, Matta B, Tietzel I, Watkins SK, and Suttles J. Macrophages sequentially change their functional phenotype in response to changes in microenvironmental influences. J Immunol 175: 342-349, 2005.

320. Strelko CL, Lu W, Dufort FJ, Seyfried TN, Chiles TC, Rabinowitz JD, and Roberts MF. Itaconic acid is a mammalian metabolite induced during macrophage activation. J Am Chem Soc 133: 16386-16389, 2011.

321. Stuehr DJ and Nathan CF. Nitric oxide. A macrophage product responsible for cytostasis and respiratory inhibition in tumor target cells. J Exp Med 169: 1543-1555, 1989.

322. Sumbayev VV. LPS-induced Toll-like receptor 4 signalling triggers cross-talk of apoptosis signal-regulating kinase 1 (ASK1) and HIF-1alpha protein. FEBS Lett 582: 319-326, 2008.

323. Sun L, Chen B, Jiang R, Li J, and Wang B. Resveratrol inhibits lung cancer growth by suppressing M2-like polarization of tumor associated macrophages. Cell Immunol 311: 86-93, 2017.

324. Tafani M, Russo A, Di Vito M, Sale P, Pellegrini L, Schito L, Gentileschi S, Bracaglia R, Marandino F, Garaci $\mathrm{E}$, and Russo MA. Up-regulation of pro-inflammatory genes as adaptation to hypoxia in MCF-7 cells and in human mammary invasive carcinoma microenvironment. Cancer Sci 101: 1014-1023, 2010.

325. Tafani M, Schito L, Pellegrini L, Villanova L, Marfe G, Anwar T, Rosa R, Indelicato M, Fini M, Pucci B, and Russo MA. Hypoxia-increased RAGE and P2X7R expression regulates tumor cell invasion through phosphorylation of Erk1/2 and Akt and nuclear translocation of NF-\{kappa\}B. Carcinogenesis 32: 1167-1175, 2011.

326. Takamura M, Kurokawa K, Ootsuji H, Inoue O, Okada H, Nomura A, Kaneko S, and Usui S. Long-term administration of eicosapentaenoic acid improves post-myocardial infarction cardiac remodeling in mice by regulating macrophage polarization. J Am Heart Assoc 6: e004560, 2017.

327. Takaoka A, Yanai H, Kondo S, Duncan G, Negishi H, Mizutani T, Kano S, Honda K, Ohba Y, Mak TW, and Taniguchi T. Integral role of IRF-5 in the gene induction programme activated by Toll-like receptors. Nature 434: 243-249, 2005.

328. Takeda N, O’Dea EL, Doedens A, Kim JW, Weidemann A, Stockmann C, Asagiri M, Simon MC, Hoffmann A, and Johnson RS. Differential activation and antagonistic function of HIF-\{alpha\} isoforms in macrophages are essential for NO homeostasis. Genes Dev 24: 491-501, 2010.

329. Talamonti E, Pauter AM, Asadi A, Fischer AW, Chiurchiù $\mathrm{V}$, and Jacobsson A. Impairment of systemic DHA synthesis affects macrophage plasticity and polarization: implications for DHA supplementation during inflammation. Cell Mol Life Sci 74: 2815-2826, 2017.

330. Talari M, Kapadia B, Kain V, Seshadri S, Prajapati B, Rajput P, Misra P, and Parsa KV. MicroRNA-16 modulates macrophage polarization leading to improved insulin sensitivity in myoblasts. Biochimie 119: 16-26, 2015.

331. Tan HY, Wang N, Li S, Hong M, Wang X, and Feng Y. The reactive oxygen species in macrophage polarization: reflecting its dual role in progression and treatment of human diseases. Oxid Med Cell Longev 2016: 2795090, 2016. DOI: $10.1155 / 2016 / 2795090$.

332. Tan Z, Xie N, Cui H, Moellering DR, Abraham E, Thannickal VJ, and Liu G. Pyruvate dehydrogenase kinase 1 participates in macrophage polarization via regulating glucose metabolism. J Immunol 194: 6082-6089, 2015.

333. Tang Y, Reay DP, Salay MN, Mi MY, Clemens PR, Guttridge DC, Robbins PD, Huard J, and Wang B. Inhibition of the IKK/NF-kB pathway by AAV gene transfer improves muscle regeneration in older $\mathrm{mdx}$ mice. Gene Ther 17: 1476-1483, 2010.

334. Tannahill GM, Curtis AM, Adamik J, Palsson-McDermott EM, McGettrick AF, Goel G, Frezza C, Bernard NJ, Kelly B, Foley NH, Zheng L, Gardet A, Tong Z, Jany SS, Corr SC, Haneklaus M, Caffrey BE, Pierce K, Walmsley S, Beasley FC, Cummins E, Nizet V, Whyte M, Taylor CT, Lin H, Masters SL, Gottlieb E, Kelly VP, Clish C, Auron $\mathrm{PE}$, Xavier RJ, and O'Neill LA. Succinate is an inflammatory signal that induces IL-1beta through HIF-1alpha. Nature 496: 238-242, 2013.

335. Tannahill GM, Iraci N, Gaude E, Frezza C, and Pluchino S. Metabolic reprograming of mononuclear phagocytes in progressive multiple sclerosis. Front Immunol 6: 106, 2015.

336. Taoudi S, Gonneau C, Moore K, Sheridan JM, Blackburn CC, Taylor E, and Medvinsky A. Extensive hematopoietic stem cell generation in the AGM region via maturation of VE-cadherin+CD45+ pre-definitive HSCs. Cell Stem Cell 3: 99-108, 2008.

337. Tattoli I, Lembo-Fazio L, Nigro G, Carneiro LA, Ferraro E, Rossi G, Martino MC, de Stefano ME, Cecconi F, Girardin SE, Philpott DJ, and Bernardini ML. Intracellular bacteriolysis triggers a massive apoptotic cell death in 
Shigella-infected epithelial cells. Microbes Infect 10: 1114-1123, 2008.

338. Tawakol A, Singh P, Mojena M, Pimentel-Santillana M, Emami H, MacNabb M, Rudd JH, Narula J, Enriquez JA, Través PG, Fernández-Velasco M, Bartrons R, Martín-Sanz P, Fayad ZA, Tejedor A, and Boscá L. HIF-1alpha and PFKFB3 mediate a tight relationship between proinflammatory activation and anerobic metabolism in atherosclerotic macrophages. Arterioscler Thromb Vasc Biol 35: 1463-1471, 2015.

339. Tedesco FS, Dellavalle A, Diaz-Manera J, Messina G, and Cossu G. Repairing skeletal muscle: regenerative potential of skeletal muscle stem cells. J Clin Invest 120: 11-19, 2010.

340. Teixeira CF, Zamunér SR, Zuliani JP, Fernandes CM, Cruz-Hofling MA, Fernandes I, Chaves F, and Gutiérrez JM. Neutrophils do not contribute to local tissue damage, but play a key role in skeletal muscle regeneration, in mice injected with Bothrops asper snake venom. Muscle Nerve 28: 449-459, 2003.

341. ter Horst EN, Hakimzadeh N, van der Laan AM, Krijnen PA, Niessen HW, and Piek JJ. Modulators of macrophage polarization influence healing of the infarcted myocardium. Int J Mol Sci 16: 29583-29591, 2015.

342. Tidball JG. Mechanisms of muscle injury, repair, and regeneration. Compr Physiol 1: 2029-2062, 2011.

343. Tidball JG and Villalta SA. Regulatory interactions between muscle and the immune system during muscle regeneration. Am J Physiol Regul Integr Comp Physiol 298: R1173-R1187, 2010.

344. Tidball JG and Wehling-Henricks M. Macrophages promote muscle membrane repair and muscle fibre growth and regeneration during modified muscle loading in mice in vivo. $J$ Physiol 578: 327-336, 2007.

345. Tocchetti CG, Carpi A, Coppola C, Quintavalle C, Rea D, Campesan M, Arcari A, Piscopo G, Cipresso C, Monti MG, De Lorenzo C, Arra C, Condorelli G, Di Lisa F, and Maurea N. Ranolazine protects from doxorubicin-induced oxidative stress and cardiac dysfunction. Eur J Heart Fail 16: 358-366, 2014.

346. Tonkin J, Temmerman L, Sampson RD, Gallego-Colon E, Barberi L, Bilbao D, Schneider MD, Musarò A, and Rosenthal N. Monocyte/macrophage-derived IGF-1 orchestrates murine skeletal muscle regeneration and modulates autocrine polarization. Mol Ther 23: 1189-1200, 2015.

347. This reference has been deleted.

348. Tonkin J, Villarroya F, Puri PL, and Vinciguerra M. SIRT1 signaling as potential modulator of skeletal muscle diseases. Curr Opin Pharmacol 12: 372-376, 2012.

349. Tsai SH, Lin-Shiau SY, and Lin JK. Suppression of nitric oxide synthase and the down-regulation of the activation of NFkappaB in macrophages by resveratrol. $\mathrm{Br} J$ Pharmacol 126: 673-680, 1999.

350. Tümer C, Bilgin HM, Obay BD, Diken H, Atmaca M, and Kelle M. Effect of nitric oxide on phagocytic activity of lipopolysaccharide-induced macrophages: possible role of exogenous L-arginine. Cell Biol Int 31: 565-569, 2007.

351. Tyurina YY, Basova LV, Konduru NV, Tyurin VA, Potapovich AI, Cai P, Bayir H, Stoyanovsky D, Pitt BR, Shvedova AA, Fadeel B, and Kagan VE. Nitrosative stress inhibits the aminophospholipid translocase resulting in phosphatidylserine externalization and macrophage engulfment: implications for the resolution of inflammation. J Biol Chem 282: 8498-8509, 2007.

352. Uezumi A, Ito T, Morikawa D, Shimizu N, Yoneda T, Segawa M, Yamaguchi M, Ogawa R, Matev MM,
Miyagoe-Suzuki Y, Takeda S, Tsujikawa K, Tsuchida K, Yamamoto H, and Fukada S. Fibrosis and adipogenesis originate from a common mesenchymal progenitor in skeletal muscle. J Cell Sci 124: 3654-3664, 2011.

353. Van den Bossche J, Baardman J, Otto NA, van der Velden $\mathrm{S}$, Neele AE, van den Berg SM, Luque-Martin R, Chen HJ, Boshuizen MC, Ahmed M, Hoeksema MA, de Vos $\mathrm{AF}$, and de Winther MP. Mitochondrial dysfunction prevents repolarization of inflammatory macrophages. Cell Rep 17: 684-696, 2016.

354. Van den Bossche J, Lamers WH, Koehler ES, Geuns JM, Alhonen L, Uimari A, Pirnes-Karhu S, Van Overmeire E, Morias Y, Brys L, Vereecke L, De Baetselier P, and Van Ginderachter JA. Pivotal advance: arginase-1-independent polyamine production stimulates the expression of IL-4induced alternatively activated macrophage markers while inhibiting LPS-induced expression of inflammatory genes. J Leukoc Biol 91: 685-699, 2012.

355. Van den Bossche J, O'Neill LA, and Menon D. Macrophage immunometabolism: where are we (going)? Trends Immunol 38: 395-406, 2017.

356. Varga T, Mounier R, Horvath A, Cuvellier S, Dumont F, Poliska S, Ardjoune H, Juban G, Nagy L, and Chazaud B. Highly dynamic transcriptional signature of distinct macrophage subsets during sterile inflammation, resolution, and tissue repair. J Immunol 196: 4771-4782, 2016.

357. Vats D, Mukundan L, Odegaard JI, Zhang L, Smith KL, Morel CR, Wagner RA, Greaves DR, Murray PJ, and Chawla A. Oxidative metabolism and PGC-1beta attenuate macrophage-mediated inflammation. Cell Metab 4: 13-24, 2006.

358. Venereau E, Casalgrandi M, Schiraldi M, Antoine DJ, Cattaneo A, De Marchis F, Liu J, Antonelli A, Preti A, Raeli L, Shams SS, Yang H, Varani L, Andersson U, Tracey KJ, Bachi A, Uguccioni M, and Bianchi ME. Mutually exclusive redox forms of HMGB1 promote cell recruitment or proinflammatory cytokine release. J Exp Med 209: 1519-1528, 2012.

359. Verdeguer F and Aouadi M. Macrophage heterogeneity and energy metabolism. Exp Cell Res 360: 35-40, 2017.

360. Vezzoli M, Castellani P, Corna G, Castiglioni A, Bosurgi L, Monno A, Brunelli S, Manfredi AA, Rubartelli A, and Rovere-Querini P. High-mobility group box 1 release and redox regulation accompany regeneration and remodeling of skeletal muscle. Antioxid Redox Signal 15: 2161-2174, 2011.

361. Vidal B, Ardite E, Suelves M, Ruiz-Bonilla V, Janué A, Flick MJ, Degen JL, Serrano AL, and Muñoz-Cánoves P. Amelioration of Duchenne muscular dystrophy in $\mathrm{mdx}$ mice by elimination of matrix-associated fibrin-driven inflammation coupled to the $\alpha \mathrm{M} \beta 2$ leukocyte integrin receptor. Hum Mol Genet 21: 1989-2004, 2012.

362. Vidal B, Serrano AL, Tjwa M, Suelves M, Ardite E, De Mori R, Baeza-Raja B, Martínez de Lagrán M, Lafuste P, Ruiz-Bonilla V, Jardí M, Gherardi R, Christov C, Dierssen M, Carmeliet P, Degen JL, Dewerchin M, and MuñozCánoves P. Fibrinogen drives dystrophic muscle fibrosis via a TGFbeta/alternative macrophage activation pathway. Genes Dev 22: 1747-1752, 2008.

363. Villalta SA, Deng B, Rinaldi C, Wehling-Henricks M, and Tidball JG. IFN-gamma promotes muscle damage in the mdx mouse model of Duchenne muscular dystrophy by suppressing M2 macrophage activation and inhibiting muscle cell proliferation. J Immunol 187: 5419-5428, 2011.

364. Villalta SA, Nguyen HX, Deng B, Gotoh T, and Tidball JG. Shifts in macrophage phenotypes and macrophage 
competition for arginine metabolism affect the severity of muscle pathology in muscular dystrophy. Hum Mol Genet 18: 482-496, 2009.

365. Villalta SA, Rinaldi C, Deng B, Liu G, Fedor B, and Tidball JG. Interleukin-10 reduces the pathology of $\mathrm{mdx}$ muscular dystrophy by deactivating M1 macrophages and modulating macrophage phenotype. Hum Mol Genet 20: 790-805, 2011.

366. Villalta SA, Rosenberg AS, and Bluestone JA. The immune system in Duchenne muscular dystrophy: friend or foe. Rare Dis 3: e1010966, 2015.

367. Villalta SA, Rosenthal W, Martinez L, Kaur A, Sparwasser T, Tidball JG, Margeta M, Spencer MJ, and Bluestone JA. Regulatory $\mathrm{T}$ cells suppress muscle inflammation and injury in muscular dystrophy. Sci Transl Med 6: 258ra142, 2014.

368. von Haehling S, Ebner N, Dos Santos MR, Springer J, and Anker SD. Muscle wasting and cachexia in heart failure: mechanisms and therapies. Nat Rev Cardiol 14: 323-341, 2017.

369. Wagatsuma A, Kotake N, and Yamada S. Muscle regeneration occurs to coincide with mitochondrial biogenesis. Mol Cell Biochem 349: 139-147, 2011.

370. Wai T and Langer T. Mitochondrial dynamics and metabolic regulation. Trends Endocrinol Metab 27: 105-117, 2016.

371. Wan J, Benkdane M, Teixeira-Clerc F, Bonnafous S, Louvet A, Lafdil F, Pecker F, Tran A, Gual P, Mallat A, Lotersztajn S, and Pavoine C. M2 Kupffer cells promote M1 Kupffer cell apoptosis: a protective mechanism against alcoholic and nonalcoholic fatty liver disease. Hepatology 59: 130-142, 2014.

372. Wang H, Melton DW, Porter L, Sarwar ZU, McManus LM, and Shireman PK. Altered macrophage phenotype transition impairs skeletal muscle regeneration. Am J Pathol 184: 1167-1184, 2014.

373. Wang N, Liang H, and Zen K. Molecular mechanisms that influence the macrophage $\mathrm{m} 1-\mathrm{m} 2$ polarization balance. Front Immunol 5: 614, 2014.

374. Wang YX and Rudnicki MA. Satellite cells, the engines of muscle repair. Nat Rev Mol Cell Biol 13: 127-133, 2011.

375. Waqas SFH, Hoang AC, Lin YT, Ampem G, Azegrouz H, Balogh L, Thuróczy J, Chen JC, Gerling IC, Nam S, Lim JS, Martinez-Ibañez J, Real JT, Paschke S, Quillet R, Ayachi S, Simonin F, Schneider EM, Brinkman JA, Lamming DW, Seroogy CM, and Röszer T. Neuropeptide FF increases M2 activation and self-renewal of adipose tissue macrophages. J Clin Invest 127: 2842-2854, 2017.

376. Warren GL, Hulderman T, Mishra D, Gao X, Millecchia L, O'Farrell L, Kuziel WA, and Simeonova PP. Chemokine receptor CCR2 involvement in skeletal muscle regeneration. FASEB J 19: 413-415, 2005.

377. Wehling-Henricks $M$ and Tidball JG. Neuronal nitric oxide synthase-rescue of dystrophin/utrophin double knockout mice does not require nNOS localization to the cell membrane. PLoS One 6: e25071, 2011.

378. Wei X, Song H, Yin L, Rizzo MG, Sidhu R, Covey DF, Ory DS, and Semenkovich CF. Fatty acid synthesis configures the plasma membrane for inflammation in diabetes. Nature 539: 294-298, 2016.

379. Wellen KE and Thompson CB. A two-way street: reciprocal regulation of metabolism and signalling. Nat Rev Mol Cell Biol 13: 270-276, 2012.

380. Wentworth JM, Naselli G, Brown WA, Doyle L, Phipson B, Smyth GK, Wabitsch M, O’Brien PE, and Harrison LC.
Pro-inflammatory CD11c+CD206+ adipose tissue macrophages are associated with insulin resistance in human obesity. Diabetes 59: 1648-1656, 2010.

381. Wilson HM. SOCS proteins in macrophage polarization and function. Front Immunol 5: 357, 2014.

382. Wynn TA, Chawla A, and Pollard JW. Macrophage biology in development, homeostasis and disease. Nature 496: 445-455, 2013.

383. Xia M, Liu J, Wu X, Liu S, Li G, Han C, Song L, Li Z, Wang Q, Wang J, Xu T, and Cao X. Histone methyltransferase Ash11 suppresses interleukin-6 production and inflammatory autoimmune diseases by inducing the ubiquitin-editing enzyme A20. Immunity 39: 470-481, 2013.

384. Xie C, Liu C, Wu B, Lin Y, Ma T, Xiong H, Wang Q, Li $\mathrm{Z}$, Ma C, and Tu Z. Effects of IRF1 and IFN-beta interaction on the M1 polarization of macrophages and its antitumor function. Int J Mol Med 38: 148-160, 2016.

385. Xin H, Wang M, Tang W, Shen Z, Miao L, Wu W, Li C, Wang X, Xin X, and Zhu YZ. Hydrogen sulfide attenuates inflammatory hepcidin by reducing IL- 6 secretion and promoting SIRT1-mediated STAT3 deacetylation. Antioxid Redox Signal 24: 70-83, 2016.

386. Xue J, Schmidt SV, Sander J, Draffehn A, Krebs W, Quester I, De Nardo D, Gohel TD, Emde M, Schmidleithner L, Ganesan H, Nino-Castro A, Mallmann MR, Labzin L, Theis H, Kraut M, Beyer M, Latz E, Freeman TC, Ulas T, and Schultze JL. Transcriptome-based network analysis reveals a spectrum model of human macrophage activation. Immunity 40: 274-288, 2014.

387. Yabumoto C, Akazawa H, Yamamoto R, Yano M, KudoSakamoto Y, Sumida T, Kamo T, Yagi H, Shimizu Y, SagaKamo A, Naito AT, Oka T, Lee JK, Suzuki J, Sakata Y, Uejima E, and Komuro I. Angiotensin II receptor blockade promotes repair of skeletal muscle through down-regulation of aging-promoting C1q expression. Sci Rep 5: 14453, 2015.

388. Yaden BC, Croy JE, Wang Y, Wilson JM, Datta-Mannan A, Shetler P, Milner A, Bryant HU, Andrews J, Dai G, and Krishnan V. Follistatin: a novel therapeutic for the improvement of muscle regeneration. J Pharmacol Exp Ther 349: 355-371, 2014.

389. Yin H, Price F, and Rudnicki MA. Satellite cells and the muscle stem cell niche. Physiol Rev 93: 23-67, 2013.

390. Yin K, You Y, Swier V, Tang L, Radwan MM, Pandya AN, and Agrawal DK. Vitamin D protects against atherosclerosis via regulation of cholesterol efflux and macrophage polarization in hypercholesterolemic swine. Arterioscler Thromb Vasc Biol 35: 2432-2442, 2015.

391. Ying H, Kang Y, Zhang H, Zhao D, Xia J, Lu Z, Wang H, $\mathrm{Xu}$ F, and Shi L. MiR-127 modulates macrophage polarization and promotes lung inflammation and injury by activating the JNK pathway. J Immunol 194: 1239-1251, 2015.

392. Ying W, Tseng A, Chang RC, Morin A, Brehm T, Triff K, Nair V, Zhuang G, Song $H$, Kanameni S, Wang $H$, Golding MC, Bazer FW, Chapkin RS, Safe S, and Zhou B. MicroRNA-223 is a crucial mediator of PPARgammaregulated alternative macrophage activation. J Clin Invest 125: 4149-4159, 2015.

393. Zani B, Cossu G, Adamo S, and Molinaro M. Biosynthetic changes in myosin heavy subunit during myogenesis in culture. Differentiation 10: 95-100, 1978.

394. Zhang J, Xiao Z, Qu C, Cui W, Wang X, and Du J. CD8 T cells are involved in skeletal muscle regeneration through facilitating MCP-1 secretion and Gr1(high) macrophage infiltration. J Immunol 193: 5149-5160, 2014. 
395. Zhang X, Zhou M, Guo Y, Song Z, and Liu B. 1,25Dihydroxyvitamin D (3) promotes high glucose-induced M1 macrophage switching to M2 via the VDR-PPAR $\gamma$ signaling pathway. Biomed Res Int 2015: 157834, 2015.

396. Zhang Y, Choksi S, Chen K, Pobezinskaya Y, Linnoila I, and Liu ZG. ROS play a critical role in the differentiation of alternatively activated macrophages and the occurrence of tumor-associated macrophages. Cell Res 23: 898-914, 2013.

397. Zhao Q, Chu Z, Zhu L, Yang T, Wang P, Liu F, Huang Y, Zhang F, Zhang X, Ding W, and Zhao Y. 2-Deoxy-dglucose treatment decreases anti-inflammatory M2 macrophage polarization in mice with tumor and allergic airway inflammation. Front Immunol 8: 637, 2017.

398. Zhou L, Porter JD, Cheng G, Gong B, Hatala DA, Merriam AP, Zhou X, Rafael JA, and Kaminski HJ. Temporal and spatial mRNA expression patterns of TGF-beta1, 2, 3 and TbetaRI, II, III in skeletal muscles of $\mathrm{mdx}$ mice. Neuromuscul Disord 16: 32-38, 2006.

399. Zhu YP, Brown JR, Sag D, Zhang L, and Suttles J. Adenosine $5^{\prime}$-monophosphate-activated protein kinase regulates IL-10-mediated anti-inflammatory signaling pathways in macrophages. J Immunol 194: 584-594, 2015.

400. Zordan P, Rigamonti E, Freudenberg K, Conti V, Azzoni E, Rovere-Querini P, and Brunelli S. Macrophages commit postnatal endothelium-derived progenitors to angiogenesis and restrict endothelial to mesenchymal transition during muscle regeneration. Cell Death Dis 5: e1031, 2014.

Address correspondence to: Dr. Elisabetta Ferraro Laboratory of Pathophysiology of Cachexia and Metabolism of Skeletal Muscle IRCCS San Raffaele Pisana Via di Val Cannuta 247 Rome 00166 Italy

E-mail: elisabetta.ferraro@ymail.com

Date of first submission to ARS Central, October 22, 2017; date of final revised submission, July 25, 2018; date of acceptance, August 1, 2018.

\begin{tabular}{|c|}
\hline 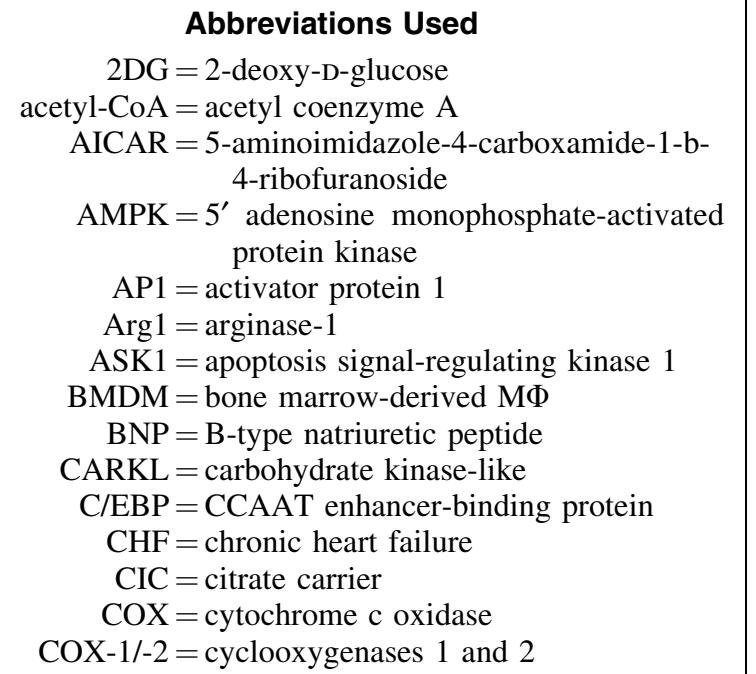 \\
\hline
\end{tabular}

\begin{tabular}{|c|}
\hline 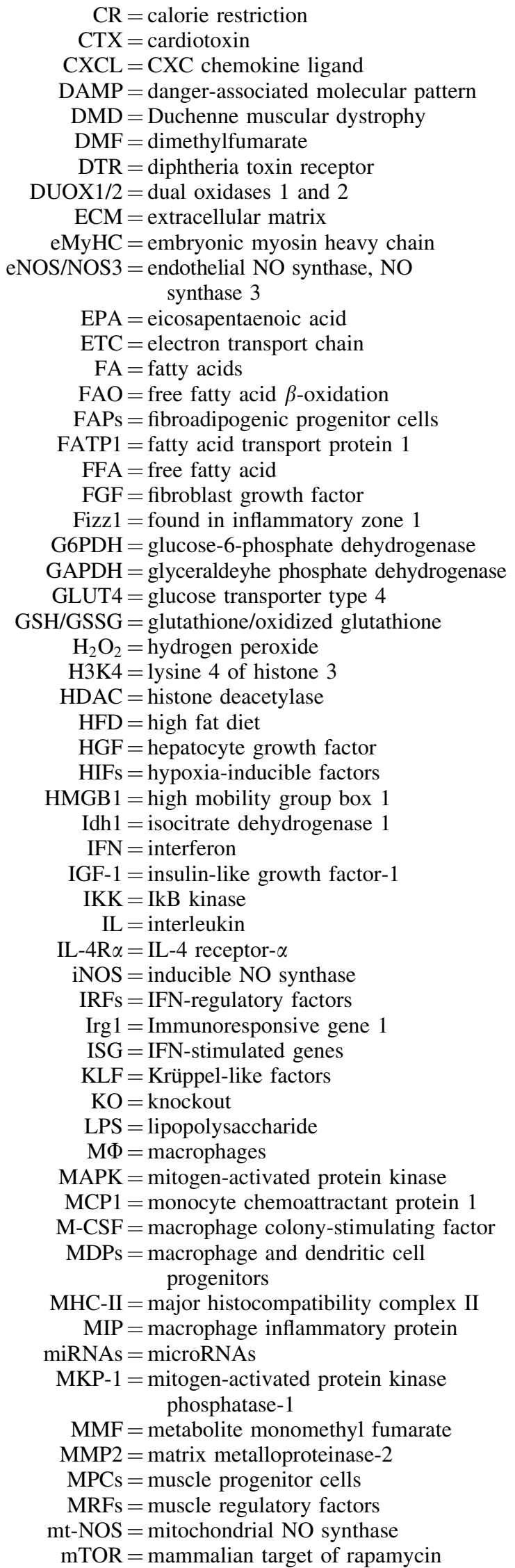 \\
\hline
\end{tabular}




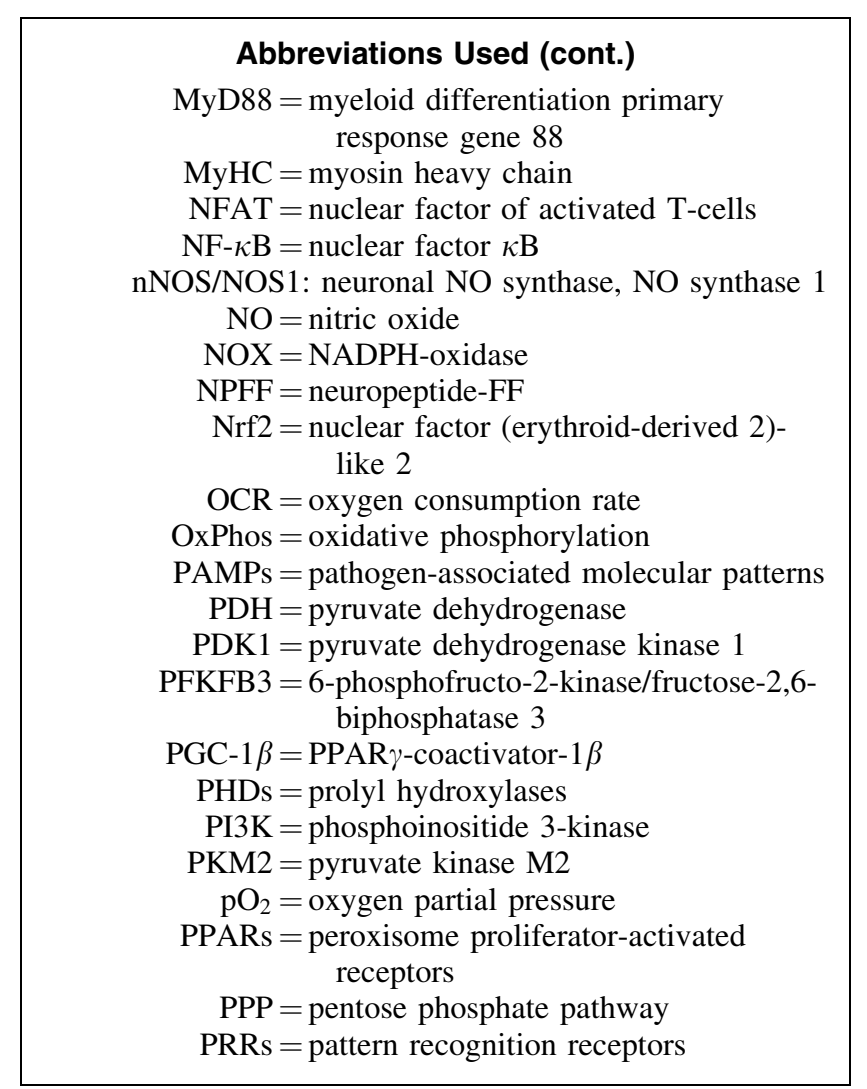

\begin{tabular}{|c|}
\hline 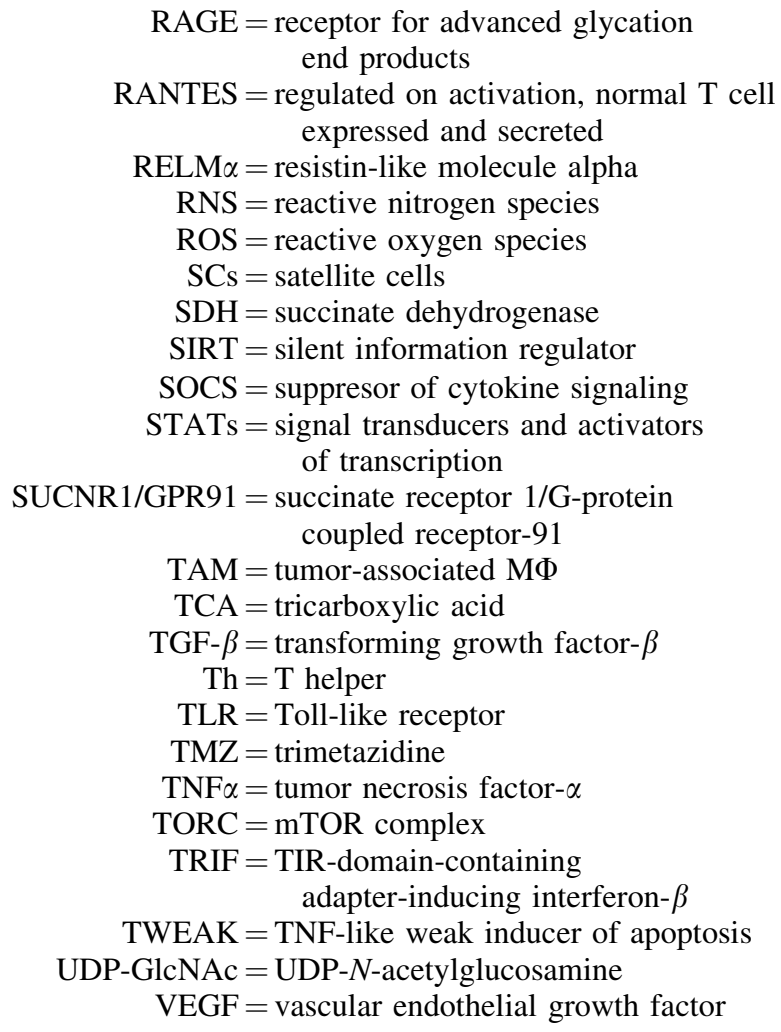 \\
\hline
\end{tabular}

